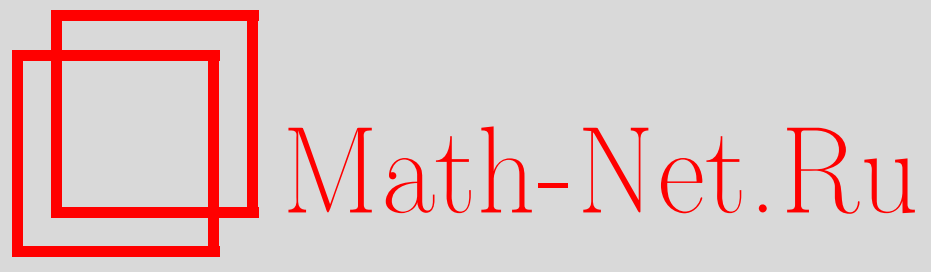

А. Л. Вольберг, В. Я. Эйдерман, Неоднородный гармонический анализ: 16 лет развития, УМН, 2013, том 68, выпуск 6, 3-58

DOI: https://doi.org/10.4213/rm9556

Использование Общероссийского математического портала Math-Net.Ru подразумевает, что вы прочитали и согласны с пользовательским соглашением http://www . mathnet.ru/rus/agreement

Параметры загрузки:

IP : 107.22 .136 .117

26 апреля 2023 г., 13:27:20

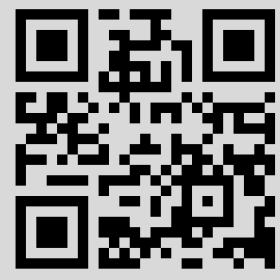




\title{
Неоднородный гармонический анализ: 16 лет развития
}

\begin{abstract}
А. Л. Вольберг, В. Я. Эйдерман
В обзоре излагаются результаты и методы теории сингулярных интегралов, получившей бурное развитие в течение последних 15-20 лет. Центральная (но не единственная) тема статьи - связь между аналитическими свойствами интегралов, а также операторов с ядрами Кальдерона-Зигмунда, и геометрическими свойствами меры. Прослеживается история классической задачи Пенлеве об описании устранимых особенностей ограниченных аналитических функций, явившейся мощным стимулом для развития этой ветви гармонического анализа. Прогресс последних десятилетий во многом основан на создании аппарата для работы с неоднородными мерами, которому в данной работе уделяется большое внимание. Формулируется ряд открытых вопросов, прежде всего в многомерном случае, в котором отсутствует метод кривизны меры.

Библиография: 128 названий.

Ключевые слова: аналитическая емкость, гипотеза Витушкина, операторы и емкости Кальдерона-Зигмунда, $T(1)$ - и $T(b)$-теоремы, спрямляемые множества и меры, сингулярные интегралы и операторы.
\end{abstract}

DOI: $10.4213 / \mathrm{rm} 9556$

\section{СоДЕРЖАНИЕ}

1. Введение................................................. 4

2. Задача Пенлеве и аналитическая емкость.................... 5

3. Ограниченность операторов с ядром Кальдерона-Зигмунда. T(1)- и

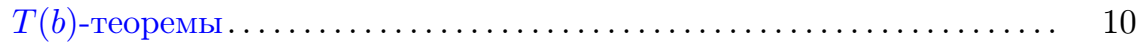

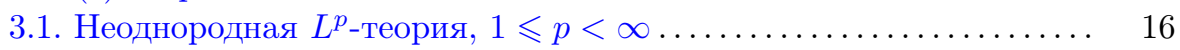

3.2. Более общие ядра и операторы Кальдерона-Зигмунда на мет-

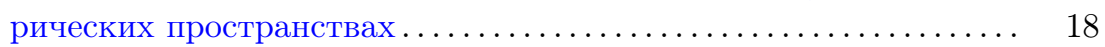

4. Емкости Кальдерона-Зигмунда ............................ 19

5. Оценки преобразования Рисса и емкостей. Емкость и мера Хаусдорфа 25

5.1. Оценка емкостей Кальдерона-Зигмунда. Связь с емкостями из нелинейной теории потенциала............................ 26

5.2. Емкости Кальдерона-Зигмунда и мера Хаусдорфа........... 28

5.3. Оценки картановского типа ....................... 30

Работа первого автора выполнена при поддержке Национального научного фонда США (грант DMS-0758552).

(C) А. Л. ВольБерг, В.Я. ЭйдЕрмАН, 2013 


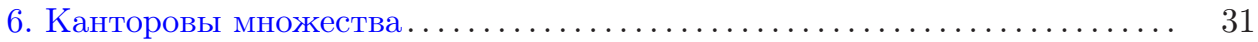

6.1. Емкость $\gamma_{s,+}$ канторовых множеств.................. 31

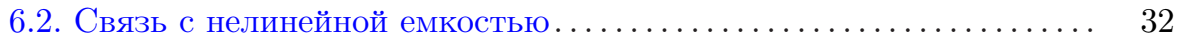

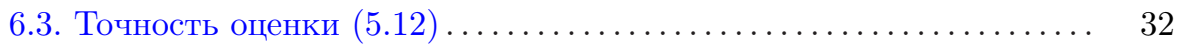

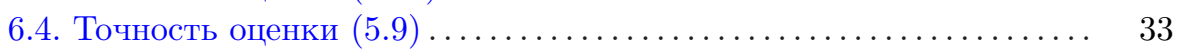

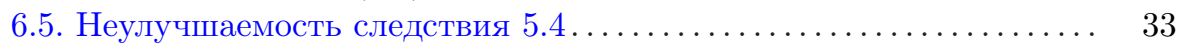

6.6. Длина Фавара канторовых множеств .................... 33

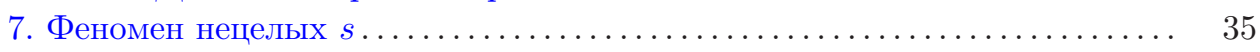

7.1. Ограниченность преобразований Рисса ................. 35

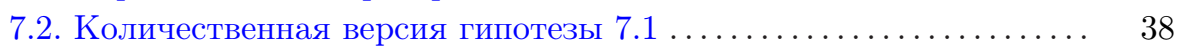

7.3. Существование главных значений и другие свойства преобразо-

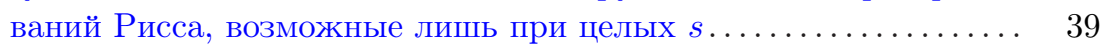

8. Существование главных значений в случае целых $s \ldots \ldots \ldots \ldots \ldots \ldots \ldots, 41$

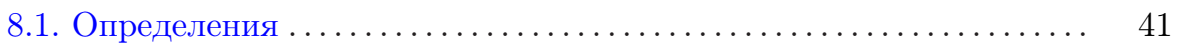

8.2. Существование главных значений и спрямляемость ......... 42

8.3. Существование главных значений преобразования $R^{n} \mu$ и огра-

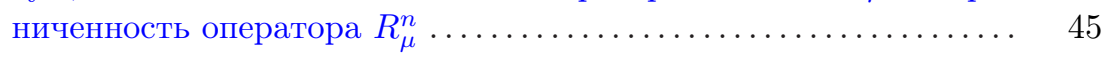

9. Ограниченность операторов Кальдерона-Зигмунда и спрямляемость.

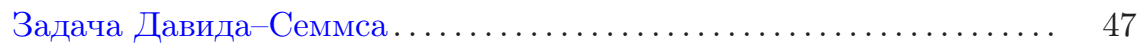

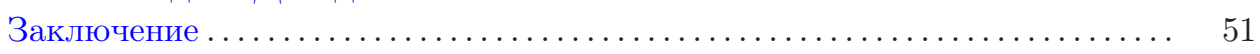

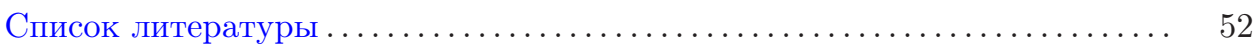

\section{1. Введение}

Тема данного обзора - некоторые результаты и тенденции развития теории сингулярных интегралов, появившиеся в течение последних 15-20 лет, а также открытые проблемы данной теории. Эта ветвь гармонического анализа тесно переплетена с теорией аналитических функций, теорией потенциала, геометрической теорией меры, теорией операторов (список далеко не полон). Так, исследования Кальдерона [11] были во многом вызваны потребностями теории дифференциальных уравнений с частными производными. Другим мощным стимулом для развития современной теории сингулярных интегралов явилась классическая задача Пенлеве - описать в метрических/геометрических терминах множества устранимых особенностей ограниченных аналитических функций. Ограниченность оператора Коши на графиках липшицевых функций с малой константой, установленная Кальдероном в 1977 г., оказалась ключевым фактом в доказательстве гипотезы Данжуа. Проблематика огромного количества последующих работ находится в русле этого результата. Ее можно очень приблизительно охарактеризовать следующим образом: какова связь между аналитическими свойствами сингулярных интегралов и операторов (например, ограниченностью векторных потенциалов Рисса и соответствующих операторов) и геометрическими свойствами меры (в частности, ее носителя)? Этот вопрос будет центральной (но не единственной) темой данной статьи.

Теория, развитая Зигмундом, Кальдероном, Давидом, Журне, Семмсом, Кристом и другими, относилась к пространствам однородного типа, снабженным мерой с условием удвоения $\mu(B(x, 2 r)) \leqslant C \mu(B(x, r)), x \in \operatorname{supp} \mu, r>0$. 
Давид получил ряд результатов без этого условия. Общая теория операторов Кальдерона-Зигмунда на пространствах неоднородного типа была построена Назаровым, Треилем и Вольбергом. Серия их работ начиная с 1997 г. (см. [86] отсюда число 16 в названии статьи) послужила основой и стимулом для дальнейшего бурного развития данной проблематики. Так, используя эти результаты, Толса [110] установил сравнимость аналитических емкостей $\gamma$ и $\gamma_{+}$и, как следствие, решил знаменитую задачу о полуаддитивности аналитической емкости. Важную роль в доказательстве сыграло понятие кривизны меры, введенное Мельниковым [79] в 1995 г. Этот инструмент "грубо отсутствует" ("cruelly missing" - известное выражение Ги Давида) для сингулярности ядра, большей единицы. Значение результатов Назарова, Треиля и Вольберга еще и в том, что понятие кривизны в них не используется. Идеи и методы, приведшие к "падению" условия удвоения, изложены в [122]. Некоторые из них будут рассмотрены ниже.

Перейдем к более подробному изложению.

\section{2. Задача Пенлеве и аналитическая емкость}

В 1947 г. Альфорс [3] ввел понятие аналитической емкости $\gamma(E)$ компакта $E \subset \mathbb{C}$ (сам термин "аналитическая емкость" был предложен Ерохиным в 1958 г.):

$$
\gamma(E)=\sup \left|f^{\prime}(\infty)\right|,
$$

где $f^{\prime}(\infty)=\lim _{z \rightarrow \infty} z(f(z)-f(\infty))$ и верхняя грань берется по всем функциям $f(z)$, голоморфным в $\mathbb{C} \backslash E$, для которых $|f(z)| \leqslant 1$ в $\mathbb{C} \backslash E$. Имеются и другие эквивалентные определения [40]. Для произвольных множеств емкость $\gamma$ определяется равенством

$$
\gamma(G)=\sup \{\gamma(E): E-\text { компакт, } E \subset G\} .
$$

Нетрудно показать - см., например, [3], [93], - что компакт E устраним для ограниченных аналитических функций тогда и только тогда, когда $\gamma(E)=0$. Устранимость означает, что для любого открытого множества $\Omega$, содержащего $E$, каждая ограниченная аналитическая функция в $\Omega \backslash E$ имеет аналитическое продолжение в $\Omega$. Разумеется, характеризация устранимых множеств в терминах аналитической емкости является лишь переформулировкой задачи Пенлеве, но не ее решением (об этом писал и сам Альфорс в [3]). По-настоящему "рабочими" аналитическую емкость и ее модификацию - непрерывную аналитическую емкость - сделал Витушкин [125], показав роль этих понятий в задачах рациональной аппроксимации.

Невозможно изложить в одной статье захватывающую историю задачи Пенлеве - см., например, монографии [40], [93], [30], [117], обзоры [80], [112], [113], [65], [66] - литература воистину огромна. Развитие теории Кальдерона-Зигмунда послужило основой замечательных успехов в решении этой классической задачи, а также других проблем теории аналитической емкости. Остановимся на некоторых из этих достижений.

Маттила, Мельников и Вердера решили задачу Пенлеве для регулярных компактов $E$ в $\mathbb{C}$ (т. е. в случае, когда длина множества $E \cap B(z, r)$ соизмерима 
с $r$ для любого круга $B(z, r), z \in E, 0<r \leqslant \operatorname{diam} E)-$ см. [70], где имеются ссылки на предшествующие работы. Пажо [92] вывел отсюда более общий результат, но при дополнительном условии о положительности нижней плотности меры $\mu=H^{1} \mid E$; здесь и далее $H^{s}$ означает $s$-мерную меру Хаусдорфа.

Давид [22] (см. также [27]) решил эту задачу для любых компактов конечной длины, т. е. при условии $H^{1}(E)<\infty$. Более краткое изложение идеи доказательства имеется в [23]. Несколько позднее эта задача была независимо (и проще) решена также Назаровым, Треилем и Вольбергом [89] (решение приведено и в [126]). Вероятностный подход, используемый в [89], облегчает доказательство следующей ниже теоремы 2.1. Также в [89] (и в [126]) доказана некоторая количественная версия теоремы 2.1 (неоднородная и слабо аккретивная версия $T(b)$-теоремы), которая оказалась крайне существенной при доказательстве Толсой [110] гипотезы Витушкина о полуаддитивности аналитической емкости (см. ниже).

Компакт Е называется совершенно неспрямляемым (purely unrectifiable), ecли $H^{1}(E \cap \Gamma)=0$ для любой спрямляемой кривой $\Gamma \subset \mathbb{C}$.

Теорема 2.1. Для компакта $E \subset \mathbb{C}$ конечной длины аналитическая емкость равна нулю тогда и толъко тогда, когда Е совершенно неспрямляем.

Точнее говоря, в [22] доказана только часть “£” теоремы 2.1 (совершенная неспрямляемость влечет равенство емкости нулю). Часть “”” вытекает из следующего классического утверждения.

Теорема 2.2. Пусть $E \subset \mathbb{C}$ - подмножество спрямляемой кривой. Тогда $\gamma(E)=0 \Longleftrightarrow H^{1}(E)=0$.

Часть “£" - теорема Пенлеве (1888), справедливая для любых компактов

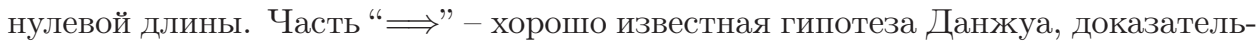
ство которой было завершено Кальдероном в 1977 г. Здесь существенно, что $E$ подмножество спрямляемой кривой. Первый пример компакта положительной длины и нулевой аналитической емкости был построен Витушкиным в 1959 г. Позднее Гарнетт и Иванов независимо показали, что таким свойством обладает "угловое" плоское канторово множество, при построении которого $n$-я генерация состоит из $4^{n}$ квадратов со стороной $l_{n}$, расположенных в углах квадратов предыдущей генерации, причем $l_{n}=l_{n-1} / 4, n=1,2, \ldots$. Указанный компакт является простейшим примером совершенно неспрямляемого множества положительной длины. Подробнее о канторовых множествах будет сказано в разделе 6 .

При некотором дополнительном предположении о регулярности компакта $E$ теорема 2.1 была доказана в [70] (см. раздел 7).

Теорему 2.1 можно сформулировать в терминах длины Фавара (называемой также вероятностью иглы Бюффона - Buffon needle probability):

$$
\operatorname{Fav}(E)=\frac{1}{\pi} \int_{0}^{\pi} H^{1}\left(P_{\theta}(E)\right) d \theta,
$$

где $P_{\theta}(E)$ - проекция компакта $E$ на прямую, проходящую под углом $\theta$ к действительной оси. В 1930-х годах Безикович показал, что компакт конечной 
длины совершенно неспрямляем тогда и только тогда, когда $\operatorname{Fav}(E)=0$ (см., например, [63], где имеется более общий результат). Таким образом, теорема 2.1 доказывает и знаменитую гипотезу Витушкина для компактов конечной длины: $\gamma(E)=0 \Longleftrightarrow \operatorname{Fav}(E)=0$.

Маттила [62] показал, что при $H^{1}(E)=\infty$ гипотеза Витушкина неверна. Но было неясно, имеет ли место хотя бы одна из импликаций, и если да, то какая. В [52] построен пример множества $E$ (разумеется, бесконечной длины), для которого $\gamma(E)>0$, но $\operatorname{Fav}(E)=0$. Вопрос о существовании множества с $\gamma(E)=0$ и $\operatorname{Fav}(E)>0$ до сих пор открыт; см. [93] для дополнительной информации. Связь между аналитической емкостью и длиной Фавара изучена далеко не полностью. Приведем результат Мюраи [82]: если $E$ - подмножество липшицевой кривой $\Gamma$, то $\gamma(E) \geqslant c \operatorname{Fav}(E)^{3 / 2} / \ell(\Gamma)^{1 / 2}$, где $\ell(\Gamma)$ - длина кривой $Г$. Мы вернемся к этому вопросу в п. 6.6.

Перейдем к теореме Толсы о сравнимости емкостей $\gamma$ и $\gamma_{+}$. Положительная аналитическая емкость $\gamma_{+}(E), E \subset \mathbb{C}$, определяется равенством

$$
\gamma_{+}(E)=\sup \mu(E)=\sup \|\mu\|,
$$

где супремум берется по положительным мерам, для которых $\operatorname{supp} \mu \subset E$, преобразование Коши

$$
\mathscr{C} \mu(z):=\int \frac{d \mu(\xi)}{\xi-z}
$$

принадлежит $L^{\infty}(\mathbb{C})$ и $\|\mathscr{C} \mu\|_{L^{\infty}(\mathbb{C})} \leqslant 1$. Поскольку интеграл в определении $\mathscr{C} \mu(z)$ сходится почти всюду относительно плоской меры Лебега, данное определение корректно. Из равенства $\left|(\mathscr{C} \mu)^{\prime}(\infty)\right|=\mu(E)$ сразу следует, что $\gamma_{+}(E) \leqslant \gamma(E)$. Замечательный результат Толсы [110] состоит в том, что $\gamma(E) \leqslant C \gamma_{+}(E)$ с абсолютной постоянной $C$. Идея доказательства объясняется в более кратких статьях [112], [113], [61]. Для канторовых множеств этот результат был ранее получен в [60]. Поскольку определение емкости $\gamma_{+}$аналогично определению классической ньютоновой емкости, соотношение $\gamma_{+} \approx \gamma$ оправдывает термин “емкость" применительно к $\gamma$. Теория потенциала для емкости $\gamma_{+}$развита в [109] и в [126] (см. также [110], [112], [113]). В частности, показано, что $\gamma_{+}$ счетно полуаддитивна. Исключительно важным следствием этого факта и соотношения $\gamma_{+} \approx \gamma$ является полуаддитивность, и даже счетная полуаддитивность, аналитической емкости:

$$
\gamma\left(\bigcup_{i} E_{i}\right) \leqslant C \sum_{i} \gamma\left(E_{i}\right),
$$

где $E_{i}, i=1,2, \ldots,-$ не более чем счетное семейство компактов. Вопрос о справедливости этого неравенства долгое время оставался открытым.

Среди основных средств, использованных при доказательстве описанных выше результатов, выделим неоднородные $T(1)$ - и $T(b)$-теоремы [86]-[90] (о которых пойдет речь в следующем разделе) и понятие кривизны меры, или менгеровской кривизны (Menger curvature), введенное Мельниковым [79] и блестяще примененное Толсой [109]-[113].

Менгеровская кривизна тройки точек $x, y, z \in \mathbb{C}-$ это величина $c(x, y, z)=$ $1 / R(x, y, z)$, где $R(x, y, z)$ - радиус окружности, проходящей через $x, y, z ;$ если 
точки лежат на одной прямой (в частности, если хотя бы две из них совпадают), то полагаем $c(x, y, z)=0$. Кривизной борелевской меры $\mu$ в $\mathbb{C}$ называется число

$$
c^{2}(\mu)=\iiint c(x, y, z)^{2} d \mu(x) d \mu(y) d \mu(z)=\iiint \frac{1}{R(x, y, z)^{2}} d \mu(x) d \mu(y) d \mu(z) .
$$

Одним из важнейших свойств кривизны меры является тесная связь этого понятия с $L^{2}$-нормой срезанного преобразования Коши, определяемого равенством

$$
\mathscr{C}_{\varepsilon} \mu(z):=\int_{|\xi-z|>\varepsilon} \frac{d \mu(\xi)}{\xi-z} .
$$

Обозначим через $\Sigma_{s}, 0<s \leqslant d$, класс положительных борелевских мер $\mu$ в $\mathbb{R}^{d}$, $d \geqslant 2$, удовлетворяющих условию

$$
\mu(B(x, r)) \leqslant r^{s} \quad \text { для любых } x \in \mathbb{R}^{d}, r>0,
$$

где $B(x, r):=\left\{y \in \mathbb{R}^{d}:|y-x|<r\right\}$. Меры $\mu \in \Sigma_{1}$ называются мерами линейного роста.

Имеет место соотношение, установленное Мельниковым и Вердерой [81]: для любой меры $\mu$ линейного роста в $\mathbb{C}$ справедливо равенство

$$
\left\|\mathscr{C}_{\varepsilon} \mu\right\|_{L^{2}(\mu)}^{2}=\frac{1}{6} c_{\varepsilon}^{2}(\mu)+O(\mu(\mathbb{C}))
$$

здесь $c_{\varepsilon}^{2}(\mu)$ - "срезанная" версия кривизны, определяемая аналогично $c^{2}(\mu)$ с той разницей, что тройные интегралы в $(2.1)$ берутся по множеству $\{(x, y, z) \in$ $\left.\mathbb{C}^{3}:|x-y|,|y-z|,|x-z|>\varepsilon\right\}$. Доказательство основано на чудесном равенстве, открытом Мельниковым: для любых трех точек $z_{1}, z_{2}, z_{3} \in \mathbb{C}$

$$
\sum \frac{1}{z_{\sigma(2)}-z_{\sigma(1)}} \frac{1}{\overline{z_{\sigma(3)}-z_{\sigma(1)}}}=\frac{1}{R\left(z_{1}, z_{2}, z_{3}\right)^{2}},
$$

где сумма берется по шести перестановкам $\sigma$ индексов $1,2,3$. Доказательство равенства (2.4) элементарно и доступно старшекласснику, но его значение трудно переоценить. Левая часть естественно возникает при оценке $L^{2}$-нормы преобразования Коши, и (2.4) легко приводит к (2.3) - см. [81], [112], [113] и многие другие работы, а также раздел 5 ниже. Формула (2.3) вкупе с $T(1)$-теоремами является мощным средством оценивания нормы $\left\|\mathscr{C}_{\mu, \varepsilon}\right\|_{L^{2}(\mu) \rightarrow L^{2}(\mu)}$ срезанного оператора Коши

$$
\mathscr{C}_{\mu, \varepsilon} f(z):=\int_{|\xi-z|>\varepsilon} \frac{f(\xi) d \mu(\xi)}{\xi-z} .
$$

Используя (2.3), Мельников и Вердера [81] нашли новое и короткое доказательство теоремы Кальдерона [10] об ограниченности оператора Коши на графиках липшицевых функций, развитой Койфманом, Макинтошем и Мейером [19]. Другие применения будут указаны ниже.

Мельников в [79] и Толса в своей диссертации (см., например, [93]) охарактеризовали емкость $\gamma_{+}$в терминах кривизны:

$$
c \sup \frac{\|\mu\|^{3 / 2}}{\left(\|\mu\|+c^{2}(\mu)\right)^{1 / 2}} \leqslant \gamma_{+}(E) \leqslant C \sup \frac{\|\mu\|^{3 / 2}}{\left(\|\mu\|+c^{2}(\mu)\right)^{1 / 2}},
$$


где $c, C$ - абсолютные положительные постоянные и верхняя грань берется по мерам $\mu \in \Sigma_{1}$ с носителями на $E$. В дальнейшем вместо двойного неравенства используем знак $\approx$. Имеются и другие родственные характеризации (см. [61] [109]). Приведем еще один важный результат такого типа [110], [113], [117]; он получит дальнейшее развитие в последующих разделах. Положим $\left|\mathscr{C}_{\mu}\right|=\sup _{\varepsilon>0}\left\|\mathscr{C}_{\mu, \varepsilon}\right\|_{L^{2}(\mu) \rightarrow L^{2}(\mu)}$

Tеорема 2.3. Для любого компакта $E \subset \mathbb{C}$

$$
\begin{aligned}
\gamma_{+}(E) & \approx \sup \left\{\mu(E): \operatorname{supp} \mu \subset E, \mu \in \Sigma_{1},\left\|\mathscr{C}_{\varepsilon} \mu\right\|_{L^{2}(\mu)}^{2} \leqslant \mu(E) \forall \varepsilon>0\right\} \\
& \approx \sup \left\{\mu(E): \operatorname{supp} \mu \subset E, \mu \in \Sigma_{1}, c^{2}(\mu) \leqslant \mu(E)\right\} \\
& \approx \sup \left\{\mu(E): \operatorname{supp} \mu \subset E, \mu \in \Sigma_{1},\left|\mathscr{C}_{\mu}\right| \leqslant 1\right\} .
\end{aligned}
$$

Поскольку $\left|\mathscr{C}_{\mu \mid G}\right| \leqslant\left|\mathscr{C}_{\mu}\right|$ для любого множества $G$, то функция множества, определяемая последней (третьей) верхней гранью в (2.5), счетно полуаддитивна (даже с константой 1). Отсюда следует счетная полуаддитивность емкости $\gamma_{+}$. Аналог последнего соотношения в высших размерностях практически одновременно и независимо рассматривался Назаровым, Треилем и Вольбергом [90], [126], причем книга [126] охватывает и случай $d=2$ - см. раздел 4.

Множество $E \subset \mathbb{C}$ называется 1-спрямляемым, если существует счетное семейство спрямляемых кривых $\Gamma_{i}$ таких, что $H^{1}\left(E \backslash \bigcup \Gamma_{i}\right)=0$. Несложно доказать (см. [93; с. 12]), что каждое множество в $\mathbb{C}$ конечной длины можно разбить на два непересекающихся подмножества, одно из которых совершенно неспрямляемо, а второе 1-спрямляемо.

Леже [54] установил следующее геометрическое свойство кривизны.

Теорема 2.4. Пусть $H^{1}$-измеримое множество $E \subset \mathbb{C}$ удовлетворяет условиям $H^{1}(E)<\infty$ и $c^{2}\left(H^{1} \mid E\right)<\infty$. Тогда Е является 1-спрямляемым.

В силу $(2.3)$ условие $c^{2}\left(H^{1} \mid E\right)<\infty$ можно заменить условием $L^{2}\left(H^{1} \mid E\right)$-ограниченности преобразования Коши. Теорема 2.4 играет важную роль в доказательстве гипотезы Витушкина. Она была установлена также Давидом, новое доказательство дано Толсой [111]; историю этой теоремы см. в [30; с. 154]. В разделе 9 мы вернемся к сюжету, связанному с гипотезой Витушкина и теоремой 2.4.

Интересно отметить, что при решении задачи Пенлеве - классической задачи теории аналитических функций - совсем не используются методы комплексного анализа.

В заключение данного раздела отметим, что С. Я. Хавинсоном был развит иной подход к теории аналитической емкости, основанный на соотношениях двойственности в теории экстремальных задач - см. [53], где имеется обширная библиография. В [53] изучаются также прямые аналоги емкости $\gamma_{+}$, в определении которых перебираются действительные и комплексные меры. 


\section{3. Ограниченность операторов с ядром Кальдерона-Зигмунда. $T(1)-$ и $T(b)$-теоремы}

Будем говорить, что функция $K(x, y),(x, y) \in \mathbb{R}^{d} \times \mathbb{R}^{d} \backslash\{(x, y): x=y\}$, является ядром Кальдерона-Зигмунда порядка $s, s \in(0, d]$, если

$$
|K(x, y)| \leqslant \frac{A}{|x-y|^{s}} \quad \text { и }\left|K\left(x^{\prime}, y\right)-K(x, y)\right|,\left|K\left(y, x^{\prime}\right)-K(y, x)\right| \leqslant A \frac{\left|x-x^{\prime}\right|^{\varepsilon}}{|x-y|^{s+\varepsilon}}
$$

для $\left|x-x^{\prime}\right| \leqslant|x-y| / 2$ и некоторых постоянных $A \geqslant 0$ и $\varepsilon \in(0,1]$.

Линейный оператор $T=T_{\mu}$, удовлетворяющий условию

$$
T f(x)=\int K(x, y) f(y) d \mu(y) \quad \forall x \notin \operatorname{supp} f,
$$

называется сингулярным интегральным оператором с ядром К3 $K$ (здесь $\mu$ совсем не обязана быть мерой Лебега). Заметим, что одно и то же ядро может быть ассоциировано с различными интегральными операторами. Например, оператор умножения на данную функцию или оператор дифференцирования являются интегральными операторами с ядром $K(x, y) \equiv 0$ (оба они дают 0 вне носителя $f$ ). Требуются усилия, чтобы по ядру разумно определить оператор. Мы не можем просто сказать, что $T f(x)=\int K(x, y) f(y) d \mu(y)$, так как для $\mu$-почти всех $x$ функции $K(x, \cdot), K(\cdot, x)$, вообще говоря, не являются интегрируемыми, даже локально, в окрестности $x$. Поэтому в общем случае мы не определяем $T f$ во всех точках носителя меры. В то же время предполагается, что каким-то образом функция $T f$ продолжена на supp $\mu$ с соблюдением условия (3.1) и условия линейности $T$.

Однако для антисимметричного ядра $(K(x, y)=-K(y, x))$ мы можем (формально) записать билинейную форму $T$ в следующем виде:

$$
\langle T f, g\rangle=\iint K(x, y) f(y) g(x) d \mu(x) d \mu(y)=-\iint K(x, y) f(x) g(y) d \mu(x) d \mu(y),
$$

и поэтому

$$
\langle T f, g\rangle=\frac{1}{2} \iint K(x, y)[f(y) g(x)-f(x) g(y)] d \mu(x) d \mu(y) .
$$

Но для липшицевых (или просто гладких) функций $f, g$ с компактным носителем последнее выражение прекрасно определено как абсолютно сходящийся интеграл, если $K$ есть ядро КЗ. Так что для антисимметричных ядер КЗ последнее выражение можно считать "каноническим" определением билинейной формы оператора с этим ядром. Ограниченность теперь легко формулировать в терминах соответствующей оценки этой билинейной формы на плотном множестве гладких функций.

Для ядер без свойства антисимметрии мы можем сказать, что оператор с ядром K3 ограничен в $L^{2}(\mu)$ (не определяя оператор!), если срезки $\bar{T}_{\varepsilon}$ оператора равномерно ограничены как операторы, действующие из $L^{2}(\mu)$ в $L^{2}(\mu)$; здесь

$$
\bar{T}_{\varepsilon} f(x)=\int_{y: \varepsilon<|x-y|<1 / \varepsilon} K(x, y) f(y) d \mu(y) .
$$


Ниже мы увидим (теорема 3.6), что ограниченность оператора с ядром К3 в $L^{2}(\mu)$ влечет ограниченность во всех пространствах $L^{p}(\mu), 1<p<\infty$.

Удобно определить также гладкие срезки. Пусть $\varphi-$ функция на $\mathbb{R}_{+}$такая, что $\varphi \in C^{\infty}, \varphi=0$ на $[0,1], \varphi=1$ на $[2, \infty)$. Рассмотрим $\psi_{\varepsilon}(x):=\varphi(x / \varepsilon)-\varphi(\varepsilon x)$. Определим

$$
\widetilde{T}_{\varepsilon} f(x)=\int \psi_{\varepsilon}(|x-y|) K(x, y) f(y) d \mu(y) .
$$

Недавно Лайю и Треиль [55] показали, что ограниченность билинейной формы

$$
\langle T f, g\rangle:=\iint K(x, y) f(y) g(x) d \mu(y) d \mu(x)
$$

на функциях с компактными и разделенными носителями влечет равномерную ограниченность этих гладких срезок (регуляризаций). Более того, для многих интересных операторов, в частности для преобразований Гильберта или Рисса, отсюда следует также равномерная ограниченность и обычных срезок. Таким образом, все разумные определения ограниченности оператора с ядром КЗ совпадают.

Будем говорить, что оператор $T$ слабо ограничен, если найдутся такие постоянные $\Lambda \geqslant 1, C<\infty$, что $\left|\left\langle T \chi_{Q}, \chi_{Q}\right\rangle\right| \leqslant C \mu(\Lambda Q)$ для любого куба $Q$; здесь $\chi_{Q}$ - индикатор куба $Q, \Lambda Q$ - куб, гомотетичный с коэффициентом $\Lambda$ кубу $Q$ относительно центра этого куба. Очевидно, что для антисимметричных ядер это условие выполнено. Как обычно, через $T^{*}$ обозначается оператор, сопряженный к $T$.

Теорему Давида-Журне, получившую имя $T(1)$-теоремы, удобно формулировать не так, как это было сделано авторами в [25], где было показано, что условия $T(1) \in \mathrm{BMO}, T^{*}(1) \in \mathrm{BMO}$ плюс слабая ограниченность оператора $T$ эквивалентны ограниченности оператора $\mathrm{K} 3$ в $L^{2}$, а по-другому, в виде следующей теоремы о тестировании.

Tеорема 3.1. Пусть $K(x, y), x, y \in \mathbb{R}^{d}$, - ядро K3, и пусть $m$ есть $d$-мерная мера Лебега. Оператор $T$ с ядром $K$ порядка $d$ ограничен в $L^{2}\left(\mathbb{R}^{d}, m\right)$ тогда и только тогда, когда для любого куба (либо шара) $Q$ в $\mathbb{R}^{d}$ выполнень следующие тестирующие условия:

$$
\begin{aligned}
\left\|T \chi_{Q}\right\|_{2} & \leqslant C\left\|\chi_{Q}\right\|_{2}, \\
\left\|T^{*} \chi_{Q}\right\|_{2} & \leqslant C\left\|\chi_{Q}\right\|_{2} .
\end{aligned}
$$

В такой формулировке условие слабой ограниченности выполнено автоматически, так как оно следует из (3.4). Заметим, что порядок ядра КЗ здесь $d$, и это в точности соответствует порядку меры Лебега в $\mathbb{R}^{d}:|B(x, r)| \approx r^{d}$. Теорема 3.1 была распространена Кристом на операторы в метрических пространствах с мерой. Точная формулировка требует пространства $(X, \rho, \mu)$, где $(X, \rho)$ - метрическое пространство однородного типа (любой шар радиуса $r$ можно покрыть универсальным количеством $N$ шаров радиуса $r / 2)$, а $\mu$ - мера с условием удвоения. К слову: ясно, что метрическое пространство, на котором есть мера $\mu$ с условием удвоения такая, что $X=\operatorname{supp} \mu$, автоматически 
является пространством однородного типа. Это почти тривиально. Но верно и обратное. Вольберг и Конягин [127] доказали, что любое компактное метрическое пространство однородного типа несет меру с условием удвоения, для которой $X=\operatorname{supp} \mu$.

Таким образом, если $E$ есть локально компактное подмножество $\mathbb{R}^{d}$ с евклидовой метрикой и если мера $\mu$ удовлетворяет условию $\forall x \in E, \forall r \in(0, \operatorname{diam} E]$, $\mu(B(x, r)) \approx r^{s}, 0<s \leqslant d$, мы знаем, какие операторы с ядрами КЗ порядка $s$ ограничены в $L^{2}(E, \mu)$. По теореме Криста, это в точности операторы, удовлетворяющие аналогам условий $T(1)$-теоремы 3.1 с мерой и ядром порядка $s$ вместо $d$.

Однако доказательство теоремы 3.1 и ее обобщений существенно использовало, что порядок роста меры и порядок ядра одинаковы. Тем самым, остается "маленький" вопрос: что если мы имеем оператор с ядром КЗ порядка $s<d$ на $E$, а мера на $E$ не имеет никакой оценки? Например, $E$ - компакт на плоскости $(d=2)$ с мерой $\mu=H^{1} \mid E$ (таким образом, предполагается, что $\left.0<H^{1}(E)<\infty\right)$, а $K(x, y)=1 /(x+i y)=1 / z$.

Не очень трудно доказать (см. [21; предложение 1.4 , с. 56] или [86]), что ограниченность оператора с ядром порядка $s$ во всех "хороших" случаях (классические ядра, включая $1 / z$; для него, конечно, $s=1$ ) автоматически влечет неравенство $\mu(B(x, r)) \leqslant C r^{s}$. Так что оценку сверху на рост меры мы можем считать данной.

Однако остается следующий вопрос. Пусть мера имеет рост не выше $C r^{s}$, но не имеет никакой оценки снизу. Как узнать, что оператор с ядром порядка $s$ ограничен в $L^{2}(E, \mu)$ ?

Назаров, Треиль и Вольберг в [86] доказали следующую "неоднородную" $T(1)$-теорему.

Теорема 3.2. Пусть $K(x, y), x, y \in \mathbb{R}^{d}$, - ядро KЗ порядка $s$, и пусть мера $\mu$ удовлетворяет условию $\mu(B(x, r)) \leqslant C r^{s}$. Оператор $T$ с ядром $K$ ограничен в $L^{2}(\mu)$ тогда и только тогда, когда для каждого куба $Q$ выполнены следующие тестирующие условия:

$$
\begin{aligned}
\left\|T \chi_{Q}\right\|_{L^{2}(\mu)} & \leqslant C\left\|\chi_{Q}\right\|_{L^{2}(\mu)}, \\
\left\|T^{*} \chi_{Q}\right\|_{L^{2}(\mu)} & \leqslant C\left\|\chi_{Q}\right\|_{L^{2}(\mu)} .
\end{aligned}
$$

Мы набросаем сейчас схему доказательства, объяснив, в чем же была главная трудность, связанная с неоднородностью меры, и как она была преодолена. Главная идея здесь вероятностная.

Напомним подход к доказательству теоремы 3.1 и к доказательству ее обобщения, полученного Кристом. Идея: рассмотреть билинейную форму $\left\langle T_{\mu} f, g\right\rangle$ и

1) разложить функции $f$ и $g$ по базису Хаара (или по другому базису "волнушек") относительно диадической решетки $\mathscr{D}$;

$2)$ представить $\left\langle T_{\mu} f, g\right\rangle$ в виде двойной суммы $\sum_{Q, R \in \mathscr{D}}\left\langle T_{\mu} \Delta_{Q}^{\mu} f, \Delta_{R}^{\mu} g\right\rangle$, где $\Delta_{Q}^{\mu} f$, $\Delta_{R}^{\mu} g$ - проекции функций $f, g$ на хааровские подпространства, ассоциированные с кубами $Q, R$ соответственно; 
3) не спешить внести знак абсолютной величины внутрь суммы, а сначала разбить сумму на подсуммы $\Sigma_{\text {out }}, \Sigma_{\text {in }}, \Sigma_{\text {close }}$, имеющие следующий геометрический смысл: первая сумма имеет дело с парами $Q, R$ такими, что $\ell(Q)$, $\ell(R) \geqslant \operatorname{dist}(Q, R)$ (“дальнодействие"), вторая - конечно же, с парами, для которых $Q \subset R$ или $R \subset Q$, и, наконец, третья - со всеми остальными парами;

4) в $\Sigma_{\text {оut }}$ можно внести знак модуля внутрь суммы (эта сумма, отвечающая за "дальнодействие", самая простая), а в других двух суммах надо аккуратно группировать члены огромными кусками; мы не будем на этом останавливаться.

Но вот что важно: для каждой из трех сумм применяется свой метод оценки членов вида $\left|\int_{Q} \int_{R} K(x, y) f_{Q}(x) g_{R}(y) d \mu(x) d \mu(y)\right|$ (где $f_{Q}, g_{R}$ суть $\Delta_{Q}^{\mu} f, \Delta_{R}^{\mu} g$ ). Для оценивания такого билинейного выражения естественно использовать один из следующих трех приемов.

а) Оценка через нормы $\|K\|_{L^{\infty}(\mu|Q \times \mu| R)},\left\|f_{Q}\right\|_{L^{1}(\mu)},\left\|g_{R}\right\|_{L^{1}(\mu)}$ - этот метод применяется для первой суммы ("дальнодействия"), и с ним нет проблем, так как существенная часть оценки будет иметь вид $\sqrt{\mu(Q) \mu(R)}$ (мы используем очевидное неравенство $\left.\left\|f_{Q}\right\|_{L^{1}(\mu)} \leqslant\left\|f_{Q}\right\|_{L^{2}(\mu)} \sqrt{\mu(Q)}\right)$.

b) Оценка через нормы $\|K\|_{L^{1}(\mu) L^{\infty}(\mu)}:=\left\|\int_{Q}|K(x, y)| d \mu(x)\right\|_{L^{\infty}(\mu)},\left\|f_{Q}\right\|_{L^{\infty}(\mu)}$, $\left\|g_{R}\right\|_{L^{1}(\mu)}$ (или симметричный случай $\left.\|K\|_{L^{\infty}(\mu) L^{1}(\mu)},\left\|f_{Q}\right\|_{L^{1}(\mu)},\left\|g_{R}\right\|_{L^{\infty}(\mu)}\right)-$ этот тип оценки применяется при $R \subset Q$ (тут мы используем то обстоятельство, что функция $f_{Q}$ почти постоянна на $Q$, что означает выполнение неравенства $\left.\left\|f_{Q}\right\|_{L^{\infty}(\mu)} \leqslant C\left\|f_{Q}\right\|_{L^{2}(\mu)} / \sqrt{\mu(Q)}\right)$. Теперь существенная часть оценки будет иметь вид $\sqrt{\mu(R) / \mu(Q)}$.

с) Оценка через $\|K\|_{L^{1}(\mu|Q \times \mu| R)},\left\|f_{Q}\right\|_{L^{\infty}(\mu)},\left\|g_{R}\right\|_{L^{\infty}(\mu)}$, что приводит к оценке с существенной частью $1 / \sqrt{\mu(R) \mu(Q)}$.

Последняя оценка в нашем случае бесполезна: мера может быть сколь угодно мала, нет никакой оценки снизу и малые знаменатели "взрывают" любую оценку. Значит, можно пользоваться только оценками вида а), b).

Но оценка b) хороша исключительно для $R \subset Q$ (а симметричная ей оценка - только для $Q \subset R$ ). Действительно, попытаемся применить b) для куба $R$, расположенного вблизи, но не внутри $Q$ (пусть даже "очень маленького" по сравнению с размером $Q)$. Поскольку мера $\mu(Q)$ может быть сколь угодно малой по сравнению с $\mu(R)$, выражение $\sqrt{\mu(R) / \mu(Q)}$ опять может иметь "малый" знаменатель и неконтролируемо в принципе. Значит, остается использовать оценку а) для близких кубов $R, Q$. Но это тоже не годится, так как $L^{\infty}$-норма ядра будет "взрываться".

Выхода, казалось бы, нет. Описанный выше метод доказательства главной теоремы теории сингулярных интегралов K3 очевидно не может быть распространен на меры с плохим поведением - мы описали "непреодолимое" препятствие. И никакого другого метода не было.

Обойти указанное выше "непреодолимое" препятствие можно, используя "случайные" геометрические построения. Опишем одно из них (подробное изложение имеется в [86], [90]). Вместо одной фиксированной диадической решетки 
возьмем две, причем случайные, независимые решетки $\mathscr{D}_{1}, \mathscr{D}_{2}$. С множеством пар таких решеток нетрудно ассоциировать вероятностное пространство пар $\left(\omega_{1}, \omega_{2}\right) \in Q_{0}^{2}$ с мерой Лебега в качестве вероятности Р, где $Q_{0}$ - единичный куб B $\mathbb{R}^{d}$.

Теперь введем понятие $r$-плохого куба. Куб $R$ решетки $\mathscr{D}_{2}$ называется $r$-плохим, если найдется куб $Q$ решетки $\mathscr{D}_{1}$, который по меньшей мере в $2^{r}$ раз больше по размеру нашего куба $R$ и для которого $\ell(R)^{\gamma} \ell(Q)^{1-\gamma} \geqslant \operatorname{dist}(\partial R, \partial Q)$; здесь $\gamma=\varepsilon /(2(d+\varepsilon))$ и $\varepsilon-$ константа из определения ядра КЗ $K$. Легко видеть, что плохими являются те кубы $R$, которые находятся близко к границе какого-нибудь большого куба $Q$ и при этом не очень малы. Нетрудно оценить вероятность куба быть $r$-плохим: $\mathrm{P}\left(R \in \mathscr{D}_{2}, R\right.$ является $r$-плохим $) \leqslant 2^{-c r}$, где $c>0$ зависит только от $d$ и $\gamma$. Заметим, что эта вероятность не зависит от куба. Замена $\mathscr{D}_{2}$ на $\mathscr{D}_{1}$ полностью симметрична. Теперь зафиксируем большое число $r$ и разобьем неслучайные функции $f, g$ на случайные слагаемые: $f=f_{g}+f_{b}, g=g_{g}+g_{b}$, где $f_{b}:=\sum_{Q \text { плохой }} \Delta_{Q} f$ и функция $g_{b}$ определяется
аналогично.

Замечательное обстоятельство заключается в том, что математическое ожидание случайных плохих кусков $f_{b}, g_{b}$ мало:

$$
\mathrm{E}\left\|f_{b}\right\|_{L^{2}(\mu)}^{2}=\mathrm{E}_{\omega_{1}} \sum_{Q \in \mathscr{D}_{1}}\left\|\Delta_{Q} f\right\|_{L^{2}(\mu)}^{2} \mathrm{P}_{\omega_{2}}(Q \text { плохой }) \leqslant 2^{-c r}\|f\|_{L^{2}(\mu)}^{2} .
$$

Теперь, приближая $T$ заведомо ограниченными операторами $T_{\delta}$ (срезками), мы можем "вытащить себя за волосы" с помощью самооценки

$$
\left|\left\langle T_{\delta} f, g\right\rangle\right|=\left|\mathrm{E}\left[\left\langle T_{\delta} f_{g}, g_{g}\right\rangle+\left\langle T_{\delta} f_{g}, g_{b}\right\rangle+\left\langle T_{\delta} f_{b}, g_{g}\right\rangle+\left\langle T_{\delta} f_{b}, g_{b}\right\rangle\right]\right| \cdot
$$

Согласно только что сказанному, математическое ожидание каждого из последних трех слагаемых ограничено сверху величиной $2^{-c r}\left\|T_{\delta}\right\|\|f\|_{L^{2}(\mu)}\|g\|_{L^{2}(\mu)}$. Выберем функции $f, g$ такими, что $\|f\|_{L^{2}(\mu)}=\|g\|_{L^{2}(\mu)}=1$ и $\left|\left\langle T_{\delta} f, g\right\rangle\right| \geqslant\left\|T_{\delta}\right\| / 2$. Мы сразу получим оценку $\left\|T_{\delta}\right\| / 2 \leqslant\left|\mathrm{E}\left\langle T_{\delta} f_{g}, g_{g}\right\rangle\right|+3 \cdot 2^{-c r} \cdot\left\|T_{\delta}\right\|$. Выбирая $r$ достаточно большим, приходим к неравенству $\left\|T_{\delta}\right\| / 4 \leqslant\left|\mathrm{E}\left\langle T_{\delta} f_{g}, g_{g}\right\rangle\right|$. Но все пары кубов в последнем математическом ожидании могут быть обслужены нашими оценками a), b) (мы объясним это ниже). Поэтому можно просто повторить классическое доказательство теоремы 3.1 - ведь все опасные члены с малыми знаменателями просто не появляются в $\left\langle T_{\delta} f_{g}, g_{g}\right\rangle$ и этот член оценивается через параметры $T(1)$-теоремы без всяких изменений.

Как же исключаются члены с малыми знаменателями? Члены из $\Sigma_{\text {out }}$ (дальнодействие) в $\left\langle T_{\delta} f_{g}, g_{g}\right\rangle$, как всегда, имеют хорошую оценку $\sqrt{\mu(R) \mu(Q)}$. Члены $\Sigma_{\text {in }}$ в сумме $\left\langle T_{\delta} f_{g}, g_{g}\right\rangle$ уже были более или менее хороши, так как оцениваются через $\sqrt{\mu(R) / \mu(Q)}$, где $R \subset Q$ (тут надо быть аккуратным, поскольку таких членов очень много, но мы опускаем детали). Однако оценки типа b),

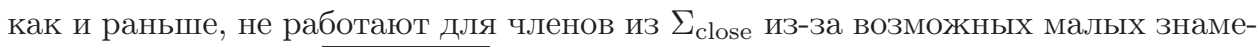
нателей в дробях $\sqrt{\mu(R) / \mu(Q)}$ с $R$, не лежащими внутри $Q$. Новизна ситуации состоит в том, что теперь в сумме $\left\langle T_{\delta} f_{g}, g_{g}\right\rangle$ встречаются только хорошие кубы. Следовательно, возможны лишь два случая. 
(1) Два куба $(Q, R)$ из $\Sigma_{\text {close }}$ не только близки в евклидовой метрике, но и $r$-близки в диадическом смысле (т.е. их размеры сравнимы). Такие пары оцениваются с помощью тест-условий доказываемой теоремы.

(2) Расстояние между $Q$ и $R$ во много раз больше размера меньшего из них. Тогда оценка проводится точно так же, как и в случае а) для удаленных кубов, и не требует даже тест-условий; используются лишь свойства ядра КЗ.

Таким образом, из неравенства $\left\|T_{\delta}\right\| \leqslant 4\left|\mathrm{E}\left\langle T_{\delta} f_{g}, g_{g}\right\rangle\right|$ мы получаем равномерную по $\delta$ оценку нормы $\left\|T_{\delta}\right\|$ через константы тест-условий и ядра КЗ. Это и означает, что мы оценили норму оператора $T$. Так доказывается неоднородная T(1)-теорема в работе [86].

Для нужд теории аналитической емкости (и других емкостей КЗ) необходимы более гибкие и трудные $T(b)$-теоремы. Назовем ограниченную функцию $b$ слабо аккретивной (относительно меры $\mu$ ), если для некоторой постоянной $\delta>0$ выполнено условие

$$
\frac{1}{\mu(Q)}\left|\int_{Q} b d \mu\right| \geqslant \delta>0 \quad \text { для любого куба } Q .
$$

Давид, Журне и Семмс в работе [26] доказали следующий аналог теоремы 3.1 ( $T(b)$-теорему). Мы формулируем ее опять по-своему, как тест-теорему.

Tеорема 3.3. Пусть $T$ - оператор с ядром $K 3$ К порядка $d$ в $\mathbb{R}^{d}$, и пусть $m$ есть $d$-мерная мера Лебега. Оператор $T$ ограничен в $L^{2}\left(\mathbb{R}^{d}, m\right)$ тогда и только тогда, когда для слабо аккретивной функции $b$, некоторой константы $C$ и каждого куба $Q$ выполняются следующие условия:

$$
\begin{aligned}
\left\|T\left(b \chi_{Q}\right)\right\|_{2} & \leqslant C\left\|b \chi_{Q}\right\|_{2}, \\
\left\|T^{*}\left(b \chi_{Q}\right)\right\|_{2} & \leqslant C\left\|b \chi_{Q}\right\|_{2} .
\end{aligned}
$$

Известно много переформулировок и вариантов этой теоремы. Имеется однородная $T(b)$-теорема Криста [18] (мера Лебега $m$ становится какой-нибудь мерой с условием удвоения), ему же принадлежит локальная однородная версия, когда функции $b$ (но не константе $\delta$ ) разрешено зависеть от $Q$. При этом в условиях (3.9), (3.10) можно иметь разные слабо аккретивные функции. Более того, очень полезной оказалась и такая версия, когда мы меняем (3.9) на $T^{\sharp}(b) \in L^{\infty}(\mu)$, а $(3.10)$ на $\left(T^{*}\right)^{\sharp}(b) \in L^{\infty}(\mu)$; определение максимального оператора $T^{\sharp}$ приводится ниже (см. формулу (3.11)).

Назаров, Треиль и Вольберг [90] доказали эту теорему для неоднородных мер без условия удвоения. Более того, в правых частях (3.9), (3.10) можно поставить бо́льшую величину $\left\|b \chi_{\Lambda Q}\right\|$ с любым числом $\Lambda>1$. Еще более важной оказалась следующая версия теоремы: можно потребовать выполнение условия (3.8) лишь на одном кубе $Q$ ! Очевидно, что в этом случае оператор, вообще говоря, уже не будет ограниченным в $L^{2}(\mu)$. Но имеется подмножество $E^{\prime}$, $\mu\left(E^{\prime}\right)>0$, такое, что оператор ограничен в $L^{2}\left(E^{\prime}, \mu\right)$. Эта $T(b)$-теорема доказана в [89]. Она послужила краеугольным камнем в доказательстве гипотезы Витушкина [89] и в решении Толсой задачи о полуаддитивности аналитической емкости [110], а также была использована в [126]. В доказательстве опять применяется геометрическая конструкция наподобие описанной выше, но теперь и 
операторы $T_{\delta}$ становятся случайными. Это очень тонкий, трудный, но очень полезный результат.

Техника Назарова-Треиля-Вольберга развивалась, в частности, в работе [47], в которой ослаблены тестирующие условия, и в статье [46], где рассмотрены также более общие условия на меру, описанные в следующем п. 3.1.

3.1. Неоднородная $L^{p}$-теория, $1 \leqslant p<\infty$. Здесь мы изложим более общий подход к теории КЗ. Зафиксируем $s>0$ (не обязательно целое). Пусть $\mathscr{X}$ - сепарабельное метрическое пространство, снабженное положительной мерой $\mu$ размерности $s$, т. е. удовлетворяющей условию

$$
\mu(B(x, r)) \leqslant r^{s} \quad \text { для любых } x \in \mathscr{X}, r>0 .
$$

Читатель может думать, что $\mathscr{X}$ - это $\operatorname{supp} \mu$, где $\mu$ - мера в евклидовом пространстве $\mathbb{R}^{d}$ с указанным выше условием роста (т. е. из класса $\Sigma_{s}$ ), и метрика на $\mathscr{X}$ индуцирована обычной евклидовой метрикой.

Пусть $L^{p}(\mu), 1 \leqslant p \leqslant \infty,-$ обычное пространство Лебега. Определим пространство $L^{1, \infty}(\mu)$ как множество таких функций $f: \mathscr{X} \rightarrow \mathbb{C}$, для которых

$$
\|f\|_{L^{1, \infty}(\mu)}:=\sup _{t>0} t \cdot \mu\{x \in \mathscr{X}:|f(x)|>t\}<+\infty .
$$

Заметим, что "норма" $\|f\|_{L^{1, \infty}(\mu)}$ не есть настоящая норма в том смысле, что она не удовлетворяет неравенству треугольника. Однако

$$
\|c f\|_{L^{1, \infty}(\mu)}=|c| \cdot\|f\|_{L^{1, \infty}(\mu)} \quad \text { и } \quad\|f+g\|_{L^{1, \infty}(\mu)} \leqslant 2\left(\|f\|_{L^{1, \infty}(\mu)}+\|g\|_{L^{1, \infty}(\mu)}\right)
$$

для $c \in \mathbb{C}$ и $f, g \in L^{1, \infty}(\mu)$.

Пусть $M(\mathscr{X})$ - множество комплексных мер на $\mathscr{X}$. Как всегда, $\|\nu\|$ означает полную вариацию меры $\nu \in M(\mathscr{X})$.

Классическое ядро КЗ порядка (размерности) $s$ на $\mathscr{X}$ определяется так же, как и в случае $\mathbb{R}^{d}$. А именно, это функция $K(x, y): \mathscr{X} \times \mathscr{X} \rightarrow \mathbb{C}, x \neq y$, которая при некоторых постоянных $A>0$ и $\varepsilon \in(0,1]$ удовлетворяет неравенствам

(1) $|K(x, y)| \leqslant \frac{A}{\operatorname{dist}(x, y)^{s}}$,

(2) $\left|K(x, y)-K\left(x^{\prime}, y\right)\right|,\left|K(y, x)-K\left(y, x^{\prime}\right)\right| \leqslant A \cdot \frac{\operatorname{dist}\left(x, x^{\prime}\right)^{\varepsilon}}{\operatorname{dist}(x, y)^{s+\varepsilon}}$,

как только $x, x^{\prime}, y \in \mathscr{X}$ и $\operatorname{dist}\left(x, x^{\prime}\right) \leqslant \operatorname{dist}(x, y) / 2$.

ЗАмечАниЕ 3.4. Хотя мы постоянно называем число $s$ "размерностью", но, как правило, это не есть размерность пространства $\mathscr{X}$ ни в каком смысле. Это размерность меры $\mu$ или ядра $K$, но никак не $\mathscr{X}$. Например, для ядра Коши на плоскости $s$ равно 1 , а не 2! Более того, топологическая размерность пространства $\mathscr{X}$ может быть даже бесконечной.

ОПРЕДЕЛЕНИЕ 3.5. Ограниченный в $L^{2}(\mu):=L^{2}(\mathscr{X}, \mu)$ сингулярный интегральный оператор $T$ с ядром КЗ, понимаемый в том же смысле, что и в случае $\mathbb{R}^{d}$, называется оператором Кальдерона-Зигмунда.

Очевидно, что сопряженный к оператору КЗ оператор $T^{*}$ также ограничен в $L^{2}(\mu)$ и также имеет ядро $\mathrm{K} 3 K^{*}(x, y)=\overline{K(y, x)}$. 
Для меры $\nu \in M(\mathscr{X})$ полагаем

$$
T \nu(x):=\int_{\mathscr{X}} K(x, y) d \nu(y), \quad x \in \mathscr{X} \backslash \operatorname{supp} \nu .
$$

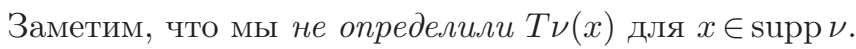

Максимальный сингулярный оператор $T^{\sharp}$, ассоциированный с оператором KЗ T, определяется равенствами

$$
\begin{aligned}
& T^{\sharp} f(x):=\sup _{r>0}\left|T_{r} f(x)\right|, \quad f \in L^{p}(\nu), \\
& \text { где } T_{r} f(x):= \int_{\mathscr{X} \backslash B(x, r)} K(x, y) f(y) d \nu(y), \\
& T^{\sharp} \nu(x):=\sup _{r>0}\left|T_{r} \nu(x)\right|, \quad \nu \in M(\mathscr{X}), \\
& \text { где } T_{r} \nu(x):=\int_{\mathscr{X} \backslash B(x, r)} K(x, y) d \nu(y) .
\end{aligned}
$$

Следующий результат звучит как классическая теорема Кальдерона-Зигмунда 1950-х годов [12]. Но эта неоднородная теорема была доказана в 1998 г. в статье [87].

ТеОРема 3.6. Для любого оператора КЗ $T$ (ограниченного в $\left.L^{2}(\mu)\right)$ выполнены следующие утверждения.

(1) $L^{p}$-действие. Для каждого $p \in(1,+\infty)$ оператор $T$ ограничен в $L^{p}(\mu)$ в том смысле, что

$$
\|T f\|_{L^{p}(\mu)} \leqslant C\|f\|_{L^{p}(\mu)}
$$

для любой функиии $f \in L^{p}(\mu) \cap L^{2}(\mu)$, где $C>0$ не зависит от $f$.

(2) Слабый тип. Oператор $T$ из $L^{1}(\mu)$ в $L^{1, \infty}(\mu)$ ограничен в том смысле, что

$$
\|T f\|_{L^{1, \infty}(\mu)} \leqslant C\|f\|_{L^{1}(\mu)}
$$

для любой функиии $f \in L^{1}(\mu) \cap L^{2}(\mu)$, где $C>0$ не зависит от $f$.

(3) Максимальный сингулярный оператор на $L^{p}(\mu)$. Для $p \in(1,+\infty)$ оператор $T^{\sharp}$ ограничен в $L^{p}(\mu)$ в том смысле, что

$$
\left\|T^{\sharp} f\right\|_{L^{p}(\mu)} \leqslant C\|f\|_{L^{p}(\mu)}
$$

для любой функции $f \in L^{p}(\mu)$, где $C>0$ не зависит от $f$.

(4) Слабый тип максимального сингулярного оператора. Onepamop $T^{\sharp} u з$ $M(\mathscr{X})$ в $L^{1, \infty}(\mu)$ ограничен в том смысле, что

$$
\left\|T^{\sharp} \nu\right\|_{L^{1, \infty}(\mu)} \leqslant C\|\nu\|
$$

для любой меры $\nu \in M(\mathscr{X})$, где $C>0$ не зависит от $\nu$.

ЗАмечАниЕ 3.7. Теорема остается в силе, если заменить условие ограниченности оператора $T$ в $L^{2}(\mu)$ условием ограниченности в $L^{p_{0}}(\mu), 1<p_{0}<\infty$. Возможно (хотя и значительно сложнее) доказать, что слабая ограниченность 
при $p_{0}=1$ (слабый тип) $\|T\|_{L^{1}(\mu) \rightarrow L^{1, \infty}(\mu)}<+\infty$ также достаточна. Имеются примеры (см. [85], [99]), когда много легче проверить слабый тип, чем $L^{2}(\mu)$-ограниченность оператора с ядром K3.

О доказательстве: в случае операторов $\mathrm{K} 3$ в $\mathbb{R}^{d}$ с мерой Лебега теорема 3.6 доказана Кальдероном и Зигмундом в фундаментальной работе [12]. Метод очень красив и стал одним из стандартных методов многих теорий; он называется методом разложения КЗ. Этот метод без изменения подходит для мер $\mu$ с условием удвоения. Но для наших мер без условия регулярности он не может быть применен непосредственно. Поэтому в [87] этот метод не используется. К цели приводит другое комбинаторное рассуждение.

Однако Толса [108] заметил, что идеи разложения КЗ все же можно использовать и для плохих мер.

3.2. Более общие ядра и операторы Кальдерона-Зигмунда на метрических пространствах. В ряде ситуаций удобно иметь более общее определение ядер КЗ на метрическом пространстве $\mathscr{X}$. Такие ядра были введены Хютоненом и Мартикайненом в [45].

Пусть $\mathscr{X}$ - метрическое пространство со следующим геометрическим условием удвоения: каждый открытый шар $B(x, r)$ можно покрыть не более чем $N$ шарами радиуса $r / 2$, причем конечное число $N$ не зависит от $x, r$. Пусть, далее, $\lambda(x, r)$ - положительная функция, возрастающая по $r$ и такая, что $\lambda(x, 2 r) \leqslant$ $C \lambda(x, r)$, где постоянная $C$ не зависит от $x$ и $r$.

Ядро Кальдерона-Зигмунда, ассоциированное с функцией $\lambda$, вводится аналогично предыдущим случаям: это функция $K(x, y): \mathscr{X} \times \mathscr{X} \rightarrow \mathbb{C}, x \neq y$, удовлетворяющая условиям

$$
\begin{gathered}
|K(x, y)| \leqslant C \min \left(\frac{1}{\lambda(x, \operatorname{dist}(x, y))}, \frac{1}{\lambda(y, \operatorname{dist}(x, y))}\right), \\
\left|K(x, y)-K\left(x^{\prime}, y\right)\right| \leqslant C \frac{\operatorname{dist}\left(x, x^{\prime}\right)^{\varepsilon}}{\operatorname{dist}(x, y)^{\varepsilon} \lambda(x, \operatorname{dist}(x, y))}, \quad \operatorname{dist}(x, y) \geqslant C \operatorname{dist}\left(x, x^{\prime}\right), \\
\left|K(x, y)-K\left(x, y^{\prime}\right)\right| \leqslant C \frac{\operatorname{dist}\left(y, y^{\prime}\right)^{\varepsilon}}{\operatorname{dist}(x, y)^{\varepsilon} \lambda(y, \operatorname{dist}(x, y))}, \quad \operatorname{dist}(x, y) \geqslant C \operatorname{dist}\left(y, y^{\prime}\right) .
\end{gathered}
$$

Через $B(x, r)$, как обычно, обозначен шар в $\mathscr{X}: B(x, r)=\{y \in \mathscr{X}: \operatorname{dist}(x, y)<r\}$.

Пусть мера $\mu$ на $\mathscr{X}$ такова, что $\mu(B(x, r)) \leqslant \lambda(x, r)$, где $C$ не зависит от $x$ и $r$. В этом смысле $\lambda(x, r)$ есть локальная мажоранта для $\mu(B(x, r))$, удовлетворяющая условию удвоения. Мы будем говорить, что $T$ является оператором КЗ с ядром $K$, если $T$ ограничен из $L^{2}(\mu)$ в $L^{2}(\mu)$ и

$$
T f(x)=\int K(x, y) f(y) d \mu(y) \quad \forall x \notin \operatorname{supp} \mu .
$$

Для таких операторов верны все теоремы предыдущих разделов, т.е. верна как $L^{2}$, так и $L^{p}$-теория, включая слабый тип [44]. Это бывает полезно. Например, такое обобщение неоднородной теории КЗ было использовано в [128] для описания мер Карлесона в шаре для всей шкалы пространств Бесова-Харди. Ранее такое описание было возможно только для части шкалы - см. [104], [105], где использовалась некоторая разновидность кривизны Менгера. 


\section{4. Емкости Кальдерона-Зигмунда}

Теория операторов КЗ позволяет вводить ассоциированные с ними емкости не только в $\mathbb{R}^{2}$, но и в метрических пространствах. Целое семейство так называемых емкостей $\mathrm{K} 3$ в $\mathbb{R}^{d}, d \geqslant 2$, изучается в [126]. Их можно рассматривать как естественные аналоги аналитической емкости в $\mathbb{R}^{d}, d \geqslant 2$. Остановимся на некоторых из этих емкостей. Особое внимание мы уделим случаю операторов с ядрами

$$
\begin{aligned}
K_{i}(x, y) & =\frac{x_{i}-y_{i}}{|x-y|^{s+1}} \\
x=\left(x_{1}, \ldots, x_{d}\right) \in \mathbb{R}^{d}, \quad y & =\left(y_{1}, \ldots, y_{d}\right) \in \mathbb{R}^{d}, \quad x-y \neq 0,
\end{aligned}
$$

являющимися классическим и исключительно важным примером ядер К3 (с $\varepsilon=1$ и $A=A(d, s))$. Удобно рассматривать векторнозначное ядро Рисса

$$
K(x, y)=\frac{x-y}{|x-y|^{s+1}}, \quad x-y \neq 0,
$$

и соответствующий оператор Рисса

$$
R_{\nu}^{s} f(x)=\int \frac{x-y}{|x-y|^{s+1}} f(y) d \nu(y), \quad f \in L^{2}(\nu), \quad \nu \in M\left(\mathbb{R}^{d}\right),
$$

а также преобразование Рисса

$$
R^{s} \nu(x):=R_{\nu}^{s} \mathbf{1}(x)=\int \frac{x-y}{|x-y|^{s+1}} d \nu(y) .
$$

Нам понадобятся также их срезки

$$
R_{\nu, \varepsilon}^{s} f(x)=\int_{|x-y|>\varepsilon} \frac{x-y}{|x-y|^{s+1}} f(y) d \nu(y), \quad R_{\varepsilon}^{s} \nu(x):=R_{\nu, \varepsilon}^{s} \mathbf{1}(x) .
$$

В связи с задачами гармонической аппроксимации Парамонов [94] ввел понятие липшицевой гармонической емкости множества в $\mathbb{R}^{d}, d \geqslant 2$ :

$\kappa(E):=\sup \left\{|\langle\Delta f, 1\rangle|: f \in \operatorname{Lip}_{\text {loc }}^{1}\left(\mathbb{R}^{d}\right), \operatorname{supp}(\Delta f) \subset E,\|\nabla f\|_{\infty} \leqslant 1, \nabla f(\infty)=0\right\}$,

где $\operatorname{Lip}_{\text {loc }}^{1}\left(\mathbb{R}^{d}\right)$ - множество действительных локально липшицевых функций (с показателем 1$)$ в $\mathbb{R}^{d}$ и $\langle S, \varphi\rangle$ означает действие распределения (обобщенной функции) $S$ с компактным носителем на гладкую функцию $\varphi$. В [94], [71] отмечено, что $\kappa(E)=0$ тогда и только тогда, когда множество $E$ устранимо для липшицевых гармонических функций. Точнее говоря, когда для любой области $D \subset \mathbb{R}^{d}$ каждая локально липшицева функция $f: D \rightarrow \mathbb{R}$, гармоническая в $D \backslash E$, является также гармонической в $D$. Маттила и Парамонов [94], [71] установили важные геометрические свойства емкости $\kappa$ (отметим, что в [94], [71] липшицева гармоническая емкость $\kappa$ обозначается $\left.\kappa^{\prime}\right)$. В частности, они указали условия, достаточные для соизмеримости емкости $\kappa$ и меры Хаусдорфа $H^{d-1}$. С другой стороны, ими найден достаточно широкий класс компактов положительной $H^{d-1}$-меры и нулевой $\kappa$-емкости. 
Обозначим через $M_{+}$класс положительных радоновских мер в $\mathbb{R}^{d}$. В [71], [123] (см. также [126]) рассмотрена следующая модификация емкости $\kappa$ :

$$
\begin{gathered}
\kappa_{+}(E):=\sup \left\{|\langle\Delta f, 1\rangle|: f \in \operatorname{Lip}_{\text {loc }}^{1}\left(\mathbb{R}^{d}\right), \operatorname{supp}(\Delta f) \subset E,\|\nabla f\|_{\infty} \leqslant 1,\right. \\
\left.\nabla f(\infty)=0, \Delta f=\mu \in M_{+}\right\} .
\end{gathered}
$$

Очевидно, что $\kappa_{+}(E) \leqslant \kappa(E)$; нулевые множества емкости $\kappa_{+}(E)$ - это множества устранимых особенностей липшицевых субгармонических функций [123]. В терминах емкости $\kappa_{+}(E)$ в [123; теорема 1.1$]$ установлен критерий $C^{1}$-приближаемости на компакте $X \subset \mathbb{R}^{d}$ субгармонической в $\mathbb{R}^{d}$ функции $f$ функциями $f_{n}$, субгармоническими в $\mathbb{R}^{d}$ и гармоническими в окрестности (зависящей от $n$ ) компакта $X$. Отметим еще один результат, а именно полуаддитивность емкости $\kappa_{+}(E)$ [123; теорема 6.2]. Это свойство установлено в высших размерностях, когда метод кривизны меры не работает. Ниже, после наброска доказательства теоремы 4.1, мы получим обобщение этого утверждения: счетную полуаддитивность более широкого класса емкостей.

В [94] замечено, что $\kappa(E) \leqslant 2 \pi \gamma(E)$ при $d=2$. Так как функции $f$, участвующие в определении емкости $\kappa_{+}(E)$ при $d=2$, представимы в виде логарифмического потенциала по мере $c \mu$ и

$$
\nabla(\log |z|)=\left(\frac{x}{|z|^{2}}, \frac{y}{|z|^{2}}\right), \quad \frac{x}{|z|^{2}}+i \frac{y}{|z|^{2}}=\frac{1}{\bar{z}}, \quad z=x+i y,
$$

то $|\nabla f|=c|\mathscr{C} \mu|$. Поэтому $c \kappa_{+}(E)=\gamma_{+}(E)$. Таким образом, в силу теоремы Толсы [110] $\kappa_{+}(E) \approx \kappa(E), d=2$. Для $d>2$ это соотношение доказано в [126; теорема 2.1 и лемма 5.15].

В [94] введено также понятие $C^{1}$-гармонической емкости $\kappa^{c}$ (в определении емкости $\kappa$ вместо условия $f \in \operatorname{Lip}_{\text {loc }}^{1}\left(\mathbb{R}^{d}\right)$ берется $\left.f \in C^{1}\left(\mathbb{R}^{d}\right)\right)$. Важность этого понятия для изучения аппроксимации гармоническими функциями в $C^{1}$-норме продемонстрирована в [123]. Емкость $\kappa^{c}$ можно рассматривать как многомерное обобщение понятия непрерывной аналитической емкости в $\mathbb{C}$. В [100] показано, что емкость $\kappa^{c}$ соизмерима со своей положительной версией $\kappa_{+}^{c}$ (модификацией, аналогичной емкости $\left.\kappa_{+}\right)$. В [100] установлено также, что $\kappa_{+}^{c}$ счетно полуаддитивна. Отсюда следует, что этим свойством обладает и $\kappa^{c}$. Задачи $C^{m}$-аппроксимации и связанные с ними емкости подробно описаны в обзоре Мазалова, Парамонова и Федоровского [76].

Перейдем к следующему понятию емкости, содержащему $\gamma_{+}(E)$ и $\kappa_{+}(E)$ как частные случаи. Для заданного $s \in(0, d)$ емкость $\gamma_{s,+}(E)$ компакта $E \subset \mathbb{R}^{d}$ определяется равенством

$$
\gamma_{s,+}(E):=\sup \left\{\|\mu\|: \mu \in M_{+}, \operatorname{supp} \mu \subset E,\left\|R^{s} \mu\right\|_{L^{\infty}\left(\mathbb{R}^{d}\right)} \leqslant 1\right\} .
$$

Если $\|\mu\|<\infty, 0<s \leqslant d$, то предел $\lim _{\varepsilon \rightarrow 0} R_{\varepsilon}^{s} \mu(x)$ существует почти всюду относительно лебеговой меры в $\mathbb{R}^{d}$. Таким образом, данное определение корректно, если под $R^{s} \mu$ понимать этот предел. Из условия $\left\|R^{s} \mu\right\|_{L^{\infty}\left(\mathbb{R}^{d}\right)} \leqslant 1$ следует, что $\mu(B(x, r)) \leqslant C r^{s}$ для любого шара $B(x, r) \subset \mathbb{R}^{d}$ и $C=C(d, s)$ (см. [57; с. 217] или [35; раздел 5]; приведенные в этих работах аргументы работают при всех $d \geqslant 2,0<s<d)$. Поэтому условие $\mu \in M_{+}$в (4.2) можно 
заменить более сильным предположением $\mu \in \Sigma_{s}$; определяемая таким образом емкость соизмерима с $\gamma_{s,+}$.

Емкость $\gamma_{s,+}$ тесно связана с различными задачами анализа. При $d=2$, $s=1$ имеем

$$
\gamma_{1,+}(E)=\gamma_{+}(E) \approx \gamma(E)
$$

(равенство следует из очевидного соотношения $|\mathscr{C} \mu(z)|=\left|R^{1} \mu(z)\right|-$ см. (4.1)).

При $d \geqslant 2, s=d-1$ имеем

$$
\gamma_{d-1,+}(E) \approx \kappa_{+}(E) \approx \kappa(E)
$$

Первое соотношение вытекает из простого, но очень полезного равенства

$$
\nabla\left(\int \frac{d \mu(y)}{|x-y|^{s-1}}\right)=(1-s) R^{s} \mu(x), \quad s \neq 1
$$

(при $s=1$ следует взять градиент логарифмического потенциала, см. (4.1)). О втором соотношении в (4.3) уже сказано выше. В дальнейшем мы увидим, что $\gamma_{s,+}$ связана и с емкостями, возникающими в нелинейной теории потенциала.

Рассмотрим теперь емкость $\gamma_{s}$, называемую иногда $s$-мерной емкостью Кальдерона-Зигмунда:

$$
\gamma_{s}(E)=\sup |\langle T, 1\rangle|
$$

где верхняя грань берется по всем действительным распределениям $T$ с носителями в $E$, для которых

$$
\left\|\frac{x}{|x|^{s+1}} * T\right\|_{L^{\infty}\left(\mathbb{R}^{d}\right)} \leqslant 1, \quad x \in \mathbb{R}^{d} .
$$

Очевидно, что $\gamma_{s,+}(E) \leqslant \gamma_{s}(E), 0<s<d$.

При $d=2, s=1$ величина $\gamma_{s}$ соизмерима с аналитической емкостью $\gamma$, определение которой дано в начале раздела 2. Это требует объяснения. Прежде всего заметим, что $\gamma$ можно определить так же, как $\gamma_{1}$, если (4.5) заменить условием $\|(1 / z) * T\|_{L^{\infty}(\mathbb{C})} \leqslant 1$ и под $T$ понимать комплексные распределения. В самом деле, переход от $f$ к $T$ и обратно реализуется формулами $T=(1 / \pi) \bar{\partial} f$ и $f=(1 / z) * T$. Для действительных распределений условие $\|(1 / z) * T\|_{L^{\infty}(\mathbb{C})} \leqslant 1$, очевидно, эквивалентно (4.5) (см. (4.1)). Но для комплексных распределений это неверно: из выполнения последнего условия уже не следует (4.5). Поскольку в определении $\gamma$ распределения должны быть комплексными, мы получаем лишь оценку $\gamma_{1}(E) \leqslant \gamma(E)$. Обратное неравенство $\gamma(E) \leqslant C \gamma_{1}(E)$ весьма нетривиально; оно следует из неравенства $\gamma(E) \leqslant C \gamma_{+}(E)$ Толсы [110]. В самом деле, в определение $\gamma_{+}$входят лишь действительные меры, и поэтому $\gamma_{+}(E) \leqslant \gamma_{1}(E)$.

Пусть $d \geqslant 2, s=d-1$ (теперь $T$ и $f$ снова действительны). Полагая $T=\Delta f$ и $f=-\left(a_{d}|x|^{2-d}\right) * T$, получим эквивалентность определений $\gamma_{d-1}$ и $\kappa$. Таким образом, при $d \geqslant 2, s=d-1$ имеем

$$
\gamma_{s}(E) \approx \gamma_{s,+}(E)
$$


(см. (4.3)). В [57] это соотношение установлено для $0<s<1$. В [59], [116], [36] рассмотрены различные классы канторовых множеств и $s \in(0, d)$. Для произвольных компактов в $\mathbb{R}^{d}$ и $0<s<d$ соотношение (4.6) доказано Прат [98].

Если вместо $R^{s} \mu$ в (4.2) взять максимальное преобразование Рисса

$$
R^{\sharp} \mu(x)=\sup _{\varepsilon>0}\left|R_{\varepsilon}^{s} \mu(x)\right|, \quad x \in \mathbb{R}^{d},
$$

то получим емкость

$$
\gamma_{s, *}(E):=\sup \left\{\|\mu\|: \mu \in \Sigma_{s}, \operatorname{supp} \mu \subset E, R^{\sharp} \mu(x) \leqslant 1, x \in \mathbb{R}^{d}\right\} .
$$

Класс мер $\Sigma_{s}$ в этом определении можно заменить множеством $M_{+}$. Определенная таким образом величина соизмерима с $\gamma_{s, *}-$ см. замечание после (4.2). Но определение (4.7) удобнее для дальнейшего изложения. Справедливы неравенства

$$
\gamma_{s, *}(E) \leqslant \gamma_{s,+}(E) \leqslant C \gamma_{s, *}(E), \quad C=C(d, s) .
$$

Первое неравенство следует непосредственно из определений. Второе вытекает из оценки

$$
R^{\sharp} \mu(x) \leqslant\left\|R^{s} \mu\right\|_{L^{\infty}\left(\mathbb{R}^{d}\right)}+C, \quad x \in \mathbb{R}^{d}, \quad \mu \in \Sigma_{s}, \quad C=C(d, s)
$$

(см. [124; лемма 2], [126; с. 47] или [1; лемма 5.1]).

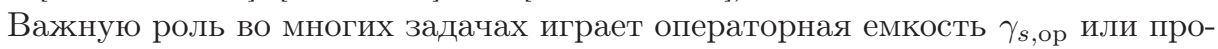
сто $\gamma_{\text {ор }}$ :

$$
\gamma_{\text {op }}(E):=\sup \left\{\|\mu\|: \mu \in \Sigma_{s}, \operatorname{supp} \mu \subset E,\left|R_{\mu}^{s}\right| \leqslant 1\right\}, \quad 0<s<d,
$$

где

$$
\left|R_{\mu}^{s}\right|=\left\|R_{\mu}^{s}\right\|_{L^{2}(\mu) \rightarrow L^{2}(\mu)}:=\sup _{\varepsilon>0}\left\|R_{\mu, \varepsilon}^{s}\right\|_{L^{2}(\mu) \rightarrow L^{2}(\mu)} .
$$

Согласно теореме $2.3, \gamma_{\text {op }}(E) \approx \gamma_{+}(E)$ при $d=2, s=1$. В [126; гл. 5] установлен более общий факт.

Tеорема 4.1. Для любого компакта $E \subset \mathbb{R}^{d}$

$$
\gamma_{s, *}(E) \approx \gamma_{s, \mathrm{op}}(E), \quad 0<s<d,
$$

с константами сравнения, зависящими только от $d u s$.

НАБРОСОК ДОКАЗАТЕЛЬСТВА. 1. Начнем с неравенства

$$
\gamma_{s, \mathrm{op}}(E) \leqslant C \gamma_{s, *}(E), \quad C=C(d, s)
$$

Для любой меры $\mu$, участвующей в (4.9), существует функция $h$ такая, что

$$
0 \leqslant h \leqslant 1, \quad \int_{E} h d \mu \geqslant \alpha \mu(E), \quad\left\|R_{\mu}^{s} h\right\|_{L^{\infty}(\mu)} \leqslant D,
$$

где $\alpha, D$ - положительные постоянные, зависящие только от $s$ и $d$. Доказательство этого важного факта имеется в [88; теорема 2.1] и в [126; теорема 5.13]. 
Оно использует аналогичное утверждение об операторе слабого типа [71; лемма 4.2], [126; лемма 5.11], [93; с. 82-83], элегантное доказательство которого основано на теореме Хана-Банаха о разделяющем функционале. Это утверждение (в менее общем виде) принадлежит Нгуену Ую [118], [119], исследовавшему устранимые особенности липшицевых ограниченных аналитических и гармонических функций. Упрощенное доказательство имеется также в [120; теорема 1.3], где теорема Нгуена Уя применяется в задаче о $C^{m}$-аппроксимации решениями эллиптических уравнений.

Для $f \in L^{1}(\mu)$ определим (модифицированную) максимальную функцию равенством

$$
\widetilde{M} f(x):=\sup _{r>0} \frac{1}{\mu(B(x, 3 r))} \int_{B(x, r)}|f| d \mu .
$$

Из оценки

$$
R_{\mu}^{\sharp} h(x) \leqslant C^{\prime} \widetilde{M}\left(R_{\mu}^{s} h(x)\right)+C^{\prime \prime}\left[\widetilde{M}\left(|h|^{2}\right)(x)\right]^{1 / 2}, \quad x \in \operatorname{supp} \mu,
$$

типа неравенства Котляра, доказанной в [87; теорема 7.1]), получаем

$$
R_{\mu}^{\sharp} h(x) \leqslant C, \quad x \in \operatorname{supp} \mu .
$$

Здесь постоянные $C^{\prime}, C^{\prime \prime}, C$ зависят только от $d$ и $s$. Это неравенство с помощью несложных аргументов приводит к оценке

$$
R_{\mu}^{\sharp} h(x) \leqslant C, \quad x \in \mathbb{R}^{d},
$$

с другой постоянной $C$ - см., например, $[1 ; \S 5]$. Определим меру $\sigma$ равенством

$$
d \sigma(x)=C_{1}^{-1} h(x) d \mu(x), \quad \text { где } \quad C_{1}=\max (C, 1)
$$

(здесь $C$ - постоянная из (4.12)). Тогда $\sigma \in \Sigma_{s}$ и согласно $(4.12) R^{\sharp} \sigma(x) \leqslant 1$, $x \in \mathbb{R}^{d}$. Таким образом, $\sigma$ участвует в (4.7). Следовательно,

$$
\gamma_{s, *}(E) \geqslant\|\sigma\|=C_{1}^{-1} \int h d \mu \geqslant \alpha_{1} \mu(E), \quad \alpha_{1}=\alpha_{1}(d, s)>0
$$

и мы получаем (4.11) с $C=\alpha_{1}^{-1}$.

2. Докажем теперь обратное неравенство

$$
\gamma_{s, *}(E) \leqslant C \gamma_{s, \mathrm{op}}(E) .
$$

Возьмем меру $\mu$, участвующую в (4.7), и зафиксируем $\varepsilon>0$. Очевидно, что $\left|R_{\varepsilon}^{s} \mu(x)\right|=\left|R_{\mu, \varepsilon}^{s} \mathbf{1}(x)\right| \leqslant 1$, однако мы не можем применить $\mathrm{k} R_{\varepsilon}^{s} \mu$ инструментарий теории K3, поскольку срезанное ядро не является ядром КЗ. Стандартный прием состоит в переходе к оператору со сглаженным ядром, являющемуся оператором КЗ. С этой целью возьмем $C^{\infty}$-функцию $\phi(t), t \geqslant 0$, такую, что $\phi(t)=0$ при $0 \leqslant t \leqslant 1, \phi(t)=1$ при $t \geqslant 2$ и $0 \leqslant \phi^{\prime}(t) \leqslant 2$ при $t>0$. Положим $\phi_{\varepsilon}(t)=\phi(t / \varepsilon)$. Непосредственная проверка показывает (см., например, $[1 ; \S 4])$, что если $K(x, y)$ - ядро КЗ с константами $A, s, \delta$, то $K_{\varepsilon}(x, y):=\phi_{\varepsilon}(|x-y|) K(x, y)-$ ядро КЗ с константами $9 A, s, \delta$. Поэтому

$$
\widetilde{R}_{\mu, \varepsilon}^{s} f(x)=\int_{\mathbb{R}^{d}} \phi_{\varepsilon}(|x-y|) \frac{y-x}{|y-x|^{s+1}} d \mu(y)
$$


является оператором КЗ. Нетрудно убедиться, что при $\mu \in \Sigma_{s}$ и $f \in L^{1}(\mu)$ справедлива оценка

$$
\left|R_{\mu, \varepsilon}^{s} f(x)-\widetilde{R}_{\mu, \varepsilon}^{s} f(x)\right| \leqslant C \widetilde{M} f(x), \quad x \in \mathbb{R}^{d},
$$

где $C$ зависит от параметров ядра $K$ (в нашем случае от $s$ ). В частности, при $f(x)=1$ получаем

$$
\left|\widetilde{R}_{\mu, \varepsilon}^{s} \mathbf{1}(x)\right| \leqslant C, \quad x \in \mathbb{R}^{d} .
$$

Следовательно, $\widetilde{R}_{\mu, \varepsilon}^{s} \mathbf{1}(x) \in L^{\infty}(\mu)$, и к оператору $\widetilde{R}_{\mu, \varepsilon}^{s}$ применима $T(1)$-теорема из [90] для неоднородных пространств, согласно которой

$$
\left\|\widetilde{R}_{\mu, \varepsilon}^{s}\right\|_{L^{2}(\mu) \rightarrow L^{2}(\mu)} \leqslant C, \quad C=C(d, s) .
$$

Максимальный оператор $\widetilde{M}$ ограничен как оператор из $L^{2}(\mu)$ в $L^{2}(\mu)-$ см. [87; лемма 3.1]. Поэтому в силу (4.14)

$$
\left\|R_{\mu, \varepsilon}^{s}\right\|_{L^{2}(\mu) \rightarrow L^{2}(\mu)} \leqslant C, \quad C=C(d, s)
$$

(заметим, что $C$ не зависит от $\varepsilon$ ). Значит, мера $C^{-1} \mu$ участвует в $(4.9)$, и мы приходим к (4.13).

Мы изложили идею доказательства теоремы 4.1 по двум причинам. Во-первых, чтобы продемонстрировать характерные методы, работающие при доказательстве целого ряда утверждений теории КЗ. Во-вторых, из-за важности этой теоремы: операторная емкость является, пожалуй, наиболее удобной для работы среди всего семейства емкостей К3, рассмотренных выше. Для иллюстрации этого тезиса заметим, что если оператор $R_{\mu}^{s}$ ограничен (т. е. $\left|R_{\mu}^{s}\right| \leqslant C$ ), то для любого компакта $E \subset \operatorname{supp} \mu$ оператор $R_{\mu \mid E}^{s}$ также ограничен с той же константой. Отсюда и из (4.10), (4.8), (4.6) сразу следует счетная полуаддитивность всех изучаемых емкостей. Отметим, что ограниченность преобразования Рисса (т. е. $\left\|R^{s} \mu\right\|_{L^{\infty}\left(\mathbb{R}^{d}\right)} \leqslant C$ ) аналогичным свойством не обладает. Значение теоремы 4.1 состоит также в том, что она сводит оценку емкости к соответствующей задаче для операторной нормы, которая во многих случаях успешно решается. Ряд таких применений излагается ниже. Соизмеримость емкостей $\gamma_{\text {ор }}$ и $\kappa_{+}\left(\right.$а значит, и $\gamma_{+}$при $d=2$ ) рассматривалась и в [90]. В этой работе, а также в $[123 ; \S 1]$, имеется физическая интерпретация задач, связанных с емкостью $\kappa_{+}$.

Обобщения. Уже говорилось, что многие понятия теории сингулярных интегралов вводятся для ядер КЗ в метрических пространствах с геометрическим условием удвоения: каждый открытый шар $B(x, r)$ можно покрыть не более чем $N$ шарами радиуса $r / 2$, причем конечное число $N$ не зависит от $x, r$. В [1] рассматриваются емкости в этих пространствах, порожденные операторами K3 с более общими ядрами, чем ядра Рисса, и доказываются теоремы сравнения (в частности, обобщается теорема 4.1).

В [58] вводятся и изучаются емкости $\Gamma_{\widehat{k}}$, порожденные компонентами $x_{i} /|x|^{2}$ ядра $x /|x|^{2}, x=\left(x_{1}, \ldots, x_{d}\right) \in \mathbb{R}^{d}$. В определении $\Gamma_{\widehat{k}}$ требуется, чтобы $d-1$ компонент векторного потенциала $\left(x /|x|^{2}\right) * T$ были ограничены единицей в $L^{\infty}\left(\mathbb{R}^{d}\right)$. 
В частности, показано, что при $d=2$ эти емкости соизмеримы с аналитической емкостью $\gamma$. Более того, при $d>2$ и дополнительном условии, что распределения $T$ имеют линейный рост, справедливо соотношение $\Gamma_{\widehat{k}} \approx \gamma_{1}$. Но соответствующее утверждение для ядра $x /|x|^{s+1}$ не имеет места при $d>2$, $0<s<1$ ! В [58] имеются и другие контрпримеры. Интересное развитие этих результатов получено в [16]. А именно, вводятся емкости, ассоциированные с ядрами $x_{i}^{2 n-1} /|x|^{2 n}, i=1,2, x=\left(x_{1}, x_{2}\right) \in \mathbb{R}^{2}, n \geqslant 1$, и устанавливается их соизмеримость с $\gamma$. В случае $d>2, n=1$ доказано соотношение $\Gamma_{\widehat{k},+} \approx \gamma_{1}$, где $\Gamma_{\widehat{k},+}-$ версия емкости $\Gamma_{\widehat{k}}$, в определении которой условия роста распределений $T$ отсутствуют, но распределения заменены положительными мерами. В [15] используется аналог кривизны меры для ядер $K_{n}(z)=x^{2 n-1} /|z|^{2 n}$, $z=x+i y \in \mathbb{C} \backslash\{0\}$, и доказывается аналог теоремы 2.4. Это первый пример оператора с ядром, существенно отличным от ядра Коши, ограниченность которого влечет 1-спрямляемость множества $E$.

\section{5. Оценки преобразования Рисса и емкостей. Емкость и мера Хаусдорфа}

Мы начнем с оценки $L^{2}(\mu)$-нормы оператора Рисса, важность которой ясна из предыдущего раздела. Естественно попытаться использовать те же приемы, которые работали в случае преобразования Коши, а именно T(1)-теорему и равенство (2.3), основанное на (2.4). Мы уже видели в разделе 3 , что неоднородные $T(1)$ - и $T(b)$-теоремы доказаны в весьма общих предположениях. Покажем, как возникает многомерный аналог левой части равенства (2.4). Для простоты изложения ограничимся случаем, когда интеграл $R^{s} \mu(x)$ сходится абсолютно при $x \in \mathbb{R}^{d}$, так что рассматривать срезанные ядра нет необходимости. Имеем

$$
\begin{aligned}
\left\|R^{s} \mu\right\|_{L^{2}(\mu)}^{2} & =\int\left|\int \frac{y-x}{|y-x|^{s+1}} d \mu(y)\right|^{2} d \mu(x) \\
& =\iiint \frac{y-x}{|y-x|^{s+1}} \frac{z-x}{|z-x|^{s+1}} d \mu(y) d \mu(z) d \mu(x)
\end{aligned}
$$

(здесь берется обычное скалярное произведение). Предполагая условия теоремы Фубини выполненными, получим

$$
\left\|R^{s} \mu\right\|_{L^{2}(\mu)}^{2}=\frac{1}{6} \iiint \sum_{s} \frac{x_{\sigma(2)}-x_{\sigma(1)}}{\left|x_{\sigma(2)}-x_{\sigma(1)}\right|^{s+1}} \frac{x_{\sigma(3)}-x_{\sigma(1)}}{\left|x_{\sigma(3)}-x_{\sigma(1)}\right|^{s+1}} d \mu(x) d \mu(y) d \mu(z),
$$

где, как и ранее, через $\sigma$ обозначены перестановки индексов $1,2,3$ (на самом деле только три перестановки дают различные слагаемые в (5.1), а иногда удобнее обойтись и двумя - см. [34]). Если рассуждать аккуратно, то следует рассмотреть срезанное преобразование Рисса $R_{\varepsilon}^{s} \mu(x)$ и применить стандартный прием симметризации области интегрирования для получения аналога (2.3) см., например, [81], [112], [113]. Но на следующем этапе - при оценке суммы в (5.1) - нас ждет тяжелый удар. Мало того, что при $s \neq 1$ отсутствует замечательная геометрическая интерпретация, подобная (2.4). Оказывается, что при $s>1$ не для всех троек $x_{1}, x_{2}, x_{3}$ сумма в (5.1) будет положительной! 
(Как показал Фараг [38], при $s>1$ такая ситуация имеет место для широкого класса функций троек $x_{1}, x_{2}, x_{3}$ типа суммы в (5.1).) Насколько нам известно, попытки оценить вклад "положительных" и "отрицательных" троек оказались безрезультатными. Именно в этом заключается причина того, что наиболее полные результаты получены в случае $0<s \leqslant 1$, когда сумма в (5.1) неотрицательна (а при $0<s<1$ положительна) для любых троек $x_{1}, x_{2}, x_{3}$. Тем не менее оценку сверху этой суммы удается вывести для всех $s \in(0, d)$. Следствием этой оценки и $T(1)$-теоремы 3.2 Назарова-Треиля-Вольберга [86] является следующее утверждение.

Теорема 5.1 [34]. Для любой конечной неотрицательной борелевской меры $\mu в \mathbb{R}^{d}$ и $0<s<d$ справедлива оценка

$$
\left|R_{\mu}^{s}\right|^{2} \leqslant C \sup _{x \in \operatorname{supp} \mu} W^{\mu}(x), \quad \text { əде } \quad W^{\mu}(x):=\int_{0}^{\infty}\left[\frac{\mu(B(x, r))}{r^{s}}\right]^{2} \frac{d r}{r}
$$

и $C$ зависит только от $d u s$.

Функция $W^{\mu}(x)$ называется потенциалом Вольфа.

В данном разделе мы рассмотрим три применения теоремы 5.1.

\section{1. Оценка емкостей Кальдерона-Зигмунда. Связь с емкостями} из нелинейной теории потенциала. Из теорем 4.1 и 5.1 нетрудно вывести следующий результат (см. [34; теорема 2.7]).

Tеорема 5.2. Для любого компакта $E \subset \mathbb{R}^{d} u 0<s<d$

$$
\gamma_{s,+}(E) \geqslant c \sup \|\mu\|^{3 / 2}\left[\int_{\mathbb{R}^{d}} W^{\mu}(x) d \mu(x)\right]^{-1 / 2},
$$

где верхняя грань берется по всем положительным радоновским мерам с носителем в Е и с зависит только от $d, s$.

При $0<s<1$ имеет место и обратное неравенство. По существу, это установлено в [57], обобщение см. в [1; теорема 2.1]. Из дальнейшего будет ясно, что при натуральных $s$ обратное неравенство, вообе говоря, неверно.

Потенциал Вольфа является мостиком, связывающим емкости КЗ с емкостями из нелинейной теории потенциала [2], [77]. Одно из нескольких эквивалентных определений состоит в следующем [2; теорема 2.5.1]:

$$
\dot{C}_{\alpha, p}(E)=\sup _{\mu \in M_{+}(E)}\left(\frac{\mu(E)}{\left\|I_{\alpha} * \mu\right\|_{p^{\prime}}}\right)^{p}, \quad I_{\alpha}(x)=\frac{A_{d, \alpha}}{|x|^{d-\alpha}}, \quad \frac{1}{p^{\prime}}+\frac{1}{p}=1,
$$

где $M_{+}(E)$ означает класс мер из $M_{+}$с носителем в $E, 1<p<\infty, 0<\alpha p<d$, через $\|\cdot\|_{p^{\prime}}$ обозначена $L^{p^{\prime}}$-норма относительно лебеговой меры в $\mathbb{R}^{d}$ и постоянная $A_{d, \alpha}$ зависит только от $d$ и $\alpha$. Ясно, что $\left(I_{\alpha} * \mu\right)(x) \approx\|\mu\| \cdot|x|^{\alpha-d}$ для конечной меры $\mu$ с компактным носителем и достаточно больших значений $|x|$. Если $\alpha p=d$, то $p^{\prime}=d /(d-\alpha)$, и, следовательно, $I_{\alpha} * \mu \notin L^{p^{\prime}}$. В частности, поэтому в [2] преимущественно рассматривается емкость Бесселя $C_{\alpha, p}$, определяемая так же, как и $\dot{C}_{\alpha, p}$, но с ядром Бесселя $G_{\alpha}(x)$ вместо ядра Рисса $I_{\alpha}(x)$. 
Мы не приводим определение и свойства ядра и потенциала Бесселя, отсылая читателя к монографии [2; с. 9-13]. Для нас важно, что

$$
G_{\alpha}(x) \approx I_{\alpha}(x) \quad \text { при }|x| \rightarrow 0, \quad 0<\alpha<d,
$$

и

$$
G_{\alpha}(x)=O\left(e^{-c|x|}\right) \quad \text { при }|x| \rightarrow \infty, \quad \alpha>0, \quad 0<c<1 .
$$

Поэтому $\dot{C}_{\alpha, p}(E) \approx C_{\alpha, p}(E)$ для компактов $E$ с $\operatorname{diam} E \leqslant 1$, где $1<p<\infty$, $0<\alpha p<d$. Но, в отличие от $\dot{C}_{\alpha, p}$, емкость $C_{\alpha, p}$ определена и при $\alpha p=d$.

В [57] доказано, что

$$
\gamma_{s,+}(E) \approx \dot{C}_{2(d-s) / 3,3 / 2}(E), \quad 0<s<1 .
$$

Односторонняя оценка имеет место при всех $s \in(0, d)-$ см. [34; следствие 10.1]:

$$
\gamma_{s,+}(E) \geqslant c \cdot \dot{C}_{2(d-s) / 3,3 / 2}(E), \quad 0<s<d .
$$

Соотношения (5.5), (5.6) нетрудно вывести из (5.3) (и обратного к нему неравенства при $0<s<1)$, применяя следующий результат Вольфа [2; с. 110 , теорема 4.5.4]: для любой меры $\mu \in M_{+}$и $1<p<\infty, 0<\alpha p<d$,

$$
\int_{\mathbb{R}^{d}} \dot{W}_{\alpha, p}^{\mu}(x) d \mu(x) \approx\left\|I_{\alpha} * \mu\right\|_{p^{\prime}}^{p^{\prime}}, \quad \dot{W}_{\alpha, p}^{\mu}(x):=\int_{0}^{\infty}\left[\frac{\mu(B(x, r))}{r^{d-\alpha p}}\right]^{p^{\prime}-1} \frac{d r}{r} .
$$

Полагая $\alpha=2(d-s) / 3, p=3 / 2$, получим $p^{\prime}=3, d-\alpha p=s$ и $\dot{W}_{\alpha, p}^{\mu}(x)=$ $W^{\mu}(x)$. Отсюда и из (5.4) следуют $(5.5)$ и (5.6) (см. [34; с. 757]). Аналогичное соотношение справедливо и для $G_{\alpha}$ вместо $I_{\alpha}$; в этом случае $\alpha p \leqslant d$ и последний интеграл в (5.7) берется от 0 до 1. Первый интеграл в (5.7) называется энергией Вольфа.

Обратное неравенство $\gamma_{s,+}(E) \leqslant C \cdot \dot{C}_{2(d-s) / 3,3 / 2}(E)$, вообще говоря, неверно при целых $s \in(0, d)$. Например, замкнутый $s$-мерный шар $E$ в $\mathbb{R}^{d}$ имеет положительную $\gamma_{s,+}$-емкость и нулевую $\dot{C}_{2(d-s) / 3,3 / 2}$-емкость. Действительно, оператор Рисса с обычной лебеговой мерой на $E$ ограничен (см. [102; гл. 6]), и согласно $(4.8)$ и $(4.10) \gamma_{s,+}(E)>0$. С другой стороны, нетрудно заметить (см., например, [57]), что любая мера $\mu$ с конечной энергией Вольфа удовлетворяет равенству $\mu(B(x, r))=o\left(r^{s}\right)$ для $\mu$-почти всех $x$. На $s$-мерном шаре таковой является только нулевая мера; таким образом, $\dot{C}_{2(d-s) / 3,3 / 2}(E)=0$.

Вопрос о справедливости неравенства $\gamma_{s,+}(E) \leqslant C \cdot \dot{C}_{2(d-s) / 3,3 / 2}(E)$ для нецелых $s \in(0, d)$ остается открытым. Некоторые недавние продвижения в этой задаче обсуждаются ниже в разделе 7.

Емкость $C_{\alpha, p}$ можно определить следующим образом [2], [77]:

$$
C_{\alpha, p}(E)=\inf \left\{\|\varphi\|_{\alpha, p}^{p}: \varphi \in S, \varphi \geqslant 1 \text { на } E\right\},
$$

где $S$ - класс Шварца и $\|\cdot\|_{\alpha, p}$ - норма в пространстве Соболева $W^{\alpha, p}$. В частности, в силу (5.6) получаем, что аналитическая емкость $(d=2, s=1)$ ограничена снизу емкостью, порожденной действительными функциями на плоскости, 
у которых производные порядка $2 / 3$ лежат в $L^{3 / 2}$ - весьма неожиданный факт! Ряд интересных применений емкости $C_{\alpha, p}$ описан в [78].

В [1] рассматривается следующий вопрос: можно ли ввести естественный аналог емкости $\gamma_{s,+}$, который был бы соизмеримым с $C_{2(d-s) / 3,3 / 2}$ при целых $s$ ? С этой целью понятие емкости $\gamma_{s,+}$ обобщается следующим образом. Пусть $\varphi(t), t \geqslant 0,-$ непрерывная возрастающая функция с $\varphi(0)=0$. Емкость $\gamma_{\varphi,+}$ компакта $E \subset \mathbb{R}^{d}$ определяется равенством

$$
\gamma_{\varphi,+}(E):=\sup \left\{\|\mu\|: \mu \in M_{+}(E), \mu(B(x, r)) \leqslant \varphi(r),\left\|R_{\mu}^{\varphi}\right\|_{L^{\infty}\left(\mathbb{R}^{d}\right)} \leqslant 1\right\},
$$

где

$$
R_{\mu}^{\varphi}(x)=\int \frac{y-x}{|y-x|} \frac{1}{\varphi(|y-x|)} d \mu(y) .
$$

Очевидно, что $\gamma_{s,+}(E) \approx \gamma_{\varphi,+}(E)$ при $\varphi(t)=t^{s}$. В [1] обобщается ряд результатов, описанных выше. В качестве следствия получен аналог соотношения (5.5) для $s=0$ : если

$$
\varphi(t)=\left(\log \frac{1}{t}\right)^{-1 / 2}, \quad 0<t \leqslant e^{-3 / 2},
$$

и $\varphi$ “правильно" продолжена на весь интервал $(0, \infty)$, то

$$
\gamma_{\varphi,+}(E) \approx C_{2 d / 3,3 / 2}(E)
$$

для любого компакта $E$ c $\operatorname{diam} E \leqslant 1$. Выдвигается гипотеза, что при натуральных $s$ не существует функций $\varphi$, для которых

$$
\gamma_{\varphi,+} \approx C_{2(d-s) / 3,3 / 2}
$$

5.2. Емкости Кальдерона-Зигмунда и мера Хаусдорфа. Назовем функцию $h:[0,+\infty) \rightarrow[0,+\infty)$ измеряющей, если она непрерывна, строго возрастает, $h(0)=0$ и $\lim _{r \rightarrow+\infty} h(r)=+\infty$. Обхват по Хаусдорфу $M_{h}(G)$ множества $G \subset \mathbb{R}^{d}$ определяется равенством

$$
M_{h}(G)=\inf \sum_{j} h\left(r_{j}\right),
$$

где нижняя грань берется по всем (не более чем счетным) покрытиям множества $G$ шарами с радиусами $r_{j}$. Для дальнейшего удобно предполагать, что функция $h(r) / r^{d}$ не возрастает. Это условие, кажущееся на первый взгляд дополнительным условием регулярности, на самом деле не является ограничением. В [2; с. 133, предложение 5.18] показано, что для любой измеряющей функции $h$ либо $M_{h}(G)=0$ для всех $G \subset \mathbb{R}^{d}$, либо существует другая ("исправленная") измеряющая функция $h^{*}$ такая, что $h^{*}(r) / r^{d}$ не возрастает и обхваты по Хаусдорфу $M_{h}$ и $M_{h^{*}}$ совпадают с точностью до постоянного множителя, зависящего только от размерности $d$. Отметим, что обхват $M_{h}$ и мера Хаусдорфа $H^{h}$ обращаются в нуль одновременно.

Из неравенства (5.3) и классической теоремы Фростмана (см., например, [13; гл. II, теорема 1]) легко выводится следующее утверждение [34; с. 754, теорема 10.1]. 
TEOPEMA 5.3. Пусmь

$$
\int_{0}\left(\frac{h(t)}{t^{s}}\right)^{2} \frac{d t}{t}<\infty
$$

Тогда для любого компакта $E \subset \mathbb{R}^{d}$

$$
\gamma_{s,+}(E) \geqslant c M_{h}(E)\left[\int_{0}^{t_{2}}\left(\frac{h(t)}{t^{s}}\right)^{2} \frac{d t}{t}\right]^{-1 / 2}, \quad 0<s<d
$$

где с зависит только от $d u s$, a $t_{2}$ определяется равенством $h\left(t_{2}\right)=M_{h}(E)$.

Аналог теоремы 5.3 имеет место и для емкости $\dot{C}_{\alpha, p}$ :

$$
\dot{C}_{\alpha, p}(E) \geqslant c M_{h}(E)\left[\int_{0}^{t_{2}}\left(\frac{h(t)}{t^{d-\alpha p}}\right)^{p^{\prime}-1} \frac{d t}{t}\right]^{-p / p^{\prime}}, \quad 0<\alpha p<d, \quad p>1 .
$$

Его можно вывести, используя рассуждения из [77], [2; гл. 5] и [34; с. 755]. Из этой оценки и (5.6) опять получается (5.9).

Применяя теорему 5.3, получим следующую оценку [34; с. 755]:

$$
c(\beta-s)^{1 / 2}\left[M_{t^{\beta}}(E)\right]^{s / \beta} \leqslant \gamma_{s,+}(E) \leqslant C M_{t^{s}}(E), \quad 0<s<d, \quad \beta>s,
$$

где постоянные $c, C$ зависят только от $d$ и $s$. Первое неравенство сразу следует из $(5.9)$ при $h(t)=t^{\beta}$. Оно уточняет результат Прат из статьи [96; с. 946], в которой получено и второе неравенство в (5.10).

СлеДСтвиЕ 5.4. Для $s \in(0, d)$ и любого компакта $E \subset \mathbb{R}^{d}$ справедливы следующие утверждения:

1) если $\gamma_{s,+}(E)>0$, mо $H^{h}(E)>0$ для $h(t)=t^{s}$;

2) если $H^{h}(E)>0$, то $\gamma_{s,+}(E)>0$ для любой измеряющей функции $h$, удовлетворяющей (5.8).

Изложенные выше результаты являются в некотором смысле точными. Так, в первой части следствия 5.4 функцию $t^{s}$ нельзя заменить функцией $h(t)=o\left(t^{s}\right)$ (см. [34; с. 755]). Условие (5.8) во второй части следствия 5.4 необходимо (здесь существенно, что функция $h(r) / r^{d}$ не возрастает) - см. следующий раздел. Поэтому зазор между частями 1 и 2 уменьшить нельзя. Следствие 5.4 можно рассматривать как аналог классической теоремы Фростмана [13; гл. IV, теорема 1] о связи между емкостью, порожденной обычными потенциалами Рисса с ядром $1 / r^{s}$, и мерой Хаусдорфа. Интересно отметить, что критическую роль здесь играет сходимость интеграла $\int_{0} \frac{h(t)}{t^{s}} \frac{d t}{t}-\mathrm{cp.} \mathrm{c} \mathrm{(5.8).} \mathrm{Очевидно,} \mathrm{что} \mathrm{из}$ условия $\int_{0} \frac{h(t)}{t^{s}} \frac{d t}{t}<\infty$ следует (5.8), но не наоборот. В этом проявляется эффект “сокращения", возникающий при замене положительного ядра $1 /|x|^{s}$ ядром $x /|x|^{s+1}$.

Результаты настоящего п. 5.2 обобщают утверждения из [31]-[33]. 
5.3. Оценки картановского типа. Интересным приложением излагаемой теории является решение старой задачи, поставленной Макинтайром и Фуксом в 1940 г. и, на первый взгляд, никак с этой теорией не связанной: для заданных $P>0$ и точек $z_{1}, \ldots, z_{N} \in \mathbb{C}$ найти точную оценку обхвата $M_{h}(\mathscr{Z})$, $h(t)=t$, множества

$$
\mathscr{Z}=\left\{z \in \mathbb{C}:\left|\sum_{k=1}^{N} \frac{1}{z-z_{k}}\right|>P\right\}
$$

Здесь сумма является логарифмической производной многочлена $\prod_{k=1}^{N}\left(z-z_{k}\right)$, и задача имеет приложение в теории мероморфных функций. История этой задачи изложена в [4], [5], где показано, что

$$
M_{t}(\mathscr{Z})<\frac{C}{P} N(\log N)^{1 / 2},
$$

и эта оценка неулучшаема (с точностью до константы $C$ ). Аналогичная задача об оценке суммы модулей простейших дробей решается гораздо проще: можно использовать известный метод Картана, примененный им для оценки снизу модуля многочлена. В этом случае правая часть (5.11) будет иметь вид $(C / P) N \log N$ и оценка также точна. Таким образом, показатель $1 / 2$ отражает эффект взаимного погашения слагаемых в сумме $\sum_{k=1}^{N} \frac{1}{z-z_{k}}$. Доказательство в [4], [5] основано на оценке емкости $\gamma_{+}$в терминах кривизны меры (см. раздел 2) и на оценке слабого типа для максимального преобразования Коши $\mathscr{C}^{\sharp} \mu(z)=\sup _{\varepsilon>0}\left|\mathscr{C}_{\varepsilon} \mu(z)\right|$.

В более общей постановке на плоскости задача Макинтайра-Фукса рассматривалась в [32], [33]. В [34] эти результаты перенесены на многомерный случай. Пусть $\nu$ - мера, состоящая из $N \geqslant 2$ комплексных точечных зарядов. Для заданных $P>0$ и $s>0$ положим

$$
\mathscr{Z}^{*}(\nu, P)=\left\{x \in \mathbb{R}^{d}: R_{\nu}^{\sharp}(x)>P\right\}
$$

и $\mathbf{M}:=M_{h}\left(\mathscr{Z}^{*}(\nu, P)\right)$ В [34] получена оценка для $\mathbf{M}$ в неявном виде:

$$
\mathbf{M} \leqslant C(s, d) \frac{\|\nu\|}{P}\left[\int_{h^{-1}(0.1 \mathrm{M} / N)}^{h^{-1}(\mathbf{M})}\left(\frac{h(t)}{t^{s}}\right)^{2} \frac{d t}{t}\right]^{1 / 2}
$$

где $h^{-1}$ - обратная к измеряющей функции $h$. Эту оценку можно записать в более простой форме, используя соответствующую вспомогательную функцию; подробнее см. [34]. Для "хороших функций” из (5.12) выводятся явные оценки. В частности, если $h(t)=t$ и $\nu$ состоит из единичных зарядов, получаем (5.11). При условии (5.8) справедлив "предельный случай" неравенства (5.12) при $N \rightarrow \infty$. А именно, в этом случае для любой меры (заряда) $\nu$ справедлив аналог оценки (5.12), в котором нижний предел интеграла равен нулю. Для точечных зарядов в [34] рассмотрен и случай $s \geqslant d$. Оказывается, 
что при этом оценка величины $\mathbf{M}$ та же (с точностью до постоянного множителя), что и для потенциала с ядром $1 / r^{s}$. Таким образом, при $s \geqslant d$ эффект взаимного сокращения слагаемых отсутствует.

Доказательство оценки (5.12) опирается на неравенство (5.2) и на следующий результат из [87]. Пусть $\mu \in \Sigma_{s}$ и $\left|R_{\mu}^{s}\right|<\infty$. Тогда для любой радоновской комплексной меры $\nu$ выполнено неравенство

$$
\mu\left\{x \in \mathbb{R}^{d}: R_{\nu}^{\sharp}(x)>t\right\}<\frac{C\|\nu\|}{t}
$$

с константой $C$, зависящей только от $s$ и $\left|R_{\mu}^{s}\right|$. Основным шагом в доказательстве (5.12) является построение вспомогательной меры $\mu$, позволяющей вывести эту оценку из (5.13) и (5.2).

\section{6. Канторовы множества}

Канторовы множества имеют большое значение для демонстрации точности различных оценок, а также для проверки тех или иных гипотез. Наиболее часто используются "угловые" канторовы множества в $\mathbb{R}^{d}$, при построении которых $j$-я генерация состоит из $2^{d j}$ кубов $E_{j, k}$ с ребром $\sigma_{j}$, каждый из кубов $E_{j, k}$ содержит $2^{d}$ кубов $(j+1)$-й генерации, расположенных в углах этого куба, и т. д. Здесь $\sigma_{0}, \sigma_{1}, \ldots$ - заданная последовательность положительных чисел такая, что $2 \sigma_{j+1} \leqslant \sigma_{j}, j=0,1, \ldots$. Для краткости мы будем называть $E_{n}$ канторовым множеством, а не “канторовым множеством генерации $n$ ", что было бы более правильно.

6.1. Емкость $\gamma_{s,+}$ канторовых множеств. Толсе принадлежит следующий результат [116; теорема 3.1].

ТеОрема 6.1. Пусть $\mu$ - вероятностная мера на $E_{n}$, равномерно распределенная на каждом кубе $E_{n, k}$ с $\mu\left(E_{n, k}\right)=2^{-d n}$. Пусть, далее, $0<s<d u$ $(2+\delta) \sigma_{j+1} \leqslant \sigma_{j}, j=0, \ldots, n-1$, где $\delta>0$. Тогда

$$
\left\|R^{s} \mu\right\|_{L^{2}(\mu)} \approx\left[\sum_{j=1}^{n} \theta_{j}^{2}\right]^{1 / 2}, \quad \theta_{j}=\frac{2^{-d j}}{\sigma_{j}^{s}} .
$$

С использованием методов из [59] отсюда выводится оценка

$$
\gamma_{s,+}\left(E_{n}\right) \approx\left[\sum_{j=1}^{n} \theta_{j}^{2}\right]^{-1 / 2}, \quad \theta_{j}=\frac{2^{-d j}}{\sigma_{j}^{s}} .
$$

Теорема 6.1 обобщает и улучшает серию предшествующих результатов. При $d=2, s=1$ (т. е. для емкости $\gamma_{+}$) и $\delta>1$ эта оценка была получена в [33] и независимо в диссертации Толсы (см. [109]). Соответствующие неравенства для $\gamma$ установлены в [60] (до теоремы Толсы о сравнимости $\gamma$ и $\gamma_{+}$). Случай липшицевой гармонической емкости (т. е. $d \geqslant 2, s=d-1$ ) рассмотрен в [59] при дополнительном условии $\theta_{j+1} \leqslant \theta_{j}$. Заметим, что условие $(2+\delta) \sigma_{j+1} \leqslant \sigma_{j}, \delta>0$, означающее разделенность кубов $E_{j, k}$, существенно используется в доказательстве всех указанных выше результатов. В дальнейшем мы применим (6.1), (6.2) 
для иллюстрации точности оценок емкости в терминах обхвата по Хаусдорфу. Мы увидим, что условие $\delta>0$ приводит к дополнительному ограничению на измеряющую функцию $h$, что влияет на общность утверждений. Чтобы избавиться от этого условия, в [36] модифицирована конструкция канторова множества. Для любой последовательности чисел $\sigma_{1}, \ldots, \sigma_{n}$ со свойством $2 \sigma_{j+1} \leqslant \sigma_{j}$ построена вспомогательная "регуляризованная" последовательность $\ell_{1}, \ldots, \ell_{n}$ такая, что $2 \ell_{j+1} \leqslant \ell_{j}$ и $\sigma_{j} \approx \ell_{j}, j=0, \ldots, n$, с константами сравнения, зависящими только от $s$ и $d$. Далее строится канторово множество, состоящее из $2^{n d}$ кубов со стороной $\ell_{n}$. Отличие состоит лишь в том, что теперь кубы генерации $j+1$ не обязательно расположены в углах кубов $j$-й генерации. Мы не приводим здесь конструкцию этого множества, отсылая читателя к статье [36]. Такое "регуляризованное" множество также обозначим через $E_{n}$. Для него оказываются справедливыми оценки (6.1), (6.2); применение этого множества не менее удобно, чем применение "углового" канторова множества. В [36] получена и оценка нормы оператора по мере $\mu$ из теоремы 6.1:

$$
\left|R_{\mu}^{s}\right| \approx\left[\sum_{j=1}^{n} \theta_{j}^{2}\right]^{1 / 2}
$$

Показано также, что $R^{s} \mu(x)$ принимает "большие" значения на "большой" порции множества $E_{n}$ :

$$
\mu\left\{x:\left|R^{s} \mu(x)\right|^{2}>c \sum_{j=1}^{n} \theta_{j}^{2}\right\}>\delta_{0}, \quad \delta_{0}=\delta_{0}(d, s)>0 .
$$

Ясно, что отсюда следует оценка снизу в (6.1), а значит, и в (6.3). Оценка сверху в (6.3) (и, следовательно, в (6.1)) вытекает из (5.2) (см. [34]). Оценка снизу в (6.2) легко следует из оценки сверху в (6.1). Таким образом, наиболее трудной частью (6.2) является доказательство оценки сверху. Оно проводится в [36] по той же схеме, что и в [59], и существенно опирается на $T(b)$-теорему Криста [18]. В [36] рассмотрен и предельный случай оценки (6.2) для множества $E:=\bigcap_{n=1}^{\infty} E_{n}$. В этом случае сумма в (6.2) берется от 0 до $\infty$.

6.2. Связь с нелинейной емкостью. В [116; §2] показано, что

$$
\dot{C}_{2(d-s) / 3,3 / 2}\left(E_{n}\right) \approx\left[\sum_{j=1}^{n} \theta_{j}^{2}\right]^{-1 / 2} .
$$

Таким образом, для канторовых множеств $E_{n}$ соотношение (5.5) имеет место при всех $s \in(0, d)$. Это подкрепляет гипотезу, что указанное соотношение справедливо для любых компактов при всех нецелых $s \in(0, d)$.

6.3. Точность оценки (5.12). Справедливо следующее утверждение [36; предложение 7.2].

ПРеДЛОЖЕНИЕ 6.2. Для любых $\eta>0, P>0 u N \geqslant N_{0}(d, s)$ cуществует мера $\nu$, состоящая из $N$ равных положительных зарядов и такая, что $\|\nu\|=\eta$ 
$u$

$$
\mathbf{M}>c(d, s) \frac{\|\nu\|}{P}\left[\int_{h^{-1}(C \mathbf{M} / N)}^{h^{-1}(\mathbf{M})}\left(\frac{h(t)}{t^{s}}\right)^{2} \frac{d t}{t}\right]^{1 / 2}, \quad C=C(d, s) \geqslant 1
$$

для любого числа $\mathbf{M} \geqslant M_{h}\left(\mathscr{Z}^{*}(\nu, P)\right)$.

Интересно, что заряды меры $\nu$, на которой достигается оценка (5.12), расположены в центрах кубов $E_{n, k}$, образующих соответствующее канторово множество (можно считать, что $N=2^{d n}$ ). Существование экстремальной меры было доказано в [34] с использованием вероятностных аргументов. Но конкретное множество представлено не было. Двумерный случай был ранее рассмотрен в [32], [33].

6.4. Точность оценки (5.9). В [36; предложение 7.3] доказано, что для любых $s, d$, для которых $0<s<d$, и для любой измеряющей функции $h$ существуют постоянная $C=C(d, s)$ и компакт $E$ такие, что $M_{h}(E)>0$ и

$$
\gamma_{s,+}(E) \leqslant C M_{h}(E)\left[\int_{0}^{t_{2}}\left(\frac{h(t)}{t^{s}}\right)^{2} \frac{d t}{t}\right]^{-1 / 2}, \quad \text { где } \quad h\left(t_{2}\right)=M_{h}(E) .
$$

Как следствие получается и неулучшаемость оценки (5.10) (с точностью до постоянных $c, C$ ).

6.5. Неулучшаемость следствия 5.4. Если интеграл в (5.8) расходится, то для любого $s \in(0, d)$ найдется канторово множество $E$ такое, что $M_{h}(E)>0$, но $\gamma_{s,+}(E)=0\left[36 ;\right.$ следствие 7.5]. Предполагается, что функция $h(r) / r^{d}$ не возрастает.

В указанных выше применениях связь между заданной функцией и соответствующим канторовым множеством реализуется равенством $h\left(\sigma_{j}\right)=2^{-d j} h\left(\sigma_{0}\right)$, $j=1, \ldots, n$. Поэтому дополнительные предположения относительно $\left\{\sigma_{j}\right\}$ влекут те или иные ограничения на $h$. В частности, наше естественное предположение о невозрастании функции $h(r) / r^{d}$ не гарантирует свойства $(2+\delta) \sigma_{j+1} \leqslant$ $\sigma_{j}, \delta>0$; оно требует дополнительного условия на $h$ (например, что $h(r) / r^{(d-\varepsilon)}$, $\varepsilon>0$, не возрастает).

6.6. Длина Фавара канторовых множеств. В то время как емкости K3 канторовых множеств имеют красивую оценку (6.2), подобный результат для длины Фавара (см. раздел 2) отсутствует. Но такая оценка представляла бы несомненный интерес. В частности, плоские канторовы множества $E=\bigcap_{j=1}^{\infty} E_{j}$ бесконечной длины являются естественными и простейшими объектами для тестирования гипотезы

$$
\gamma(E)=0 \quad \Longrightarrow \quad \operatorname{Fav}(E)=0
$$

В разделе 2 уже отмечалось, что обратная импликация, вообще говоря, неверна. Но будет ли она верна на канторовых множествах (пример Джонса-Мюраи [52] имеет гораздо более сложную структуру)? Отрицательный ответ также представлял бы интерес, так как давал бы более простой контрпример, чем в [52]. (Заметим, что $H^{1}(E)=\infty$ тогда и только тогда, когда $\lim _{j \rightarrow \infty} 2^{d j} \sigma_{j}=$ 
œ.) Учитывая (6.2), вопрос можно сформулировать так: имеет ли место импликация

$$
\sum \theta_{j}^{2}=\infty \quad \Longrightarrow \operatorname{Fav}(E)=0
$$

или обратная к ней для канторова множества $E$ ? В этом направлении нам известен только следующий результат ([37], см. также [67]):

$$
\operatorname{Fav}(E)=0 \quad \Longrightarrow \quad \sum \theta_{j}=\infty
$$

Обозначим $\delta$-окрестность множества $G$ через $\widetilde{G}_{\delta}$. Пусть $E^{1 / 4}-$ плоское угловое канторово множество, порожденное последовательностью $\sigma_{j}=(1 / 4)^{j}$, $j=0,1,2, \ldots$. Заметим, что $E^{1 / 4}$ - простейший пример совершенно неспрямляемого множества и $\operatorname{Fav}\left(E^{1 / 4}\right)=0$. Чтобы изучить свойства длины Фавара и развить технику ее оценивания, в ряде работ рассматривалась задача о нахождении оценок для $\operatorname{Fav}\left(\widetilde{E}_{\delta}^{1 / 4}\right)$ и, в частности, об определении скорости исчезновения этой величины при $\delta \rightarrow 0$. Маттила [67] доказал, что

$$
\operatorname{Fav}\left(\widetilde{E}_{\delta}^{1 / 4}\right) \geqslant \frac{c}{\log (1 / \delta)}
$$

Бейтман и Вольберг [6] улучшили этот результат:

$$
\operatorname{Fav}\left(\widetilde{E}_{\delta}^{1 / 4}\right) \geqslant \frac{c \log \log (1 / \delta)}{\log (1 / \delta)} .
$$

Вопрос о точности этой оценки остается открытым.

Оценки сверху, полученные в [95], [103], используют понятие $\log _{*} a-$ число логарифмов в цепочке $\log \log \ldots \log a, a>1$, делающее эту цепочку меньше или равной 1. Так, в [95] показано, что

$$
\operatorname{Fav}\left(\widetilde{E}_{\delta}^{1 / 4}\right) \leqslant C e^{-c \log _{*}(1 / \delta)} .
$$

В [83] эта оценка существенно улучшена и доведена до степенного убывания:

$$
\operatorname{Fav}\left(\widetilde{E}_{\delta}^{1 / 4}\right) \leqslant C_{\tau}\left(\log \frac{1}{\delta}\right)^{-1 / 6+\tau}, \quad \tau>0 .
$$

Неизвестно, какой показатель является точным. В частности, можно ли заменить его на $1-\varepsilon$, где $\varepsilon>0$ ? Для такого улучшения нужны методы, отличные от использованных в [83].

Бонд и Вольберг [8], [9] рассмотрели аналогичную задачу для самоподобного "треугольного" канторова множества $\Delta^{1 / 3}$, при построении которого $j$-я генерация состоит из $3^{j}$ правильных треугольников со стороной $(1 / 3)^{j}, j=$ $0,1, \ldots$, расположенных в углах треугольников предыдущей генерации (при $j \geqslant 1)$. В [8], [9] доказано, что: 1) $\operatorname{Fav}\left(\widetilde{\Delta}_{\delta}^{1 / 3}\right) \leqslant B(\log (1 / \delta))^{-b}$ с некоторыми положительными постоянными $b, B ; 2)$ для произвольного самоподобного канторова множества $E$ хаусдорфовой размерности 1 выполнено неравенство $\operatorname{Fav}\left(\widetilde{E}_{\delta}\right) \leqslant C e^{-c \sqrt{\log \log (1 / \delta)}}$. Для многих (но не всех) самоподобных канторовых множеств размерности 1 последнее неравенство улучшено в [7]. Пока эта оценка 
имеет "степенной тип" $\leqslant C(\log (1 / \delta))^{-p}$ только в особых случаях (мы не приводим описание таких множеств, отсылая читателя к работе [7]). Неизвестно, справедлива ли такая оценка (пусть даже с малым $p$ ) для всех самоподобных канторовых множеств размерности 1.

\section{7. Феномен нецелых $s$}

В разделах 7-9 мы возвращаемся к основной теме данного обзора - связи между аналитическими свойствами интегральных преобразований и операторов и геометрическими свойствами носителя меры. Пусть $K(x, y)$ - ядро КЗ порядка $s \in(0, d]$. Рассмотрим положительную конечную меру $\mu$ в $\mathbb{R}^{d}$. Если меpa $\mu$ "размазана", т. е. $\mu(B(x, r)) \leqslant C r^{s+\varepsilon}, \varepsilon>0$, то оператор $T: L^{2}(\mu) \rightarrow L^{2}(\mu)$ и преобразование $T \mathbf{1}(x)$ с ядром $K$ ограничены (это видно, например, из того, что ядро порядка $s$ даже не сингулярно по отношению к мере порядка $s+\varepsilon$ ). С другой стороны, из ограниченности преобразования Рисса $R^{s} \mu(x)$ в $L^{\infty}\left(\mathbb{R}^{d}\right)$ следует, что $\mu(B(x, r)) \leqslant C r^{s}$ (см. замечание после равенства (4.2)). Поэтому интересной будет ситуация, когда порядок роста меры совпадает с порядком ядра. Предположим дополнительно, что $0<H^{s}(\operatorname{supp} \mu)<\infty$, и рассмотрим ядро Рисса $x /|x|^{s+1}$. Мы будем говорить, что преобразование Рисса $R^{s} \mu(x)$ ограничено, если $\left\|R^{s} \mu\right\|_{L^{\infty}\left(\mathbb{R}^{d}\right)}<\infty$.

Для целых $s$ нетрудно построить меру, для которой преобразование $R^{s} \mu(x)$ существует (в смысле главного значения) и ограничено всюду в $\mathbb{R}^{d}$. Действительно, возьмем $s$-мерный шар $B \subset \mathbb{R}^{d}$, выберем $C^{\infty}$-функцию $\varphi$ с носителем на $B$ и положим $\mu=\varphi m_{s}$, где $m_{s}-s$-мерная мера Лебега. Очевидно, существование и ограниченность преобразования $R^{s} \mu(x)$ возникает здесь за счет взаимного сокращения вкладов тех элементов меры $\mu$, которые симметричны относительно точки $x$ и близки к $x$. Ясно, что “хорошая" геометрия носителя меры играет здесь важную роль.

Если $s$ нецелое, то такая конструкция не проходит: носитель $E$ меры, для которого $0<H^{s}(E)<\infty$, имеет фрактальную структуру с обилием "крайних" точек $x$, относительно которых мера "не уравновешена". Этим и объясняется феномен нецелых $s$, о котором пойдет речь в данном разделе.

7.1. Ограниченность преобразований Рисса. Одна из наиболее известных и интригующих задач излагаемой теории - доказать следующее утверждение (насколько нам известно, никто не верит, что его можно опровергнуть).

ГиПотеЗА 7.1. Пусть $s \in(0, d) \backslash \mathbb{N}$, и пусть положительная конечная борелевская мера $\mu$ такова, что $H^{s}(\operatorname{supp} \mu)<\infty$. Тогда $\left\|R^{s} \mu\right\|_{L^{\infty}\left(\mathbb{R}^{d}\right)}=\infty$.

Другими словами, если нещелое $s$ принадлежит интервалу $(0, d)$ и компакт $E$ удовлетворяет условию $H^{s}(E)<\infty$, mо $\gamma_{s,+}(E)=0$.

Полезна еще одна эквивалентная формулировка: если оператор $R_{\mu}^{s}, 0<s \leqslant$ $d$, ограничен в $L^{2}(\mu)$ u $H^{s}(\operatorname{supp} \mu)<\infty$, то s целое.

При $0<s<1$ эту гипотезу доказала Прат [96], существенно используя понятие кривизны меры. Мы уже не раз с сожалением констатировали, что при $s>1$ этот инструмент отсутствует. Вихтила [124] доказала гипотезу 7.1 для 
всех нецелых $s \in(0, d)$, но при следующем дополнительном условии: нижняя $s$-плотность $\Theta_{*}(x)$ меры $\mu$ положительна для $\mu$-почти всех $x \in \operatorname{supp} \mu$; здесь

$$
\Theta_{*}(x):=\liminf _{r \rightarrow 0} \frac{\mu(B(x, r))}{r^{s}} .
$$

Доказательство в [124] основано на использовании касательной меры. Этот метод также не работает в общем случае, поскольку при отсутствии каких-либо предположений относительно нижней плотности касательная мера уже не обязательно $s$-мерна. С другой стороны, в [124] доказано больше, чем сформулировано. В точности те же аргументы доказывают нужное утверждение для любой конечной меры $\mu$, для которой $\mu\left(\left\{x: \Theta_{*}(x)>0\right\}\right)>0$. Таким образом, чтобы доказать гипотезу 7.1, достаточно рассмотреть меры, для которых $\Theta_{*}(x)=0$ при $\mu$-почти всех $x \in \mathbb{R}^{d}$. Такие меры называются тотально нерегулярными. Заметим, что положительные тотально нерегулярные меры существуют только при $s<d$. Прежде чем заняться такими мерами, отметим еще один случай, в котором гипотеза 7.1 доказана, - а именно, когда носитель $E$ меры $\mu$ является канторовым множеством (см. раздел 6) с $H^{s}(E)<\infty$. В этом случае $\liminf \operatorname{in}_{j \rightarrow \infty} 2^{d j} \sigma_{j}^{s}<\infty$, и в силу (6.2) выполнено равенство $\gamma_{s,+}(E)=0$. Но при доказательстве соотношения (6.2) существенно используется "правильная" структура канторова множества. Поэтому метод, примененный в следующей теореме из [35], совершенно иной, чем в работах всех предшествующих авторов.

ТЕОРема 7.2. Пусть $s \in[d-1, d)$ u $\mu$ - положительная конечная тотально нерегулярная борелевская мера в $\mathbb{R}^{d}, d \geqslant 2$, такая, что $H^{s}(\operatorname{supp} \mu)<\infty$. Тогда $\left\|R^{s} \mu\right\|_{L^{\infty}\left(\mathbb{R}^{d}\right)}=\infty$ и оператор $R_{\mu}^{s}$ неограничен в $L^{2}(\mu)$.

Хотя рассуждение в [35] проводится для случая $d=2$, оно без изменений переносится на общий случай $d \geqslant 2$ (это отмечено в [35]). Таким образом, теорема 7.2, будучи объединенной с результатами Прат и Вихтилы, завершает доказательство гипотезы 7.1 при $s \in(0,1) \cup(d-1, d)$, "откусывая" и правый участок интервала $(0, d)$. Тем самым в случае $d=2$ гипотеза 7.1 оказывается полностью доказанной.

Кратко опишем применяемый в [35] метод, а также основное препятствие для его распространения на значения $s$, меньшие $d-1$. Прежде всего заметим, что достаточно рассмотреть случай $\mu \in \Sigma_{s}$ (см. абзац после (4.2)). Следовательно, вместо неограниченности преобразования Рисса можно доказывать неограниченность максимального оператора $R_{\mu}^{\sharp}$ в $L^{2}(\mu)$. Действительно, неограниченность оператора $R_{\mu}^{\sharp}$ равносильна неограниченности оператора $R_{\mu}^{s}$ (теорема 3.6). Это означает, что $\gamma_{\text {ор }}(E)=0$ для любого компакта $E$ такого, что $H^{s}(E)<\infty$. Но тогда и $\gamma_{s,+}(E)=0$ - см. (4.8)-(4.10).

Предположим противное, т. е. найдется конечная тотально нерегулярная мера $\mu \in \Sigma_{s}$ такая, что $H^{s}(\operatorname{supp} \mu)<\infty$ и оператор $R_{\mu}^{\sharp}$ ограничен в $L^{2}(\mu)$. Как уже отмечалось в конце раздела 4, переход к операторной норме удобен тем, что при удалении порции меры ограниченность оператора сохраняется и норма не возрастает. Поэтому можно удалить часть (но не очень большую) меры, придав оставшейся части структуру, аналогичную структуре канторова множества (разумеется, далеко не столь регулярную, как в разделе 6). Полученную 
меру обозначим $\mu^{\prime}$. Такая структура позволяет разбить преобразование Рисса на сумму интегралов $R^{(n)} \mu^{\prime}(x)$ - сужений преобразования $R^{s} \mu^{\prime}$ на соответствующие уровни. Далее доказывается, что:

(i) модуль $\left|\sum_{n=0}^{N-1} R^{(n)} \mu^{\prime}(x)\right|$ ограничен сверху величиной, не зависящей от $x$ и $N$;

(ii) функции $R^{(n)} \mu^{\prime}(x)$ "почти ортогональны" в $L^{2}\left(\mu^{\prime}\right)$;

(iii) при достаточно больших $N$ нормы $\left\|R^{(n)} \mu^{\prime}\right\|_{L^{2}\left(\mu^{\prime}\right)}$ настолько велики, что их оценки снизу и (ii) противоречат оценке (i).

Наиболее сложным пунктом этой программы оказалось доказательство свойства (iii). Оно, в свою очередь, разбивается на несколько этапов, включающих переход к еще более "хорошим" мерам с $C^{\infty}$-плотностью относительно лебеговой меры $m_{d}$, решение определенной экстремальной задачи, а также доказательство и применение принципа максимума для сопряженного к $R^{s}$ преобразования Рисса $R^{*} \eta=-\sum_{j}^{d} R_{j}^{s} \eta_{j}$; здесь $\eta=\left(\eta_{1}, \ldots, \eta_{d}\right)$ - векторнозначная конечная мера с $C^{\infty}$-плотностью и $R_{j}^{s} \eta_{j}-j$-я координата преобразования $R^{s} \eta_{j}$. Принцип максимума состоит в следующем:

$$
\max _{\mathbb{R}^{d}} R^{*} \eta=\max _{\operatorname{supp} \eta} R^{*} \eta
$$

в предположении, что левая часть положительна и $s \geqslant d-1$. В случае $s=d-1$ это обычный принцип максимума для гармонических функций. При $s>d-1$ он выводится из формулы

$$
p(x)=\sigma \int_{\mathbb{R}^{d}} \frac{u(x+y)-u(x)}{|y|^{2 d-s+1}} d y, \quad s>d-1,
$$

восстанавливающей плотность $p$ действительного заряда $\nu=p(y) d y$ с компактным носителем по его потенциалу

$$
u(x)=\frac{1}{1-s} \int_{\mathbb{R}^{d}} \frac{d \nu(y)}{|x-y|^{s-1}} ;
$$

здесь $\sigma$ - постоянная, зависящая от $d$ и $s$. Переход от $R^{*} \eta$ к $u$ осуществляется равенствами

$$
\begin{gathered}
R_{j}^{s} \eta_{j}(x)=\frac{\partial}{\partial x_{j}}\left(\frac{1}{1-s} \int_{\mathbb{R}^{d}} \frac{d \eta_{j}(y)}{|x-y|^{s-1}}\right)=\frac{1}{1-s} \int_{\mathbb{R}^{d}} \frac{p_{j}(y) d y}{|x-y|^{s-1}}, \\
p(y)=-\sum_{j}^{d} p_{j}(y),
\end{gathered}
$$

где

$$
d \eta_{j}(y)=\rho_{j}(y) d y, \quad p_{j}(y)=\frac{\partial \rho_{j}(y)}{\partial y_{j}} .
$$

Но при $s<d-1$ формула восстановления (7.1) меняет вид: она становится более сложной и включает лапласианы $\Delta u(x)$. Получить для них нужную оценку не представляется возможным. 
Какая-либо форма принципа максимума с $s<d-1$ (не обязательно такая, как указана выше) была бы исключительно полезной для доказательства гипотезы 7.1. Приведем одну из возможных формулировок в виде гипотезы, представляющей и самостоятельный интерес.

ГиПотезА 7.3. Пусть $\xi-$ конечная знакопеременная мера (заряд) в $\mathbb{R}^{d}$ с компактным носителем и $C^{\infty}$-плотностью относительно лебеговой меpъь $m_{d}$. Тогда

$$
\left|R^{s} \xi(x)\right|<C \max _{y \in \operatorname{supp} \xi}\left|R^{s} \xi(y)\right|, \quad \forall x \in \mathbb{R}^{d}, \quad 0<s \leqslant d, \quad C=C(d, s) .
$$

Это утверждение не должно зависеть от того, будет $s$ целым или нет.

При $d-1 \leqslant s \leqslant d$ гипотеза 7.3 следует из неравенства

$$
\left|R_{j}^{s} \xi(x)\right|<\max _{y \in \operatorname{supp} \xi}\left|R_{j}^{s} \xi(y)\right|, \quad \forall x \in \mathbb{R}^{d}, \quad d-1 \leqslant s \leqslant d, \quad j=1, \ldots, d,
$$

которое при $s>d-1$ вытекает из (7.1). При малых $s$ последнее неравенство, вообще говоря, уже не имеет места для всех $j=1, \ldots, d$.

Совсем недавно Джей и Назаров [48] получили новое красивое доказательство теоремы 7.2, основанное на обнаруженной ими связи между изучаемой задачей и свойством "неотражаемости" меры. Описательно говоря, мера $\mu$ называется неотражающей (reflectionless) для оператора $T$, если потенциал $T \mu(x)$ обращается в нуль (в слабом смысле) на носителе меры. Мы отсылаем читателя к работе [48] за точными формулировками, библиографией, а также за интересными фактами о геометрических свойствах неотражающих мер и об их связи с другими задачами гармонического анализа.

7.2. Количественная версия гипотезы 7.1. Мы уже упоминали, что для канторовых множеств гипотеза 7.1 следует из соотношения (6.2). На самом деле (6.2) содержит гораздо большую информацию, чем та, которую мы использовали. Для "полноценного" канторова множества $E=\bigcap_{n=1}^{\infty} E_{n}$ и вероятностной меры - слабого предела мер из теоремы 6.1 - это неравенство можно переписать в виде

$$
\gamma_{s,+}(E) \approx \sup _{x \in \operatorname{supp} \mu}\left[W^{\mu}(x)\right]^{-1 / 2}
$$

где $W^{\mu}(x)$ - потенциал Вольфа (см. (5.2)). Тесная связь между емкостью $\gamma_{s,+}$ произвольных компактов и потенциалом Вольфа видна также из неравенства (5.3).

Пусть функция $\Phi:[0, \infty) \rightarrow[0, \infty)$ такова, что $\Phi(0)=0, \Phi$ непрерывна и строго возрастает, и пусть найдутся положительные числа $\sigma$ и $b$ такие, что $\Phi(t) / t^{\sigma}$ не убывает на $(0, b)$. Следуя Джею, Назарову и Вольбергу [49], определим потенциал Вольфа, ассоциированный с $\Phi$, равенством

$$
\mathscr{W}_{\Phi, s}^{\mu}(x)=\int_{0}^{\infty} \Phi\left(\frac{\mu(B(x, r))}{r^{s}}\right) \frac{d r}{r} .
$$


Очевидно, что $\mathscr{W}_{\Phi, s}^{\mu}(x)=W^{\mu}(x)$ при $\Phi(t)=t^{2}$. Определим емкость компакта $E \subset \mathbb{R}^{d}$, ассоциированную с функцией $\Phi:$

$$
\operatorname{cap}_{\Phi, s}(E)=\sup \left\{\|\mu\|: \operatorname{supp} \mu \subset E, \mathscr{W}_{\Phi, s}^{\mu}(x) \leqslant 1 \text { для всех } x \in \mathbb{R}^{d}\right\} .
$$

В [49] установлен ряд свойств емкости $\operatorname{cap}_{\Phi, s}$ и потенциала $\mathscr{W}_{\Phi, s}^{\mu}$.

ГиПотезА 7.4. Пусть $d \geqslant 2,0<s<d, s \notin \mathbb{N}$. Тогда для любого компакта $E \subset \mathbb{R}^{d}$

$$
\gamma_{s,+}(E) \approx \operatorname{cap}_{\Phi, s}(E), \quad \Phi(t)=t^{2},
$$

с константами сравнения, зависящими только от $d u s$.

Несложно доказать [49; лемма 7.2], что сар ${ }_{\Phi, s}(E)=0$ для любого компакта $E \subset \mathbb{R}^{d}$ такого, что $H^{s}(E)<\infty$. Поэтому гипотеза 7.4 содержит гипотезу 7.1 . По существу, гипотеза 7.4 доказана (хотя и сформулирована по-другому) в [57] для $0<s<1$. Аналог для случая $s=0$ рассмотрен в [1]. Более того, в силу (5.3) получаем

$$
\gamma_{s,+}(E) \geqslant c(d, s) \cdot \operatorname{cap}_{\Phi, s}(E), \quad \Phi(t)=t^{2}, \quad 0<s<d .
$$

Здесь не требуется, чтобы $s$ было целым. Но обратное неравенство для целых $s$ неверно. Например, s-мерный шар $B \subset \mathbb{R}^{d}$ имеет положительную емкость $\gamma_{s,+}$, но $\operatorname{cap}_{\Phi, s}(B)=0$.

Во всех работах, указанных выше, существенно использовался метод кривизны меры. В [49] впервые получена оценка сверху емкости $\gamma_{s,+}$ через потенциал Вольфа для значений $s$, при которых метод кривизны не применим.

ПредлОЖЕНИЕ 7.5. Пустъ $d-1<s<d$. Найдутся положителъные постоянные $\beta, C$, зависящие от $d, s$ и такие, что для любого компакта $E \subset \mathbb{R}^{d}$

$$
\gamma_{s,+}(E) \leqslant C \operatorname{cap}_{\Phi, s}(E), \quad \Phi(t)=e^{-1 / t^{\beta}} .
$$

Конечно, это меньше, чем хотелось бы. Но повторим, что это первый общий результат для $s>1$, не использующий кривизну. В доказательстве применяется метод из теоремы 7.2 [35] (отсюда и ограничение $d-1<s<d$ ), а также деликатное рассуждение, позволившее снабдить идеи Вихтилы [124] количественными оценками.

7.3. Существование главных значений и другие свойства преобразований Рисса, возможные лишь при целых $s$. Еще одним "хорошим" аналитическим свойством преобразования Рисса $R^{s} \mu$, которое может выполняться лишь при целых $s$, является существование его главных значений $\mu$-почти всюду. Более того, это условие настолько сильное, что при дополнительных предположениях относительно $\mu$ несет важную информацию о структуре носителя меры. Обозначим через $\Theta^{*}$ верхнюю $s$-плотность меры $\mu$ :

$$
\Theta^{*}(x):=\limsup _{r \rightarrow 0} \frac{\mu(B(x, r))}{r^{s}} .
$$

Теорема 7.6. Пусть $0 \leqslant s \leqslant d$, пусть положительная конечная мера Радона $\mu$ в $\mathbb{R}^{d}$ такова, что $0<\Theta^{*}(x)<\infty$ u $\lim _{\varepsilon \rightarrow 0} R_{\varepsilon}^{s} \mu(x)$ существует для всех $x$ из множества положительной $\mu$-меры. Тогда s целое. 
Это утверждение доказали Руиз де Вилла и Толса [101; теорема 1.1]. Оно усиливает результат Маттилы и Прейса [72; теорема 4.5], в котором дополнительно предполагалось, что $\Theta_{*}(x)>0$ и главные значения существуют $\mu$-почти всюду в $\mathbb{R}^{d}$.

В работе Вихтилы [124; теорема 5] условие на плотность меры тоже более сильное, чем в теореме 7.6: $0<\Theta_{*}(x) \leqslant \Theta^{*}(x)<\infty$ для $\mu$-почти всех $x \in \operatorname{supp} \mu$. При этом существование главных значений преобразования Рисса заменено предположением $R^{\#} \mu(x)<\infty$ для $\mu$-почти всех $x \in \mathbb{R}^{d}$. Из этих двух условий в [124] выводится, что $s \in \mathbb{Z}$. Доказательство в [124] основано на использовании касательных мер. Мы уже упоминали, что эти аргументы не работают при $\Theta_{*}(x)=0$. Для значений $s \in[0,1] \cup[d-1, d]$ указанный результат Вихтилы может быть усилен.

ПРеДЛОЖЕНИЕ 7.7. Пусть $s \in[0,1] \cup[d-1, d]$, и пусть положителъная борелевская мера $\mu$ в $\mathbb{R}^{d}$ такова, что $0<\Theta^{*}(x)<\infty$ и $R^{\#} \mu(x)<\infty$ для $\mu$-почти всех $x \in \mathbb{R}^{d}$. Тогда s иелое.

Предложение 7.7 непосредственно вытекает из следующего утверждения.

ПреДЛОЖЕНИЕ 7.8. Пустъ $s \in(0,1) \cup(d-1, d)$, и пустъ положительная борелевская мера $\mu$ в $\mathbb{R}^{d}$ такова, что $0<\Theta^{*}(x)<\infty$ для $\mu$-почти всех $x \in \mathbb{R}^{d}$. Тогда множество $G:=\left\{x \in \mathbb{R}^{d}: R^{\#} \mu(x)=\infty\right\}$ имеет положительную $\mu$-меру.

При $s \in(0,1)$ это утверждение было по существу доказано Прат [97; теорема 2] (хотя наша формулировка несколько сильнее). Доказательство в [97] основано на использовании аналога кривизны меры (см. раздел 5), а также на результате Назарова-Треиля-Вольберга, который мы сформулируем в усиленном виде по сравнению с версией, приведенной в [97; теорема 4].

ТЕорема 7.9. Пусть $0<s<d$, и пусть положительная мера $\mu$ в $\mathbb{R}^{d}$ maкова, ито $\Theta^{*}(x)<\infty$ и $R^{\#} \mu(x)<\infty$ для $\mu$-почти всех $x \in \mathbb{R}^{d}$. Тогда найдется такое множество $F$, что $\mu(F)>0$ и оператор $R_{\mu \mid F}^{s}$ ограничен в $L^{2}(\mu \mid F)$.

В случае оператора Коши (т. е. при $d=2, s=1$ ) теорема 7.9 является следствием теоремы 3 из работы [89; с. 65], в которой получена также оценка нормы оператора. Доказательство этой теоремы 3 не использует кривизны меры и с очевидными модификациями проходит для $0<s<d, d \geqslant 2$.

ДоКАЗАТЕЛЬСТВо ПРЕДЛОЖЕНИЯ 7.8. Рассуждая от противного, предположим, что $R^{\#} \mu(x)<\infty \mu$-почти всюду. Пусть $F$ - множество из теоремы 7.9 и $F_{i}:=\left\{x \in F: \Theta^{*}(x)>2^{-i}\right\}, i=0,1, \ldots$. Можно предполагать, что $\mu(F)<\infty$. Так как $\Theta^{*}(x)>0 \quad \mu$-почти всюду, то найдется такой индекс $k$, для которого $\mu\left(F_{k}\right)>0$. Нетрудно показать, что $H^{s}\left(F_{k}\right)<\infty$. Действительно, зафиксируем произвольное $\delta>0$ и для каждой точки $x \in F_{k}$ выберем шар $B(x, r(x))$ такой, что $r(x)<\delta$ и $\mu(B(x, r(x)))>(1 / 2) 2^{-k} r(x)^{s}$. Из полученного покрытия множества $F_{k}$ по лемме Безиковича выделим не более чем счетное подпокрытие $\left\{B\left(x_{j}, r\left(x_{j}\right)\right)\right\}$ конечной кратности $A(d)$. Имеем

$$
\sum_{j} r\left(x_{j}\right)^{s}<2^{k+1} \sum_{j} \mu\left(B\left(x_{j}, r\left(x_{j}\right)\right)\right)<2^{k+1} A(d) \mu\left(F_{k}\right) .
$$


Поскольку правая часть не зависит от $\delta$, то $H^{s}\left(F_{k}\right)<\infty$. Далее, в силу теоремы 7.9 оператор $R_{\mu \mid F_{k}}^{s}$ ограничен в $L^{2}\left(\mu \mid F_{k}\right)$. Значит, емкость $\gamma_{\text {ор }}\left(F_{k}\right)$, а с ней и $\gamma_{s,+}\left(F_{k}\right)$, положительны (см. (4.8)-(4.10)). Это противоречит гипотезе 7.1, которая при $s \in(0,1) \cup(d-1, d)$ является теоремой. Предложение доказано.

Другие условия, альтернативные существованию главных значений в теореме 7.6, рассмотрели Вольберг и Майборода [74]. Используя технику работы [101], они получили следующий результат. Положим

$$
R_{\varepsilon, \delta}^{s} \mu(x):=R_{\varepsilon}^{s} \mu(x)-R_{\delta}^{s} \mu(x), \quad 0<\varepsilon<\delta .
$$

Теорема 7.10. Пусть конечная мера Радона $\mu$ в $\mathbb{R}^{d}$ обладает следующими свойствами: $0<\Theta^{*}(x)<\infty$ для $\mu$-почти всех $x \in \mathbb{R}^{d}$ и при некотором $s \in(0, d]$ выполнено хотя бы одно из условий

$$
\begin{aligned}
& S \mu(x):=\left(\int_{0}^{\infty}\left|R_{t, 2 t}^{s} \mu(x)\right|^{2} \frac{d t}{t}\right)^{1 / 2}<\infty \quad \text { для } \mu \text {-почти всех } x \in \mathbb{R}^{d}, \\
& \lim _{\varepsilon \rightarrow 0} R_{\varepsilon, 2 \varepsilon}^{s} \mu(x)=0 \quad \text { для } \mu \text {-почти всех } x \in \mathbb{R}^{d} .
\end{aligned}
$$

Тогда s иелое.

Ясно, что условие (7.3) слабее условия о существовании главных значений в соответствующих точках.

\section{8. Существование главных значений в случае целых $s$}

В данном и следующем разделах мы увидим, что в случае целых $s$ "хорошие" аналитические свойства преобразований и операторов Рисса взаимосвязаны со свойствами меры и геометрической структурой ее носителя.

8.1. Определения. Мера $\mu$ называется s-регулярной в смысле Альфорса-Давида, или просто $s$-АД- или АД-регулярной, или однородной, если найдутся положительные постоянные $c, C$, для которых

$$
c r^{s} \leqslant \mu(B(x, r)) \leqslant C r^{s}, \quad \forall x \in \operatorname{supp} \mu, \quad 0<r \leqslant \operatorname{diam}(\operatorname{supp} \mu) .
$$

Множество $E$ называется АД-регулярным, если АД-регулярна мера $\mu=H^{s} \mid E$.

В дальнейшем через $n$ обозначается натуральное число.

Множество $E \subset \mathbb{R}^{d}$ называется $n$-спрямляемым, если найдутся липшицевы отображения $f_{i}: \mathbb{R}^{n} \rightarrow \mathbb{R}^{d}, i=1,2, \ldots$, такие, что

$$
H^{n}\left(E \backslash \bigcup_{i} f_{i}\left(\mathbb{R}^{n}\right)\right)=0
$$

Если множество $E$ является $H^{n}$-измеримым и $H^{n}(E)<\infty$, то в определении $n$-спрямляемости липшицевы образы $f_{i}\left(\mathbb{R}^{n}\right)$ можно заменить $n$-мерными $C^{1}$-подмногообразиями в $\mathbb{R}^{d}-$ см. [39; гл. 3]. Другими словами, $H^{n}$-измеримое множество $E$ с $H^{n}(E)<\infty$ является $n$-спрямляемым тогда и только тогда, когда с точностью до множества нулевой $H^{n}$-меры его можно покрыть не более 
чем счетным набором $n$-мерных $C^{1}$-подмногообразий. Заметим, что определение 1-спрямляемости, данное в конце раздела 2, эквивалентно определению $n$-спрямляемости при $n=1$, поскольку каждую спрямляемую кривую можно покрыть счетным набором графиков липшицевых функций плюс множеством нулевой $H^{1}$-меры [93; с. 12]. Ряд других важных свойств $n$-спрямляемых множеств имеется в [39], [63], [93].

Мера Радона $\mu$ в $\mathbb{R}^{d}$ называется $n$-спрямляемой, если она абсолютно непрерывна относительно $H^{n}$ и найдется $n$-спрямляемое борелевское множество $E$ такое, что $\mu\left(\mathbb{R}^{d} \backslash E\right)=0$.

Давид и Семмс [29] ввели понятие равномерно $n$-спрямляемого множества (задача, в связи с которой возникло это понятие, обсуждается в разделе 9). Одно из многих эквивалентных определений состоит в следующем. Множество $E \subset \mathbb{R}^{d}$ называется равномерно $n$-спрямляемым, если для любых $x \in E$ и $r \in(0, \operatorname{diam} E)$ найдется липшицево отображение $g$-мерного шара $B_{n}(0, r)$ в $\mathbb{R}^{d}$ такое, что

$$
g(0)=x \quad \text { и } \quad H^{n}\left(E \cap B(x, r) \cap g\left(B_{n}(0, r)\right)\right) \geqslant c r^{n},
$$

причем постоянная $c$ и липшицевы константы отображений $g$ не зависят от $x, r$. Согласно терминологии Давида-Семмса это означает, что $E$ содержит большие куски липшицевых образов пространства $\mathbb{R}^{n}$. Можно показать, что всякое равномерно $n$-спрямляемое множество $n$-спрямляемо, но обратное неверно - см. пример 8.7.

Аналогично определяются равномерно $n$-спрямляемые меры [115]: условие $H^{n}(\cdot) \geqslant c r^{n}$ заменяется неравенством

$$
\mu\left(B(x, r) \cap g\left(B_{n}(0, r)\right)\right) \geqslant c r^{n} .
$$

Очевидно, борелевское множество $E \subset \mathbb{R}^{d}$ равномерно $n$-спрямляемо, если мера $\mu=H^{n} \mid E$ равномерно $n$-спрямляема.

При $n=1$ равномерная 1-спрямляемость множества равносильна тому, что множество 1-АД-регулярно и является подмножеством 1-АД-регулярной кривой. Это следует из результатов Давида-Семмса [29; с. 22] и Джонса [51], [29; с. 19] при $n=1, d=2$ и Окикиолу [91] при $n=1, d \geqslant 2$. Важную роль здесь сыграли $\beta$-числа Питера Джонса, позволившие ему также установить тесную связь между ограниченностью оператора Коши и задачей о коммивояжере в следующей редакции: найти связное множество наименьшей длины, содержащее данное множество.

Но при $n>1$ такое описание равномерной $n$-спрямляемости уже не имеет места. Многочисленные свойства равномерно спрямляемых множеств приведены в [28], [29].

8.2. Существование главных значений и спрямляемость. Мы уже упоминали, что при дополнительных предположениях относительно меры существование главных значений преобразования Рисса (возможное, как мы видели, лишь при целых $s$ ) дает важную информацию о геометрии ее носителя.

Рассмотрим вначале достаточные условия существования главных значений. Маттила и Мельников [69] получили следующий результат. 
Теорема 8.1. Пусть $\nu$ - конечная комплексная борелевская мера в $\mathbb{C}$ и Г график липшицевой функиии. Тогда предел $\lim _{\varepsilon \rightarrow 0} \mathscr{C}_{\varepsilon} \nu(z)$, где

$$
\mathscr{C}_{\varepsilon} \nu(z):=\int_{|\xi-z|>\varepsilon}(\xi-z)^{-1} d \nu(\xi),
$$

существует и конечен почти всюду на Г относительно меры $H^{1}$.

Ясно, что вместо Г можно взять и счетное объединение графиков липшицевых функции. Поэтому кривую Г в теореме 8.1 можно заменить спрямляемой кривой либо 1-спрямляемым множеством. Чтобы доказать теорему 8.1, представим меру $\nu$ в виде $\nu=\nu_{0}+\sigma$, где $\nu_{0}$ абсолютно непрерывна, а $\sigma$ сингулярна относительно $H^{1} \mid \Gamma$. Для $\nu_{0}$ существование главных значений $H^{1}$-почти всюду на $Г$ известно - см. [11]. Основной этап рассуждения в случае $\sigma$ состоит в доказательстве оценки слабого типа для максимального преобразования Коши $\mathscr{C}^{\sharp} \nu$ на Г. Другое, более короткое доказательство этой оценки получено Вердерой [121].

Маттила и Прейс [72; с. 492] отметили, что аргументы Вердеры работают и в многомерном случае, если воспользоваться ограниченностью оператора $R_{H^{n}}^{n} \mid$ Г на графиках $\Gamma$ липшицевых функций, заданных на $n$-мерных плоскостях. Таким образом, справедливо следующее утверждение [72; с. 492]. Пусть E$n$-спрямляемое множество в $\mathbb{R}^{d}$ с $H^{n}(E)<\infty, 0<n \leqslant d$. Тогда преобразование Pисса $R^{n}\left(H^{n} \mid E\right)$ имеет главные значения $H^{n}$-почти всюду на $E$. В [72; с. 492] получено и обратное утверждение, которое мы обсудим ниже.

Заметим, что оценка слабого типа для максимального преобразования Рисса имеет место в весьма общей ситуации - см. теорему 3.6. Поэтому схему доказательства теоремы 8.1 можно применить и к преобразованию Рисса $R^{n} \nu$ по любой конечной борелевской мере $\nu$. Получается прямое обобщение теоремы 8.1: конечные главные значения преобразования $R^{n} \nu$ существуют $H^{n}$-почти всюду на любом $n$-спрямляемом множестве $E \subset \mathbb{R}^{d}$ (случай $n=d-1$ см. также в $[17$; теорема 1.5]).

Следующее усиление теоремы 8.1 принадлежит Толсе [106].

Теорема 8.2. Пусть $\mu$ - непреръвная (т.е. без атомов) положительная мера Радона в $\mathbb{C}$. Если оператор Коши $\mathscr{C}_{\mu}$ ограничен в $L^{2}(\mu)$, то для любой конечной комплексной меры Радона $\nu$ в $\mathbb{C}$ предел $\lim _{\varepsilon \rightarrow 0} \mathscr{C}_{\varepsilon} \nu(z)$ существует и конечен для $\mu$-почти всех $z \in \mathbb{C}$.

Теорема 8.1 сразу следует из теоремы 8.2, если положить $\mu=H^{1} \mid \Gamma$ и воспользоваться ограниченностью оператора Коши на графиках липшицевых функций. Этот классический результат Кальдерона (для малых констант) и Койфмана-Макинтоша-Мейера [19] развивался рядом авторов. Далеко идущие обобщения получены в [17] и [56].

Используя теорему 8.1, Толса [106] описал положительные меры Радона $\mu$ в $\mathbb{C}$, обладающие следующим свойством: для любой конечной комплексной меры Радона $\nu$ в $\mathbb{C}$ главные значения преобразования Коши $\mathscr{C} \nu(z)$ существуют $\mu$-почти всюду в $\mathbb{C}$.

В то же время неясно, как получить многомерное обобщение теоремы 8.2 , а именно следующее утверждение: если мера $\mu$ в $\mathbb{R}^{d}$ такова, что оператор $R_{\mu}^{n}$ 
ограничен в $L^{2}(\mu)$, то преобразование Рисса $R^{n} \nu$ имеет главные значения $\mu$-почти всюду в $\mathbb{R}^{d}$. Это неясно даже при $n=d-1$. По-видимому, такое утверждение верно. Но в случае $n=1, d=2$, рассмотренном Толсой, существенно используется понятие кривизны меры, которое - увы! - не работает в высших размерностях.

Из приведенных результатов видно, что множество точек, в которых главные значения не существуют, в определенном смысле "мало". Теорема 8.2 позволяет подтвердить гипотезу Вердеры, приведенную в [106; с. 203].

ПРеДЛОЖЕНИЕ 8.3. Пусть $\nu$ - конечная комплексная мера Радона в $\mathbb{C}, u$ пусть $G$ - множество точек z в $\mathbb{C}$, в которых преобразование Коши $\mathscr{C} \nu(z)$ не имеет конечных главных значении. Тогда $\gamma(G)=0$ (через $\gamma$, как и ранее, обозначена аналитическая емкость множества).

ДоказАтельство. Рассуждая от противного, предположим, что существуют мера $\nu$ и компакт $F \subset G$, для которого $\gamma(F)>0$. Применяя неравенство Толсы $\gamma \leqslant C \gamma_{1,+}$, а также соотношения (4.8) и (4.10), получим, что существует положительная мера $\mu \in \Sigma_{1}$ с носителем на $F$, для которой оператор $\mathscr{C}_{\mu}$ ограничен в $L^{2}(\mu)$. По теореме 8.2 предел $\lim _{\varepsilon \rightarrow 0} \mathscr{C}_{\varepsilon} \nu(z)$ существует и конечен $\mu$-почти всюду в $F$. Это противоречит предположению о том, что преобразование Коши не имеет главных значений во всех точках из $F$. Предложение доказано.

Возникает вопрос: верно ли, что для любого множества $G$ нулевой аналитической емкости найдется конечная мера $\nu$ такая, что преобразование $\mathscr{C} \nu(z)$ не имеет конечных главных значений ни в одной точке $z \in G$ ?

Обратная задача (существование главных значений преобразований по “хорошим" мерам влечет $n$-спрямляемость) также изучалась в ряде работ. Первый результат был получен Маттилой [64] для случая $n=1, d=2$ : если конечная неотрицательная борелевская мера $\mu$ в $\mathbb{C}$ такова, что ее нижняя 1-плотность положительна и преобразование Коши $\mathscr{C} \mu(z)$ имеет конечные главные значения н-почти всюду в $\mathbb{C}$, то н является 1-спрямляемой. В доказательстве использовалось понятие касательных мер. В [42] этот результат распространен на более общий класс ядер в $\mathbb{C}$, являющихся конечными линейными комбинациями функций $z^{2 k-1} /|z|^{2 k}, k=1,2, \ldots$ В то же время имеются нечетные ядра в $\mathbb{C}$, для которых эта импликация неверна - см. [43]. Многомерное обобщение принадлежит Маттиле и Прейсу [72] при несколько более сильном предположении о конечности и нижней, и верхней $n$-плотностей.

Толса [114] получил следующий результат.

Tеорема 8.4. Пусть $E$ - множество в $\mathbb{R}^{d}$ с $H^{n}(E)<\infty$, и nусть $0<n \leqslant d$. Для $n$-спрямляемости множества Е необходимо и достаточно, чтобы предел $\lim _{\varepsilon \rightarrow 0} R_{\varepsilon}^{n}\left(H^{n} \mid E\right)(x)$ существовал для $H^{n}$-почти всех $x \in E$.

Как отмечено в [114], необходимость (т. е. что $n$-спрямляемость влечет существование главных значений) была известна ранее - см. указанный выше результат Маттилы и Прейса.

В случае $n=1, d=2$ условие существования главных значений можно ослабить, заменив его условием $\mathscr{C}^{\#}\left(H^{1} \mid E\right)(z)<\infty$ для $H^{1}$-почти всех $x \in E$. 
Это показал Толса [107], используя кривизну меры. При $n>1$ возможность такой замены является открытым вопросом - см. [114].

Теорема 8.4 (точнее, достаточность) была получена Толсой как следствие более общего результата, усиливающего теоремы из работ [64] и [72].

Теорема 8.5. Пусть конечная борелевская мера $\mu$, иелое число $n \in(0, d]$ и множество $E$ в $\mathbb{R}^{d}$ таковъ, что в каждой точке $x \in E$ верхняя $n$-nлотность $\Theta^{*}(x)$ положительна и конечна и при всех $x \in E$ существуют главные значения преобразования $R^{n} \mu(x)$. Тогда Е является $n$-спрямляемым.

Нетрудно видеть, что без условия $\Theta^{*}(x)>0$ теорема 8.5 неверна. Отметим, что метод, использованный Толсой [114], отличен от подхода предшествующих авторов. Основной этап доказательства состоит в получении точных оценок $L^{2}$-нормы преобразований Рисса на графиках липшицевых функций.

В работе Вольберга и Майбороды [75] условие о существовании главных значений заменено ограниченностью функции $S \mu(x)$ из (7.2).

Теорема 8.6. Пусть $\mu$ - конечная мера Радона в $\mathbb{R}^{d}$, а число $s \in(0, d] u$ множество $E \subset \mathbb{R}^{d}$ такови, что $0<\Theta^{*}(x)<\infty u S \mu(x)<\infty$ для любой точки $x \in E$. Тогда s целое и множество $Е$ является s-спрямляемым.

Утверждение, что $s$ целое, является следствием из теоремы 7.10. В доказательстве $s$-спрямляемости используются идеи работы [114], а также утверждение об ограниченности в $L^{2}(\mu)$ оператора $S: f \rightarrow S(f d \mu)$, представляющее и самостоятельный интерес.

8.3. Существование главных значений преобразования $R^{n} \mu$ и ограниченность оператора $R_{\mu}^{n}$. Начнем с несложного примера, показывающего, что из существования главных значений $\mu$-почти всюду не следует ограниченность оператора.

Пример 8.7. Существует 1-спрямляемое множество $\mathrm{E}$ в $\mathbb{C}$ такое, что $H^{1}(E)<\infty$, преобразование Коши $\mathscr{C}\left(H^{1} \mid E\right)$ имеет главные значения $H^{1}$-почmи всюду на $E$, но оператор Коши $\mathscr{C}_{H^{1} \mid \text { Е }}$ не ограничен в $L^{2}\left(H^{1} \mid E\right)$.

Конечно, в силу теоремы 8.4 существование главных значений $H^{1}$-почти всюду следует из 1-спрямляемости и наоборот, но в нашем примере наличие обоих этих свойств будет очевидным.

ДокАЗАТЕЛЬство. Пусть $N_{0}=0$ и $\left\{N_{k}\right\}_{k=1}^{\infty}-$ последовательность натуральных чисел, для которой $N_{k+1}-N_{k} \rightarrow \infty$ при $k \rightarrow \infty$. Под квадратом будем понимать границу соответствующего множества (т. е. объединение четырех отрезков). Начнем построение с единичного квадрата и сделаем $N_{1}-N_{0}$ шагов построения "углового" канторова множества $E^{1 / 4}$ (см. п. 6.6). Получим $4^{N_{1}-N_{0}}$ квадратов со сторонами $4^{-N_{1}}$. Выберем один (любой) из этих квадратов, обозначим его через $E_{1}$ и дальнейшее построение будем проводить только с ним. Оставшиеся $4^{N_{1}-N_{0}}-1$ квадратов образуют часть искомого множества $E$, для которой построение закончено. В выбранном квадрате $E_{1}$ сделаем еще $N_{2}-N_{1}$ шагов построения множества $E^{1 / 4}$, получив $4^{N_{2}-N_{1}}$ квадратов со 
сторонами $4^{-N_{2}}$. Опять продолжим построение лишь для одного из них, квадрата $E_{2}$, и т.д. Объединение всех построенных квадратов и будет искомым множеством $E$.

Действительно, 1-спрямляемость и существование главных значений $H^{1}$-почти всюду на $E$ очевидны. Легко видеть также, что $H^{1}(E)<\infty$. Осталось показать, что оператор $\mathscr{C}_{H^{1} \mid E}$ не ограничен. Положим для краткости $\mu=H^{1} \mid E$, $\mu_{k}=H^{1} \mid E_{k}$. Применим оператор $\mathscr{C}_{\mu}$ к характеристическим функциям $\chi_{E_{k}}$, $k=1,2, \ldots$. Имеем

$$
\left\|\mathscr{C}_{\mu}\left(\chi_{E_{k}}\right)\right\|_{L^{2}(\mu)}^{2}=\left\|\mathscr{C} \mu_{k}\right\|_{L^{2}(\mu)}^{2}>\left\|\mathscr{C} \mu_{k}\right\|_{L^{2}\left(\mu_{k}\right)}^{2} .
$$

Из (6.1) нетрудно вывести оценку $\left\|\mathscr{C} \mu_{k}\right\|_{L^{2}\left(\mu_{k}\right)}^{2} \geqslant c\left(N_{k+1}-N_{k}\right) 4^{-N_{k}}$ с абсолютной постоянной $c$. Отсюда следует соотношение $\left\|\mathscr{C}_{\mu}\right\|_{L^{2}(\mu)}^{2} \geqslant c\left(N_{k+1}-N_{k}\right) \rightarrow \infty$, что нам и требовалось.

Заметим, что построенное выше множество $E$ является примером 1-АД-регулярного, 1-спрямляемого, но не равномерно 1-спрямляемого множества. Подобная конструкция (без подробного обоснования) приведена в [29; с. 9].

Естественно возникает обратная задача: влечет ли $L^{2}$-ограниченность операторов существование $\mu$-почти всюду главных значений соответствующих преобразований? (Отметим, что при весьма широких предположениях относительно ядер $K$ существование предела

$$
\lim _{\varepsilon \rightarrow 0} \int_{|x-y|>\varepsilon} K(x, y) d \mu(y)
$$

$\mu$-почти всюду влечет существование предела

$$
\lim _{\varepsilon \rightarrow 0} \int_{|x-y|>\varepsilon} K(x, y) f(y) d \mu(y)
$$

$\mu$-почти всюду для любой $f \in L^{1}(\mu)$.)

В случае оператора Коши утвердительный ответ на поставленный вопрос следует из теоремы 8.2 , если взять $\nu=\mu$. Более того, основным этапом доказательства теоремы 8.2 в [106] явилось следующее утверждение: если мера $\mu$ конечна, то из ограниченности оператора Коши $\mathscr{C}_{\mu}$ в $L^{2}(\mu)$ следует существование предела $\lim _{\varepsilon \rightarrow 0} \mathscr{C}_{\varepsilon} \mu(z)$ для $\mu$-почти всех $z \in \mathbb{C}$.

Мы уже говорили, что справедливость многомерного аналога теоремы 8.2 является открытым вопросом. Сформулируем в виде гипотезы частный случай этого утверждения (см. также [73; с. 269]).

ГиПотезА 8.8. Пусть мера Радона $\mu$ в $\mathbb{R}^{d}$ такова, что при некотором $n \leqslant d$ оператор $R_{\mu}^{n}$ ограничен в $L^{2}(\mu)$. Тогда предел $\lim _{\varepsilon \rightarrow 0} R_{\varepsilon}^{n} \mu(x)$ существует и конечен н-почти всюду в $\mathbb{R}^{d}$.

Эта гипотеза интересна еще и тем, что для произвольных ядер КЗ соответствующее утверждение неверно. Давид [24] построил ядро КЗ размерности 1 в $\mathbb{R}^{2}$, для которого оператор $T$ ограничен в $L^{2}(\mu)$ с мерой $\mu$ на канторовом множестве $E^{1 / 4}$ (определение см. в п. 6.6). При этом главные значения не существуют $\mu$-почти всюду в $\mathbb{R}^{d}$. Другой пример несуществования главных значений при $L^{2}(\mu)$-ограниченности оператора построен Коусионисом [14]. Таким 
образом, если гипотеза 8.8 верна, то ядра Рисса обладают особенно хорошими свойствами.

В качестве компенсации недоказанности этого утверждения Маттила и Вердера [73] установили аналог гипотезы 8.8 для слабой сходимости срезанных операторов, причем в весьма общей постановке. Пусть $X$ - сепарабельное метрическое пространство с конечной борелевской мерой $\mu$, удовлетворяющей теореме Витали о покрытии. Пусть, далее, $K: X \times X \backslash\{(x, y): x=y\} \rightarrow \mathbb{R}-$ антисимметричное измеримое по Борелю ядро, ограниченное на множестве $\{(x, y) \in X \times X: \operatorname{dist}(x, y)>\delta\}$ при любом $\delta>0$. Максимальный оператор $T^{\#}$ и срезки $T_{\varepsilon}$ определяются так же, как в п. 3.1 .

Tеорема 8.9 [73; теорема 1.4]. Пусть оператор $T^{\#}$ ограничен в $L^{2}(\mu)$. Тогда срезанные операторы $T_{\varepsilon}$ слабо сходятся в $L^{2}(\mu)$ в том смысле, что существует ограниченный линейный оператор $T: L^{2}(\mu) \rightarrow L^{2}(\mu)$, для которого

$$
\lim _{\varepsilon \rightarrow 0} \int T_{\varepsilon}(f) g d \mu=\int T(f) g d \mu, \quad f, g \in L^{2}(\mu) .
$$

Кроме того, для $\mu$-почти всех $z \in X$ справедливо равенство

$$
T(f)(z)=\lim _{r \rightarrow 0} \frac{1}{\mu(B(z, r))} \int_{B(z, r)}\left(\int_{X \backslash B(z, r)} K(x, y) f(y) d \mu(y)\right) d \mu(x) .
$$

В [17] изучается следующий вопрос: можно ли отказаться от условия ограниченности оператора $T^{\#}$ в теореме 8.9? В [73] отмечено, что по теореме Банаха-Штейнгауза слабая сходимость влечет равномерную ограниченность срезанных операторов $T_{\varepsilon}$, которая для широкого класса ядер равносильна ограниченности максимального оператора (например, для ядер К3 - см. теорему 3.6). Поэтому для неограниченных операторов $T^{\#}$ теорема 8.9 неверна. Тем не менее в [17; теорема 1.7] показано, что для широкого класса ядер и мер условие ограниченности можно опустить, если утверждать существование предела в (8.1) не для всех функций $f, g \in L^{2}(\mu)$, а лишь для плотных в $L^{2}(\mu)$ подклассов: конечных линейных комбинаций характеристических функций параллелепипедов и шаров в $\mathbb{R}^{d}$. Эти и родственные им результаты обсуждаются также в [68].

\section{9. Ограниченность операторов Кальдерона-Зигмунда и спрямляемость. Задача Давида-Семмса}

Читатель уже мог убедиться, что фундаментальным свойством сингулярных интегральных операторов, возникающим практически во всех задачах излагаемой теории, является ограниченность. Замечательная книга [29] Давида и Семмса, которая (как это часто бывает с исследованиями на переднем крае науки) часто цитировалась, но была прочитана, видимо, очень немногими (шутка Питера Джонса: “эту книгу прочли 4 человека: авторы, я и еще кто-то один"), вся посвящена одному вопросу: как связана ограниченность канонических сингулярных интегралов (в частности, преобразований Pисса) в $L^{2}(\mu)$ 
с геометрическими свойствами меры $\mu$ ? Проблема эта поднималась и раньше в книгах [28], [21] или в [20], а также (не в столь общей постановке) и во многих статьях.

В поисках критерия ограниченности операторов с ядрами из "естественного" класса Давид и Семмс [28], [29] пришли к понятию равномерной спрямляемости (на примере 8.7 мы видели, что просто спрямляемости недостаточно). Следуя [28], [29], введем класс $\mathscr{H}_{n}\left(\mathbb{R}^{d}\right)$ гладких нечетных функций $K: \mathbb{R}^{d} \backslash\{0\} \rightarrow \mathbb{R}$ таких, что

$$
|x|^{n+j}\left|\nabla^{j} K(x)\right| \in L^{\infty}\left(\mathbb{R}^{d} \backslash\{0\}\right), \quad j=0,1,2, \ldots .
$$

Важным примером функций из $\mathscr{H}_{n}\left(\mathbb{R}^{d}\right)$ являются компоненты $x_{i} /|x|^{n+1}, 1 \leqslant$ $i \leqslant d$, ядра Рисса $x /|x|^{n+1}$.

Теорема 9.1 [28], [29]. Пусть Е есть $n$-АД-регулярное множество в $\mathbb{R}^{d} u$ $\mu=H^{n} \mid$ E. Oператор $T=\int K(x-y) f(y) d \mu(y)$ ограничен в $L^{2}(\mu)$ для любого ядра $K \in \mathscr{H}_{n}\left(\mathbb{R}^{d}\right)$ тогда и толъко тогда, когда $E$ равномерно п-спрямляемо.

В [29; с. 49] показано, что теорема 9.1 справедлива и для более узкого, чем $\mathscr{H}_{n}\left(\mathbb{R}^{d}\right)$, класса ядер (разумеется, усиление здесь состоит в том, что ограниченность меньшего класса операторов все еще влечет равномерную $n$-спрямляемость). Маттила и Прейс [72; с. 493] заметили, что для этого достаточно взять еще более узкий класс ядер; метод работы [72], использующий касательные меры, отличен от подхода в [29].

Результаты Давида-Семмса получили дальнейшее развитие также в глубокой работе Толсы [115]. В ней вводится аналог $\beta$-чисел Джонса - $\alpha$-числа, в терминах которых получены, в частности, новые критерии равномерной спрямляемости мер, достаточные условия существования главных значений $\mu$-почти всюду, оценки $L^{2}(\mu)$-норм преобразования $T \mu$ и максимального преобразования $T^{\#} \mu$. Показано, что если мера $\mu$ равномерно спрямляема, то ограниченными в $L^{2}(\mu)$ будут все операторы с более широким, чем $\mathscr{H}_{n}\left(\mathbb{R}^{d}\right)$, классом нечетных ядер. А именно, достаточно потребовать выполнения (9.1) лишь для $j=0,1,2$.

В [29] обсуждается следующий вопрос: не будет ли достаточной для равномерной $n$-спрямляемости ограниченность лишь оператора $R_{\mu}^{n}$ с ядром Рисса (которая в силу теоремы 9.1 влекла бы ограниченность всех операторов с ядрами из класса $\mathscr{H}_{n}\left(\mathbb{R}^{d}\right)$ и его расширения, отмеченного Толсой)?

ГиПотеЗА 9.2. Пусть $E$ есть $n$-АД-регулярное множество в $\mathbb{R}^{d} u \mu=$ $H^{n} \mid E$. Оператор $R_{\mu}^{n}$ (m.е. набор из d операторов с ядрами $x_{i} /|x|^{n+1}$ ) ограничен в $L^{2}(\mu)$ тогда и только тогда, когда Е равномерно п-спрямляемо.

Согласно теореме 9.1, для доказательства гипотезы 9.2 нужно установить лишь одну ее часть: ограниченность оператора $R_{\mu}^{n}$ влечет за собой равномерную $n$-спрямляемость. Во время написания пионерских работ [22], [23], [28], [29] не был исследован даже случай оператора Коши по плоской мере. Введение Мельниковым понятия кривизны меры [79] в этот круг вопросов, находящихся на стыке гармонического анализа и геометрической теории меры, кардинально изменило ситуацию. Кроме того, как раз подоспели замечательные работы 
Джонса [50], [51] и Окикиолу [91]. Используя новое в то время понятие кривизны меры [79], Маттила, Мельников и Вердера [70] доказали гипотезу 9.2 в случае плоскости, что способствовало доказательству гипотезы Витушкина Давидом в 1998 г. (см. теорему 2.1).

ТЕОРема 9.3 [70]. Пусть Е - замкнутое 1-АД-регулярное подмножество комплексной плоскости. Справедливы следующие утверждения.

(a) Oператор Коши $\mathscr{C}_{H^{1} \mid \text { E }}$ ограничен в $L^{2}\left(H^{1} \mid E\right)$ тогда и только тогда, когда множество Е содержится в АД-регулярной кривой (и, значит, равномерно 1-спрямляемо - см. n. 8.1).

(b) Аналитическая емкость множества Е равна нулю тогда и только тогда, когда Е совершенно неспрямляемо.

Часть (b) уже обсуждалась в разделе 2. Таким образом, для 1-АД-регулярных плоских множеств задача полностью решена. В [15; теорема 1.3] часть (а) теоремы 9.3 распространена на операторы с ядрами $K_{n}(z)=x^{2 n-1} /|z|^{2 n}, z=$ $x+i y \in \mathbb{C} \backslash\{0\}$.

Главным связующим звеном между анализом и геометрией являлась кривизна меры. Совсем недавно Назаров, Толса и Вольберг [84] получили прорывный результат, не использующий это понятие, при $d \geqslant 2, n=d-1$. Этот случай, по-видимому, наиболее интересен для приложений. Уже говорилось (см. раздел 4$)$, что множества $E$, не несущие положительную меру с ограниченным оператором $R_{\mu}^{d-1}$, суть устранимые особенности для липшицевых гармонических функций. Кроме того, при $n=d-1$ имеется тесная связь между задачей Давида-Семмса и регулярностью решений эллиптических дифференциальных уравнений с частными производными - см. [41] и имеющиеся там ссылки.

ТеОрема 9.4 [84]. Пусть $\mu$ есть $(d-1)$-АД-регулярная мера в $\mathbb{R}^{d}$. Если оператор $R_{\mu}^{d-1}$ ограничен в $L^{2}(\mu)$, то носитель $E$ меры $\mu$ равномерно $(d-1)$ спрямляем.

Теорема 9.4 усиливает результат работы [41]. В трудном доказательстве теоремы 9.4 можно выделить два основных ингредиента. Первый, идущий от работ Толсы [114], [115], - это геометрическое свойство меры, состоящее в том, что не может быть много клеток, в которых носитель меры близок к $(d-1)$-плоскости, но при этом распределение меры существенно отлично от лебеговой меры на данной плоскости. Вторая составляющая - подход из работ [35], [49], описанный в разделе 7. Этот подход позволил использовать идеи Матео и Толсы [59] и “превратить шкалы с низкими плотностями меры, которые были главным врагом в большинстве предыдущих подходов (например, при работе с касательными мерами. - Aвm.), в полезных друзей” (цитата из [84; с. 3]). Применяя упомянутые выше методы, Назаров, Толса и Вольберг выводят из условий теоремы 9.4 один из эквивалентных признаков равномерной спрямляемости, установленных Давидом и Семмсом [29], откуда и следует нужное утверждение. Но причина ограничения на $s$, описанная в п. 7.1 (отсутствие принципа максимума), и здесь препятствует распространению теоремы 9.4 на коразмерности, большие 1.

Рассмотрим более общую задачу, в которой не предполагается априори регулярность множества или меры. Разумеется, без АД-регулярности мы не можем 
ожидать равномерной спрямляемости: достаточно взять любое компактное не АД-регулярное множество положительной длины на прямой и $n=1, d=2$. Но будет ли иметь место просто спрямляемость?

ГиПотезА 9.5. Пусть $H^{n}(E)<\infty$ при некотором $n=1, \ldots, d-1$, и пусть $\mu=H^{n} \mid$ E. Если оператор $R_{\mu}^{n}$ ограничен в $L^{2}(\mu)$, то множество $E$ является n-спрямляемым.

Отметим, что хотя регулярность множества не предполагается, но оценка сверху $H^{n}(B(x, r) \cap E) \leqslant C r^{n}$, входящая в определение АД-регулярности, здесь является необходимой. Она следует из условия ограниченности оператора $R_{\mu}^{n}$ (см., например, $[21$; предложение 1.4 , с. 56]). Поэтому размерность множества $E$ не может быть меньше порядка $n$ сингулярности ядра. Если же размерность больше $n$, то оператор $R_{\mu}^{n}$ заведомо ограничен. Таким образом, содержательным будет только тот случай, когда эти величины равны. Напомним, что если гипотеза 7.1 верна, то ограниченность оператора $R_{\mu}^{n}$ возможна лишь при целых $s$.

В случае $d=2, n=1$ гипотезу 9.5 доказали Давид и Леже [54] - см. теорему 2.4 (мы уже отмечали, что ограниченность кривизны меры можно заменить ограниченностью преобразования Коши). Недавно замечательное продвижение было получено Назаровым, Толсой и Вольбергом [85], доказавшими эту гипотезу при $d \geqslant 2, n=d-1$.

Tеорема 9.6 [85]. Пусть множество $E \subset \mathbb{R}^{d}$ таково, что $H^{d-1}(E)<\infty$, и $\mu=H^{d-1} \mid E$. Eсли оператор $R_{\mu}^{d-1}$ ограничен в $L^{2}(\mu)$, то множество $E$ является $(d-1)$-спрямляемым.

Теорема 9.6 содержит теорему 2.4 как частный случай $d=2$. Заметим, что мера $\mu$ здесь уже не обязательно однородна. Доказательство теоремы 9.6 существенно отлично от аргументов из [54]. Оно развивает подход Пажо [92] и основано на теоремах 7.2, 9.4 и на следующей лемме.

Лемма 9.7 [85]. Пусть $\mu$ - конечная мера Радона с компактным носителем $в \mathbb{R}^{d}$, для которой $\mu(B(x, r)) \leqslant c r^{n}$ при всех $x \in \mathbb{R}^{d}, r>0$, и нижняя $n$-плотность $\Theta_{*}(x)$ положительна для $\mu$-почти всех $x \in \mathbb{R}^{d}$. Предположим, далее, что оператор $R_{\mu}^{n}$ ограничен в $L^{2}(\mu)$. Тогда найдутся конечные меры Радона $\mu_{k}, k \geqslant 1$, такие, что

(a) $\mu \leqslant \sum_{k \geqslant 1} \mu_{k}$;

(b) меры $\mu_{k}, k \geqslant 1$, являются $n$-АД-регулярными (с константами, зависящими от $k$ );

(c) операторъ $R_{\mu_{k}}^{n}$ ограниченъ в $L^{2}(\mu)$.

ДокАЗАТЕЛЬСТво теоремы 9.6. Можно считать множество $Е$ ограниченным. Положим $\mu=H^{d-1}\left|E, E_{0}:=\left\{x \in E: \Theta_{*}(x)=0\right\}, \mu_{0}=\mu\right| E_{0}$. Тогда для плотностей меры $\mu_{0}$ имеем

$$
0=\Theta_{*}\left(x, \mu_{0}\right) \leqslant \Theta^{*}\left(x, \mu_{0}\right) \leqslant C \quad \text { для } \mu_{0} \text {-почти всех } x \in \mathbb{R}^{d} .
$$

Кроме того, оператор $R_{\mu_{0}}^{d-1}$ ограничен в $L^{2}\left(\mu_{0}\right)$. По теореме 7.2 , справедливой при $s=d-1$, имеем $\mu_{0}=0$. Следовательно, $\Theta_{*}(x, \mu)>0$ для $\mu$-почти всех 
$x \in \mathbb{R}^{d}$. Пусть $\mu_{k}-$ меры из леммы 9.7. K ним применима теорема 9.4 , согласно которой носители мер $\mu_{k}$ являются $(d-1)$-спрямляемыми. Значит, множество $F:=\bigcup_{k} \operatorname{supp} \mu_{k}$ также $(d-1)$-спрямляемо. Поскольку

$$
H^{d-1}(E \backslash F)=\mu\left(\mathbb{R}^{d} \backslash F\right) \leqslant \sum_{k} \mu_{k}\left(\mathbb{R}^{d} \backslash F\right)=0
$$

то и множество $E$ является $(d-1)$-спрямляемым. Теорема доказана.

В разделе 4 мы видели, что если компакт $E \subset \mathbb{R}^{d}$ не устраним для липшицевых гармонических функций, то он несет положительную меру $\mu$, для которой оператор $R_{\mu}^{d-1}$ ограничен в $L^{2}(\mu)$. Из этого результата Вольберга [126] и теоремы 9.4 нетрудно вывести еще одно важное следствие [85; раздел 4]: если компакт $E \subset \mathbb{R}^{d}$ совершенно $(d-1)$-неспрямляем (т. е. не содержит $(d-1)$-спрямляемых подмножеств положительной $H^{d-1}$-меры) и $H^{d-1}(E)<\infty$, то $E$ устраним для липшицевых гармонических функций, т. е. $\kappa(E)=0$. Обратная импликация была доказана Маттилой и Парамоновым [71]. Получается следующий многомерный аналог теоремы 2.1, подтверждающей гипотезу Витушкина.

Tеорема 9.8 [85]. Компакт $E \subset \mathbb{R}^{d}$ с $H^{d-1}(E)<\infty$ устраним для липиицевых гармонических функиии в $\mathbb{R}^{d}$ тогда и только тогда, когда $E$ совершенно $(d-1)$-неспрямляем.

Подчеркнем еще раз, что теоремы 9.4, 9.6 и 9.8 доказаны только в случае множеств коразмерности 1. В случае бо́льших коразмерностей вопрос открыт. Надежда заполнить этот пробел связана с верой в справедливость, если не вообще, то хотя бы в справедливость принципа максимума из раздела 7 - см. гипотезу 7.3.

Пусть теперь $H^{d-1}(E)=\infty$. Если $d=2, n=1$, то гипотеза Витушкина неверна - см. раздел 2. Более того, в [71; следствие 3.3] показано, что и в многомерном случае естественный аналог длины Фавара несоизмерим с емкостью $\kappa$, а значит, и с $\gamma_{d-1}$ (см. (4.3), (4.6)). Но в случае $d=2$ известно описание устранимых особенностей (множеств аналитической емкости нуль) хотя бы в терминах кривизны меры, имеющих геометрический характер (теорема 2.3 Толсы). При $d>2$ нет даже такого описания. "Грубое отсутствие" кривизны меры в высших размерностях пока остается невосполнимым.

\section{Заключение}

Данный обзор не претендует на полноту. В нем освещены далеко не все результаты, относящиеся к изучаемой теории. Например, мы не излагаем такой важный раздел, как двухвесовые оценки операторных норм. Эти результаты являются непосредственным развитием изложенной здесь теории и заслуживают отдельной статьи. Тем не менее мы надеемся, что эта работа дает представление как о современном состоянии рассматриваемых вопросов, так и о применяемых методах и привлечет внимание к ряду интереснейших задач, сформулированных в работе. 
Приносим искреннюю благодарность профессору Федору Львовичу Назарову за внимание к работе, ее обсуждение и ряд ценных комментариев, способствовавших ее улучшению. Мы благодарны также рецензенту за полезные замечания.

\section{Список литературы}

[1] D. R. Adams, V. Ya. Eiderman, "Singular operators with antisymmetric kernels, related capacities, and Wolff potentials", Int. Math. Res. Not. IMRN, 2012:24 (2012), $5554-5584$.

[2] D. R. Adams, L. I. Hedberg, Function spaces and potential theory, Grundlehren Math. Wiss., 314, Springer-Verlag, Berlin, 1996, xii+366 pp.

[3] L. V. Ahlfors, "Bounded analytic functions", Duke Math. J., 14:1 (1947), 1-11.

[4] Дж. М. Андерсон, В.Я. Эйдерман, “Оценки преобразования Коши точечных масс (логарифмической производной многочлена)", Докл. РАН, 401:5 (2005), 583-586; англ. пер.: J. M. Anderson, V. Ya. Ė̌̀derman, "Estimates for the Cauchy transform of point masses (the logarithmic derivative of polynomials)", Dokl. Math., 71:2 (2005), 241-244.

[5] J. M. Anderson, V. Ya. Eiderman, "Cauchy transforms of point masses: the logarithmic derivative of the polynomial", Ann. of Math. (2), 163:3 (2006), 1057-1076.

[6] M. Bateman, A. Volberg, "An estimate from below for the Buffon needle probability of the four-corner Cantor set", Math. Res. Lett., 17:5 (2010), 959-967; arXiv: 0807.2953.

[7] M. Bond, I. Laba, A. Volberg, "Buffon's needle estimates for rational product Cantor sets", Amer. J. Math. (to appear); arXiv: 1109.1031.

[8] M. Bond, A. Volberg, "Buffon needle lands in $\epsilon$-neighborhood of a 1-dimensional Sierpinski gasket with probability at most $|\log \epsilon|^{-c} "$, C. R. Math. Acad. Sci. Paris, 348:11-12 (2010), 653-656.

[9] M. Bond, A. Volberg, "Buffon's needle landing near Besicovitch irregular self-similar sets", Indiana Univ. Math. J. (to appear); arXiv: 0912.5111.

[10] A. P. Calderón, "Cauchy integrals on Lipschitz curves and related operators", Proc. Nat. Acad. Sci. U.S.A., 74:4 (1977), 1324-1327.

[11] A.P. Calderón, "Commutators, singular integrals on Lipschitz curves and applications", Proceedings of the International congress of mathematicians (Helsinki, 1978), Acad. Sci. Fennica, Helsinki, 1980, 85-96.

[12] A.P. Calderón, A. Zygmund, "On the existence of certain singular integrals", Acta Math., 88:1 (1952), 85-139.

[13] Л. Карлесон, Избранные проблемы теории исключительных множеств, Мир, M., 1971, 126 с.; пер. с англ.: L. Carleson, Selected problems on exceptional sets, Van Nostrand Math. Studies, 13, D. Van Nostrand Co., Inc., Princeton, NJ-Toronto, ON-London, 1967, v+151 pp.

[14] V. Chousionis, "Singular integrals on Sierpinski gaskets", Publ. Mat., 53:1 (2009), 245-256.

[15] V. Chousionis, J. Mateu, L. Prat, X. Tolsa, "Calderón-Zygmund kernels and rectifiability in the plane", Adv. Math., 231:1 (2012), 535-568.

[16] V. Chousionis, J. Mateu, L. Prat, X. Tolsa, "Capacities associated with Calderón-Zygmund kernels", Potential Anal., 38:3 (2013), 913-949; arXiv: 1112.3849 .

[17] V. Chousionis, P. Mattila, "Boundedness and convergence for singular integrals of measures separated by Lipschitz graphs", Bull. Lond. Math. Soc., 42:1 (2010), 109-118. 
[18] M. Christ, "A $T(b)$ theorem with remarks on analytic capacity and the Cauchy integral", Colloq. Math., 60/61:2 (1990), 601-628.

[19] R. R. Coifman, A. McIntosh, Y. Meyer, "L'intégrale de Cauchy définit un opérateur borné sur $L^{2}$ pour les courbes lipschitziennes", Ann. of Math. (2), 116:2 (1982), 361-387.

[20] G. David, "Rectifiabilité quantifié et le problème du voyageur de commerce", Séminaire sur les Équations aux Dérivées Partielles, 1990-1991, Exp. № XIV, École Polytech., Palaiseau, 1991, 10 pp.

[21] G. David, Wavelets and singular integrals on curves and surfaces, Lecture Notes in Math., 1465, Springer-Verlag, Berlin, 1991, x+107 pp.

[22] G. David, "Unrectifiable 1-sets have vanishing analytic capacity", Rev. Mat. Iberoam., 14:2 (1998), 369-479.

[23] G. David, "Analytic capacity, Calderón-Zygmund operators, and rectifiability", Publ. Mat., 43:1 (1999), 3-25.

[24] G. David, "Des intégrales singulières bornées sur un ensemble de Cantor", $C$. $R$. Acad. Sci. Paris Sér. I Math., 332:5 (2001), 391-396.

[25] G. David, J. L. Journé, "A boundedness criterion for generalized Calderón-Zygmund operators", Ann. of Math. (2), 120:2 (1984), 371-397.

[26] G. David, J. L. Journé, S. Semmes, "Opérateurs de Calderón-Zygmund, fonctions para-accrétives et interpolation", Rev. Mat. Iberoam., 1:4 (1985), 1-56.

[27] G. David, P. Mattila, "Removable sets for Lipschitz harmonic functions in the plane", Rev. Mat. Iberoam., 16:1 (2000), 137-215.

[28] G. David, S. Semmes, Singular integrals and rectifiable sets in $R^{n}$ : Beyond Lipschitz graphs, Astérisque, 193, Soc. Math. France, Paris, 1991, 152 pp.

[29] G. David, S. Semmes, Analysis of and on uniformly rectifiable sets, Math. Surveys Monogr., 38, Amer. Math. Soc., Providence, RI, 1993, xii+356 pp.

[30] J. J. Dudziak, Vitushkin's conjecture for removable sets, Universitext, Springer, New York, 2010, xii+331 pp.

[31] В. Я. Эйдерман, "Мера Хаусдорфа и емкость, ассоциированная с потенциалами Коши", Матем. заметки, 63:6 (1998), 923-934; англ. пер.: V. Ya. Ėiderman, "Hausdorff measure and capacity associated with Cauchy potentials", Math. Notes, 63:6 (1998), 813-822.

[32] В.Я. Эйдерман, "Оценки картановского типа для потенциала Коши", Докл. PAH, 407:5 (2006), 604-608; англ. пер.: V. Ya. Ėiderman, "Cartan-type estimates for the Cauchy potential", Dokl. Math., 73:2 (2006), 273-276.

[33] В. Я. Эйдерман, "Оценки картановского типа для потенциалов с ядром Коши и с действительными ядрами", Матем. сб., 198:8 (2007), 115-160; англ. пер.: V. Ya. Eiderman, "Cartan-type estimates for potentials with Cauchy kernels and real-valued kernels", Sb. Math., 198:8 (2007), 1175-1220.

[34] V. Eiderman, F. Nazarov, A. Volberg, "Vector-valued Riesz potentials: Cartan-type estimates and related capacities", Proc. Lond. Math. Soc. (3), 101:3 (2010), 727-758.

[35] V. Eiderman, F. Nazarov, A. Volberg, "The $s$-Riesz transform of an $s$-dimensional measure in $\mathbb{R}^{2}$ is unbounded for $1<s<2$ ", J. Anal. Math. (to appear); arXiv: 1109.2260.

[36] V. Eiderman, A. Volberg, " $L^{2}$-norm and estimates from below for Riesz transforms on Cantor sets", Indiana Univ. Math. J., 60:4 (2011), 1077-1112; arXiv: 1012.0941.

[37] K. Falkoner, The geometry of fractal sets, Cambridge Tracts in Math., 85, Cambridge Univ. Press, Cambridge, 1986, xiv+162 pp.

[38] H. M. Farag, "The Riesz kernels do not give rise to higher-dimensional analogues of the Menger-Melnikov curvature", Publ. Mat., 43:1 (1999), 251-260. 
[39] Г. Федерер, Геометрическая теория меры, Наука, М., 1987, 760 с.; пер. с англ.: H. Federer, Geometric measure theory, Grundlehren Math. Wiss., 153, Springer-Verlag, New York, 1969, xiv+676 pp.

[40] J. Garnett, Analytic capacity and measure, Lecture Notes in Math., 297, Springer-Verlag, Berlin-New York, 1972, iv+138 pp.

[41] S. Hofmann, J. M. Martell, S. Mayboroda, Uniform rectifiability and harmonic measure III: Riesz transform bounds imply uniform rectifiability of boundaries of 1-sided NTA domains, arXiv: 1207.1527.

[42] P. Huovinen, Singular integrals and rectifiability of measures in the plane, Dissertation, Ann. Acad. Sci. Fenn. Math. Diss., 109, University of Jyväskylä, Jyväskylä, 1997, 63 pp.

[43] P. Huovinen, "A nicely behaved singular integral on a purely unrectifiable set", Proc. Amer. Math. Soc., 129:11 (2001), 3345-3351.

[44] T. Hytönen, Suile Liu, Dachun Yang, Dongong Yang, "Boundedness of CalderónZygmund operators on non-homogeneous metric measure spaces", Canad. J. Math., 64:4 (2012), 892-923; arXiv: 1011.2937.

[45] T. Hytönen, H. Martikainen, "Non-homogeneous $T b$ theorem and random dyadic cubes on metric measure spaces", J. Geom. Anal., 22:4 (2012), 1071-1107; arXiv: 0911.4387.

[46] T. Hytönen, H. Martikainen, "On general local Tb theorems", Trans. Amer. Math. Soc., 364:9 (2012), 4819-4846; arXiv: 1011.0642.

[47] T. Hytönen, F. Nazarov, The local Tb-theorem with rough test functions, arXiv: 1206.0907.

[48] B. Jaye, F. Nazarov, Reflectionless measures for Calderón-Zygmund operators, arXiv: $1309.6661 \mathrm{v} 1$.

[49] B. Jaye, F. Nazarov, A. Volberg, "The fractional Riesz transform and an exponential potential", Алгебра и анализ, 24:6 (2012), 77-123; St. Petersburg Math. J., 24:6 (2013), 903-938; 2012, arXiv: 1204.2135.

[50] P.W. Jones, "Square functions, Cauchy integrals, analytic capacity, and harmonic measure", Harmonic analysis and partial differential equations (El Escorial, 1987), Lecture Notes in Math., 1384, Springer, Berlin, 1989, 24-68.

[51] P. W. Jones, "Rectifiable sets and the traveling salesman problem", Invent. Math., 102:1 (1990), 1-15.

[52] P. W. Jones, T. Murai, "Positive analytic capacity but zero Buffon needle probability", Pacific J. Math., 133:1 (1988), 99-114.

[53] С.Я. Хавинсон, "Соотношения двойственности в теории аналитической емкости", Алгебра и анализ, 15:1 (2003), 3-62; англ. пер.: S. Ya. Khavinson, "Duality relations in the theory of analytic capacity", St. Petersburg Math. J., 15:1 (2004), $1-40$.

[54] J. C. Léger, "Rectifiability and Menger curvature", Ann. of Math. (2), 149:3 (1999), 831-869.

[55] C. Liaw, S. Treil, "Regularizations of general singular integral operators", Rev. Mat. Iberoam., 29:1 (2013), 53-74; arXiv: 1010.6184v2.

[56] A. Mas, X. Tolsa, "Variation and oscillation for singular integrals with odd kernel on Lipschitz graphs", Proc. Lond. Math. Soc. (3), 105:1 (2012), 49-86.

[57] J. Mateu, L. Prat, J. Verdera, "The capacity associated to signed Riesz kernels, and Wolff potentials", J. Reine Angew. Math., 578 (2005), 201-223.

[58] J. Mateu, L. Prat, J. Verdera, "Capacities associated with scalar signed Riesz kernels, and analytic capacity", Indiana Univ. Math. J., 60:4 (2011), 1319-1362; arXiv: 1004.2170. 


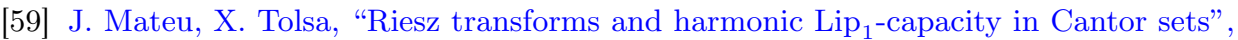
Proc. London Math. Soc. (3), 89:3 (2004), 676-696.

[60] J. Mateu, X. Tolsa, J. Verdera, "The planar Cantor sets of zero analytic capacity and the local T(b)-theorem", J. Amer. Math. Soc., 16:1 (2003), 19-28 (electronic).

[61] J. Mateu, X. Tolsa, J. Verdera, "On the semiadditivity of analytic capacity and planar Cantor sets", Harmonic analysis at Mount Holyoke (South Hadley, MA, 2001), Contemp. Math., 320, Amer. Math. Soc., Providence, RI, 2003, 259-278.

[62] P. Mattila, "Smooth maps, null-sets for integralgeometric measure and analytic capacity", Ann. of Math. (2), 123:2 (1986), 303-309.

[63] P. Mattila, Geometry of sets and measures in Euclidean spaces: Fractals and rectifiability, Cambridge Stud. Adv. Math., Cambridge Univ. Press, Cambridge, 1995, xii+343 pp.

[64] P. Mattila, "Cauchy singular integrals and rectifiability of measures in the plane", Adv. Math., 115:1 (1995), 1-34.

[65] P. Mattila, "Removability, geometric measure theory, and singular integrals", Potential theory - ICPT 94 (Kouty, 1994), de Gruyter, Berlin, 1996, 129-146.

[66] P. Mattila, "Search for geometric criteria for removable sets of bounded analytic functions", Cubo, 6:4 (2004), 113-132.

[67] P. Mattila, "Hausdorff dimension, projections, and the Fourier transform", Publ. Mat., 48:1 (2004), 3-48.

[68] P. Mattila, "Removability, singular integrals and rectifiability", Rev. Roumaine Math. Pures Appl., 54:5-6 (2009), 483-491.

[69] P. Mattila, M. S. Melnikov, "Existence and weak-type inequalities for Cauchy integrals of general measures on rectifiable curves and sets", Proc. Amer. Math. Soc., 120:1 (1994), 143-149.

[70] P. Mattila, M. S. Melnikov, J. Verdera, "The Cauchy integral, analytic capacity, and uniform rectifiability", Ann. of Math. (2), 144 (1996), 127-136.

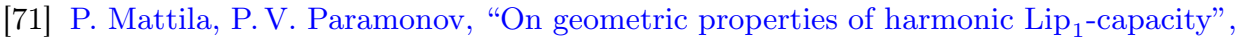
Pacific J. Math., 171:2 (1995), 469-491.

[72] P. Mattila, D. Preiss, "Rectifiable measures in $\mathbb{R}^{n}$ and existence of principal values for singular integrals", J. Lond. Math. Soc. (2), 52:3 (1995), 482-496.

[73] P. Mattila, J. Verdera, "Convergence of singular integrals with general measures", J. Eur. Math. Soc. (JEMS), 11:2 (2009), 257-271.

[74] S. Mayboroda, A. Volberg, "Finite square function implies integer dimension", C. $R$. Math. Acad. Sci. Paris, 347:21-22 (2009), 1271-1276.

[75] S. Mayboroda, A. Volberg, "Boundedness of the square function and rectifiability", C. R. Math. Acad. Sci. Paris, 347:17-18 (2009), 1051-1056.

[76] М.Я. Мазалов, П.В. Парамонов, К. Ю. Федоровский, "Условия $C^{m}$-приближаемости функций решениями эллиптических уравнений”, УМН, 67:6(408) (2012), 53-100; англ. пер.: M. Ya. Mazalov, P. V. Paramonov, K. Yu. Fedorovskiy, "Conditions for $C^{m}$-approximability of functions by solutions of elliptic equations", Russian Math. Surveys, 67:6 (2012), 1023-1068.

[77] В. Г. Мазья, В.П. Хавин, "Нелинейная теория потенциала”, УМН, 27:6(168) (1972), 67-138; англ. пер.: V. G. Maz'ya, V.P. Havin, "Non-linear potential theory", Russian Math. Surveys, 27:6 (1972), 71-148.

[78] В. Г. Мазья, В. П. Хавин, "Приложения $(p, l)$-емкости к нескольким задачам теории исключительных множеств", Матем. сб., 90(132):4 (1973), 558-591; англ. пер.: V. G. Maz'ya, V.P. Havin, "Use of $(p, l)$-capacity in problems of the theory of exceptional sets", Math. USSR-Sb., 19:4 (1973), 547-580.

[79] М. С. Мельников, "Аналитическая емкость: дискретный подход и кривизна меры”, Матем. сб., 186:6 (1995), 57-76; англ. пер.: M.S. Mel'nikov, "Analytic 
capacity: discrete approach and curvature of measure", Sb. Math., 186:6 (1995), 827-846.

[80] М. С. Мельников, "Сага о проблеме Пенлеве и аналитической емкости", Аналитические и геометрические вопросы комплексного анализа, Сборник статей. K 70-летию со дня рождения академика Анатолия Георгиевича Витушкина, Тр. МИАН, 235, Наука, М., 2001, 157-164; англ. пер.: M. S. Mel'nikov, "Saga of the Painlevé problem and analytic capacity", Proc. Steklov Inst. Math., 235 (2001), 150-157.

[81] M. Melnikov, J. Verdera, "A geometric proof of the $L^{2}$ boundedness of the Cauchy integral on Lipschitz graphs", Internat. Math. Res. Notices, 1995, № 7, 325-331.

[82] T. Murai, A real variable method for the Cauchy transform, and analytic capacity, Lecture Notes in Math., 1307, Springer-Verlag, Berlin, 1988, viii+133 pp.

[83] F. Nazarov, Y. Peres, A. Volberg, "The power law for the Buffon needle probability of the four-corner Cantor set”, Алгебра и анализ, 22:1 (2010), 82-97; St. Petersburg Math. J., 22:1 (2011), 61-72.

[84] F. Nazarov, X. Tolsa, A. Volberg, On the uniform rectifiability of AD regular measures with bounded Riesz transform operator: the case of codimension 1, arXiv: 1212.5229 .

[85] F. Nazarov, X. Tolsa, A. Volberg, "The Riesz transform, rectifiability, and removability for Lipschitz harmonic functions", Publ. Mat. (to appear); arXiv: 1212.5431.

[86] F. Nazarov, S. Treil, A. Volberg, "Cauchy integral and Calderón-Zygmund operators on nonhomogeneous spaces", Int. Math. Res. Not. IMRN, 1997, № 15, 703-726.

[87] F. Nazarov, S. Treil, A. Volberg, "Weak type estimates and Cotlar inequalities for Calderón-Zygmund operators on nonhomogeneous spaces", Int. Math. Res. Not. IMRN, 1998, № 9, 463-487.

[88] F. Nazarov, S. Treil, A. Volberg, "Accretive system Tb-theorems on nonhomogeneous spaces", Duke Math. J., 113:2 (2002), 259-312.

[89] F. Nazarov, S. Treil, A. Volberg, The Tb-theorem on non-homogeneous spaces that proves a conjecture of Vitushkin, Preprint CRM № 519, Barcelona, 2002, 85 pp.

[90] F. Nazarov, S. Treil, A. Volberg, "The Tb-theorem on non-homogeneous spaces", Acta Math., 190:2 (2003), 151-239.

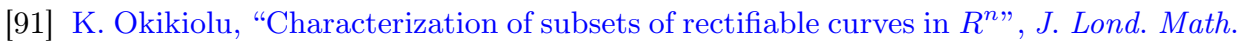
Soc. (2), 46:2 (1992), 336-348.

[92] H. Pajot, "Théorème de recouvrement par des ensembles Ahlfors-réguliers et capacité analytique", C. R. Acad. Sci. Paris Sér. I Math., 323:2 (1996), 133-135.

[93] H. Pajot, Analytic capacity, rectifiability, Menger curvature and the Cauchy integral, Lecture Notes in Math., 1799, Springer-Verlag, Berlin, 2002, xii+119 pp.

[94] П.В. Парамонов, "О гармонических аппроксимациях в $C^{1}$-норме", Матем. сб., 181:10 (1990), 1341-1365; англ. пер.: P. V. Paramonov, "On harmonic approximation in the $C^{1}$-norm", Math. USSR-Sb., 71:1 (1992), 183-207.

[95] Y. Peres, B. Solomyak, "How likely is Buffon's needle to fall near a planar Cantor set?", Pacific J. Math., 24:2 (2002), 473-496.

[96] L. Prat, "Potential theory of signed Riesz kernels: capacity and Hausdorff measure", Int. Math. Res. Not. IMRN, 2004:19 (2004), 937-981.

[97] L. Prat, "Principal values for the signed Riesz kernels of non-integer dimension", Rocky Mountain J. Math., 41:3 (2011), 869-883.

[98] L. Prat, "On the semiadditivity of the capacities associated with signed vector valued Riesz kernels", Trans. Amer. Math. Soc., 364:11 (2012), 5673-5691.

[99] A. Reznikov, A. Volberg, Cauchy independent measures and super-additivity of analytic capacity, arXiv: 1211.2675. 
[100] A. Ruiz de Villa, X. Tolsa, "Characterization and semiadditivity of the $\mathscr{C}^{1}$-harmonic capacity", Trans. Amer. Math. Soc., 362:7 (2010), 3641-3675.

[101] A. Ruiz de Villa, X. Tolsa, "Non existence of principal values of signed Riesz transforms of non integer dimension", Indiana Univ. Math. J., 59:1 (2010), 115-130; arXiv: 0812.2421.

[102] И. Стейн, Г. Вейс, Введение в гармонический анализ на евклидовых пространcтвах, Мир, М., 1974, 336 с.; пер. с англ.: Е. M. Stein, G. Weiss, Introduction to Fourier analysis on Euclidean spaces, Princeton Math. Ser., 32, Princeton Univ. Press, Princeton, NJ, 1971, x+297 pp.

[103] T. Tao, "A quantitative version of the Besicovitch projection theorem via multiscale analysis", Proc. Lond. Math. Soc. (3), 98:3 (2009), 559-584.

[104] E. Tchoundja, "Carleson measures for Hardy-Sobolev spaces", Complex Var. Elliptic Equ., 53:11 (2008), 1033-1046.

[105] E. Tchoundja, "Carleson measures for the generalized Bergman spaces via a T(1)-type theorem", Ark. Mat., 46:2 (2008), 377-406.

[106] X. Tolsa, "Cotlar's inequality without the doubling condition and existence of principal values for the Cauchy integral of measures", J. Reine Angew. Math., 502 (1998), 199-235.

[107] X. Tolsa, "Principal values for the Cauchy integral and rectifiability", Proc. Amer. Math. Soc., 128:7 (2000), 2111-2119.

[108] X. Tolsa, "A proof of the weak $(1,1)$ inequality for singular integrals with non doubling measures based on a Calderón-Zygmund decomposition", Publ. Mat., 45:1 (2001), 163-174.

[109] X. Tolsa, "On the analytic capacity $\gamma_{+}$", Indiana Univ. Math. J., 51:2 (2002), $317-343$.

[110] X. Tolsa, "Painlevé's problem and the semiadditivity of analytic capacity", Acta Math., 190:1 (2003), 105-149.

[111] X. Tolsa, "Finite curvature of arc length measure implies rectifiability: a new proof", Indiana Univ. Math. J., 54:4 (2005), 1075-1106.

[112] X. Tolsa, "Analytic capacity, rectifiability, and the Cauchy integral", International congress of mathematicians, v. 2, Eur. Math. Soc., Zürich, 2006, 1505-1527.

[113] X. Tolsa, "Painlevé's problem and analytic capacity", Collect. Math., Volume Extra Proceedings of the 7th International conference on harmonic analysis and partial differential equations held in El Escorial, June 21-25, 2004 (2006), 89-125.

[114] X. Tolsa, "Principal values for Riesz transforms and rectifiability", J. Funct. Anal., 254:7 (2008), 1811-1863.

[115] X. Tolsa, "Uniform rectifiability, Calderón-Zygmund operators with odd kernel, and quasiorthogonality", Proc. Lond. Math. Soc. (3), 98:2 (2009), 393-426.

[116] X. Tolsa, "Calderón-Zygmund capacities and Wolff potentials on Cantor sets", J. Geom. Anal., 21:1 (2011), 195-223; arXiv: 1001.2986.

[117] X. Tolsa, Analytic capacity, the Cauchy transform, and non-homogeneous Calderón-Zygmund theory, http://www.mat.uab.cat/ xtolsa/llibreweb.pdf; Progr. Math., 307, Birkhäuser/Springer, New York, 2014, 390 pp. (to appear).

[118] Nguyen Xuan Uy, "Removable sets of analytic functions satisfying a Lipschitz condition", Ark. Mat., 17:1-2 (1979), 19-27.

[119] Nguyen Xuan Uy, "An extremal problem on singular integrals", Amer. J. Math., 102:2 (1980), 279-290.

[120] J. Verdera, " $C^{m}$ approximation by solutions of elliptic equations, and CalderónZygmund operators", Duke Math. J., 55:1 (1987), 157-187.

[121] J. Verdera, "A weak type inequality for Cauchy transforms of finite measures", Publ. Mat., 36:2B (1992), 1029-1034. 
[122] J. Verdera, "The fall of the doubling condition in Calderón-Zygmund theory", Publ. Mat., Volume Extra - Proceedings of the 6th International conference on harmonic analysis and partial differential equations held in El Escorial, 3-7 July, 2000 (2002), 275-292.

[123] Дж. Вердера, М. С. Мельников, П. В. Парамонов, " $C^{1}$-аппроксимация и продолжение субгармонических функций”, Матем. сб., 192:4 (2001), 37-58; англ. пер.: J. Verdera, M. S. Mel'nikov, P. V. Paramonov, " $C^{1}$-approximation and extension of subharmonic functions", Sb. Math., 192:4 (2001), 515-535.

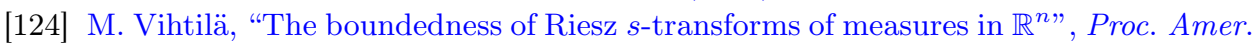
Math. Soc., 124:12 (1996), 3797-3804.

[125] А. Г. Витушкин, "Аналитическая емкость множеств в задачах теории приближений", УМH, 22:6(138) (1967), 141-199; англ. пер.: A. G. Vitushkin, "The analytic capacity of sets in problems of approximation theory", Russian Math. Surveys, 22:6 (1967), 139-200.

[126] A. Volberg, Calderón-Zygmund capacities and operators on nonhomogeneous spaces, CBMS Reg. Conf. Ser. Math., 100, Amer. Math. Soc., Providence, RI, 2003, iv +167 pp.

[127] А. Л. Вольберг, С. В. Конягин, "О мерах с условием удвоения", Изв. АН СССР. Cер. матем., 51:3 (1987), 666-675; англ. пер.: A. L. Vol'berg, S. V. Konyagin, "On measures with the doubling condition", Math. USSR-Izv., 30:3 (1988), 629-638.

[128] A. Volberg, B. D. Wick, "Bergman-type singular integral operators and the characterization of Carleson measures for Besov-Sobolev spaces on the complex ball", Amer. J. Math., 134:4 (2012), 949-992; arXiv: 0910.1142.

\section{Александр Львович Вольберг}

(Alexander L. Vol'berg)

Поступила в редакцию

Michigan State University, East Lansing, MI, USA

03.05.2013

E-mail: volberg@math.msu.edu

\section{Владимир Яковлевич Эйдерман} (Vladimir Ya. Ėiderman)

University of Wisconsin-Madison, Madison, WI, USA

E-mail: eiderman@math.wisc.edu 